\title{
Heterogeneous multiscale Monte Carlo models for radiation therapy using gold nanoparticles
}

\author{
by \\ Martin P. Martinov \\ A thesis submitted to the Faculty of Graduate and Postdoctoral Affairs \\ in partial fulfilment of the requirements for the degree of \\ Doctor of Philosophy \\ in
}

Physics

Specialization in Medical Physics

Ottawa-Carleton Institute for Physics

Department of Physics

Carleton University

Ottawa, Ontario, Canada

2019

(c) 2019 Martin P. Martinov 



\section{Abstract}

Gold NanoParticle (GNP) dose-enhanced radiation Therapy (GNPT) is a proposed radiotherapy approach which aims to improve dose localization. In complement to experimental techniques, Monte Carlo (MC) radiation transport simulations are used for GNPT dosimetry. Simulations on a tumour scale require complex geometry models that are reliable and efficient, two traits often in contention. This work introduces a general MC framework, the Heterogeneous MultiScale (HetMS) model, towards efficient and accurate GNPT simulation. The HetMS model combines distinct geometries of varying detail on different length scales into one simulation.

The HetMS framework is implemented in the fast MC code EGSnrc. EGSnrc, with custom applications and geometries, is cross-validated with PENELOPE and Geant4-DNA MC codes and is tested for self-consistency, passing the electron Fano cavity test. Simulations of microscopic scoring cavities containing GNPs across a cm-scale phantom were constructed using the HetMS method, enabling fast MC calculations of tissue dose on a tumour scale. Dose Enhancement Factors (DEFs), ratio of dose to tissue with GNPs over dose to tissue without, were determined at various tumour positions for many different GNPT scenarios.

A cell model, with nucleus and cytoplasm as two concentric spheres, containing GNPs is simulated. Less realistic but efficient modelling approaches of gold in the cell (e.g., a contiguous gold volume) are insufficient for realistic DEF calculations. Cell DEFs are sensitive to the distribution of GNPs within the cell, with highest DEFs for nucleus and cytoplasm when GNPs are distributed over the nuclear surface. By investigating variable cell/nucleus size and fluctuations in gold to a target cell and 12 neighbouring cells, expected variation in cell DEF is determined.

The above models are combined to create more detailed GNPT simulations. Cell DEFs are calculated at many positions within a tumour-sized volume, which, combined with the DEF variations computed previously provide a range of expected cell DEFs at each position 
within the tumour. These simulations provide many useful metrics towards GNPT; e.g., the lesser DEFs expected for a cluster of cells, the depth at which the primary fluence attenuation from gold drops DEF below unity, and the feasibility of different GNP configurations. 


\section{Acknowledgements}

I would like to thank Dr. Rowan Thomson, both as my supervisor during my graduate studies and as my employer before that. She has been an excellent guide for improving my investigative, writing, and presentation skills. I would not be the researcher I am today

without her. I would like to thank Dr. Dave Rogers and Dr. Frédéric Tessier for their assistance in understanding the more obscure aspects of the EGSnrc code. Finally, I would like to thank Dr. Devika Chithrani for getting me oriented in the field of GNPT and giving me a tour of her GNP laboratory.

I am grateful to my parents who have supported and loved me unconditionally from when I was a child all the way through my post-secondary and graduate studies. I am grateful to my roommate Matt, who has been understanding and supportive over my $\mathrm{PhD}$. Finally, I am grateful to Patty Oliver, Victor Malkov, and Nick Majtenyi, my PhD cohort, for making my time as a graduate student much more enjoyable.

I would like to acknowledge financial support from the Kiwanis Club of Ottawa Medical Foundation and Dr. Kanta Marwah Scholarship, the Natural Sciences and Engineering Research Council of Canada (NSERC), Canada Research Chairs (CRC) program, an Early Researcher Award from the Ministry of Research and Innovation of Ontario, and the Carleton University Research Office, as well as the computing resources from Compute/Calcul Canada and the Shared Hierarchical Academic Research Computing Network (SHARCNET). 


\section{Statement of originality}

This thesis is a summary of the author's research while enrolled as a graduate student at Carleton University. The majority of the content in this document has been published in peer-reviewed journals and/or presented at scientific conferences.

Dr. Rowan Thomson has contributed to all aspects of the research in this thesis. Dr. Thomson had a hand in guiding the direction of this work, provided assistance in editing all publications and presentations, and helped in aspects of calculation and analysis. Aside from the contributions of Dr. Thomson, all coding, computations, data analysis, and writing were carried out by the author of this thesis.

\section{Peer-reviewed papers}

1. Martinov, M. P., \& Thomson, R. M. "Heterogeneous multiscale Monte Carlo simulations for gold nanoparticle radiosensitization" Medical Physics, 44.2 (2017):644-653. Chapter 3

\section{Submitted papers}

1. Martinov, M. P., \& Thomson, R. M. "Technical Note: Taking EGSnrc to new lows: Development of egs++ lattice geometry and testing with microscopic geometries" Medical Physics, Dec. (2019) - Chapter 2

\section{Papers in preparation}

1. Martinov, M. P., \& Thomson, R. M. "Expected variation of dose-enhancement in GNP therapy I. Assessing single-cell dose enhancement" - Chapter 4 
2. Martinov, M. P., \& Thomson, R. M. "Expected variation of dose-enhancement in GNP therapy II. Computing cell dose enhancement expectation values using HetMS simulations of tumours" - Chapter 5 


\section{Conference abstracts}

(* indicates presenting author)

- Martinov*, M. P., \& Thomson, R. M. "Towards multiscale simulations with EGSnrc: tests on cellular length scales". Oral presentation, International Conference on Monte Carlo Techniques for Medical Applications, Montreal, Canada, May 16-21, 2019

- Martinov*, M. P., \& Thomson, R. M. "Pushing the limits of EGSnrc: Computing microscopic dose metrics on a macroscopic scale using multiscale modeling". Poster presentation, Council on Ionizing Radiation Measurements and Standards Annual Meeting, Gaithersburg, United States, April 16-18, 2018

- Martinov*, M. P., \& Thomson, R. M. "Abstract ID: 68 Heterogeneous multiscale simulations of radiation therapy with gold nanoparticles" Physica Medica: European Journal of Medical Physics, 42 (2017): 13-14. Oral presentation, International Conference on Monte Carlo Techniques for Medical Applications, Napoli, Italy, October $15-17,2017$.

- Martinov*, M. P., \& Thomson, R. M. "Heterogeneous multiscale simulations of gold nanoparticle radiotherapy considering intracellular nanoparticle distribution" Medical Physics, 44.8 (2017): 4392. Oral presentation, Canadian Organization of Medical Physicists 63rd Annual Scientific Meeting, Ottawa, Canada, July 12-15, 2017

- Martinov*, M. P., \& Thomson, R. M. "OC-0227: The heterogeneous multiscale model for efficient computation of microscopic dose metrics" Radiotherapy and Oncology, 123 (2017): S113. Oral presentation, European SocieTy for Radiotherapy \& Oncology, Vienna, Austria, May 5-9, 2017 
- Martinov*, M. P., \& Thomson, R. M. "SU-E-T-667: Radiosensitization Due to Gold Nanoparticles: A Monte Carlo Cellular Dosimetry Investigation of An Expansive Parameter Space" Medical Physics, 42.6 (2015): 3490. Poster presentation, American Association of Physicists in Medicine Annual Scientific Meeting, Anaheim, United States, July 31 - August 4, 2015 


\section{List of abbreviations and symbols}

DNA $\ldots \ldots \ldots$ DeoxyriboNucleic Acid

$\mathrm{H}_{2} \mathrm{O} \quad \ldots \ldots \ldots$ Water

$\mathbf{H}_{2} \mathbf{O}_{2} \ldots \ldots \ldots$ Hydrogen peroxide

GNP $\ldots \ldots \ldots$ Gold NanoParticles

GNPT …... Gold NanoParticle dose-enhanced radiation Therapy

MC .......... Monte Carlo

EGSnrc ...... Electron Gamma Shower MC code distributed by Canada's National Research Council

XCOM ...... Photon cross section database distributed by the United States' National Institute of Standards and Technology

ICRU ........ International Commission on Radiation Units and measurements

in vivo ....... From latin for 'within the living', describing scenarios in humans or animals

in vitro ...... From latin for 'in the glass', describing scenarios in labs (e.g., petri dishes)

Au $\ldots \ldots \ldots$ Atomic symbol for gold, often used as gold short-hand

DEF $\ldots \ldots \ldots$..... Dose Enhancement Factor

PEG $\ldots . . . \ldots$ PolyEthylene Glycol

CH $\ldots \ldots \ldots \ldots$ Condensed History

PRESTA … Parameter Reduced Electron-Step Transport Algorithm

$\mathbf{m g} / \mathbf{g} \ldots \ldots \ldots$ Gold concentration in milligrams of gold per gram of tissue

Geant4 ...... GEometry ANd Tracking MC code distributed by the European Organization for Nuclear Research

PENELOPE . Penetration and ENErgy LOss of Positrons and Electrons MC code distributed by the Nuclear Energy Agency

MDA-231 cells Human breast cancer cell line

TOPAS ..... An extension and wrapper to the Geant4 MC code

Geant4-DNA An extension to Geant4 focused on very low (below $1 \mathrm{keV}$ ) energy particle transport 
MCNP5 ..... Monte Carlo N-Particle code version 5 distributed by the Los Alamos National Laboratory

MCNP6 ...... Monte Carlo N-Particle code version 6 distributed by the Los Alamos National Laboratory

HetMS ..... Heterogeneous MultiScale (model/method)

egs_chamber . EGSnrc application used for efficient dose calculations

howfar () .... EGSnrc geometry method to get distance to next target

hownear () ... EGSnrc geometry method to get nearest boundary distance

is Where() . . E EGSnrc geometry method to get current region

$\left(r_{\text {cell }}, r_{\text {nuc }}\right) \ldots$ Combined shorthand of cell, nucleus radius

CPE ........ Charged Particle Equilibrium

MIRD ....... Medical Internal Radiation Dose

nDEF $\ldots \ldots$ nucleus Dose Enhancement Factor

cDEF ....... cytoplasm Dose Enhancement Factor

$\mathbf{n , c D E F} \ldots . . \quad$ nucleus and cytoplasm Dose Enhancement Factor

"peri" ...... GNPs configured in a shell about the nucleus (perinuclear region)

"1-endo" .... GNPs configured in a cellular compartment (endosome)

"4-endo" .... GNPs configured in four cellular compartments (endosomes)

$n_{\text {GNP }} \ldots \ldots \ldots$ Number of GNPs taken up by a single cell

$N_{\text {cell }} \ldots \ldots \ldots$ Number of cells per $\mathrm{cm}^{3}$ of tissue

$\% \Delta_{\text {DEF }}^{+} \ldots \ldots \quad$ expected percent upwards variation in cell DEF

$\% \Delta_{\text {DEF }}^{-} \cdots \cdots \quad$ expected percent downwards variation in cell DEF

$\% \Delta_{\text {DEF }} \ldots \ldots$. combined $\% \Delta_{\mathrm{DEF}}^{+}$and $\% \Delta_{\mathrm{DEF}}^{-}$variation 


\section{Contents}

1 Introduction $\quad 1$

1.1 Radiation in cancer therapy . . . . . . . . . . . . . . . . 1

1.2 Electron and photon interactions in radiotherapy . . . . . . . . . . . . 3

1.3 Gold nanoparticles for use in radiotherapy . . . . . . . . . . . . . . . 9

1.4 Biological aspects of GNPs in tissue . . . . . . . . . . . . . . . . . 10

1.5 MC simulations of radiation transport . . . . . . . . . . . . . . . 12

$1.6 \mathrm{MC}$ simulations of GNPT . . . . . . . . . . . . . . . . . . . 14

1.7 Thesis Purpose . . . . . . . . . . . . . . . . 17

2 EGSnrc simulations at low energies and short length scales 20

2.1 Introduction . . . . . . . . . . . . . . . . . 20

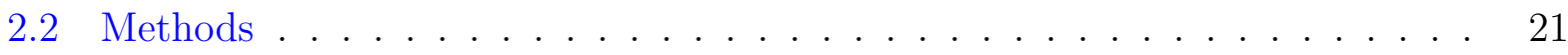

2.2.1 Lattice geometry implementation in EGSnrc . . . . . . . . . . . . 22

2.2.2 Cross-validation of EGSnrc . . . . . . . . . . . . . . . . 28

2.2.3 Electron Fano cavity test . . . . . . . . . . . . . . . . . . 31

2.3 Results . . . . . . . . . . . . . . . . . . . 33

2.3.1 Cross-validation . . . . . . . . . . . . . . . . 33

2.3.2 Electron Fano cavity test . . . . . . . . . . . . . . . . . 36

2.4 Discussion . . . . . . . . . . . . . . . . . . . . 37

2.5 Conclusion . . . . . . . . . . . . . . . . . . . 39

3 Tissue DEF in GNPT with HetMS 440

3.1 Introduction . . . . . . . . . . . . . . . . . . 40

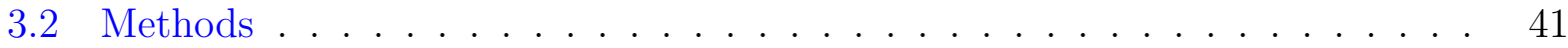


3.2.1 Heterogeneous multiscale modelling . . . . . . . . . . . . . . . 41

3.2.2 Parallel beam of monoenergetic photons incident on cylinder . . . . . 41

3.2.3 Brachytherapy source in a sphere with varying gold concentration . . 44

3.3 Results ............................... 46

3.3.1 Parallel beam of monoenergetic photons incident on cylinder . . . . . 46

3.3.2 Brachytherapy source in a sphere with varying gold concentration . . 51

3.4 Discussion . . . . . . . . . . . . . . . . . . . . . . 53

3.5 Conclusion . . . . . . . . . . . . . . . . . . 56

4 Single-cell DEF in GNPT $\quad 58$

4.1 Introduction . . . . . . . . . . . . . . . . . . . 58

4.2 Methods . . . . . . . . . . . . . . . . . . . 59

4.2.1 Modelling GNPs within a cell . . . . . . . . . . . . . . . 60

$4.2 .2 \mathrm{n}, \mathrm{cDEF}$ for variable cell size . . . . . . . . . . . . . . . 63

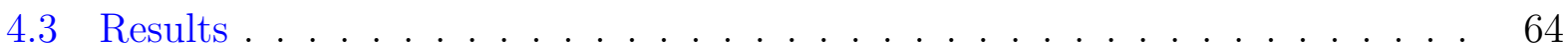

4.3.1 Modelling approach to GNPs within a cell . . . . . . . . . . . . 64

4.3.2 n,cDEF variation with GNP configuration and energy . . . . . . . 65

4.3.3 n,cDEF variation with GNP configuration and gold concentration . . 68

4.3.4 n,cDEF of cells with variable size . . . . . . . . . . . . . 70

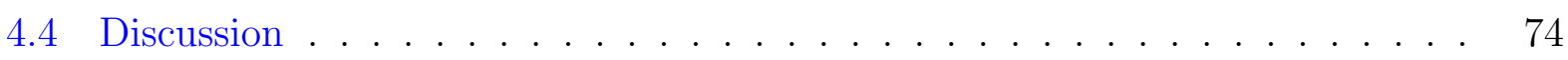

4.5 Conclusion . . . . . . . . . . . . . . . . . . . . 79

5 Cell DEF in GNPT with HetMS $\quad 80$

5.1 Introduction . . . . . . . . . . . . . . . . . 80

5.2 Methods . . . . . . . . . . . . . . . . . . . 81

5.2.1 Expected variation in cell DEF in GNPT . . . . . . . . . . . . . . 82

5.2.2 Microscopic scoring regions containing cells for HetMS . . . . . . . . 84

5.2.3 Tumour with constant macroscopic gold concentration . . . . . . . 85

5.2.4 Tumour with varying gold concentration . . . . . . . . . . 87

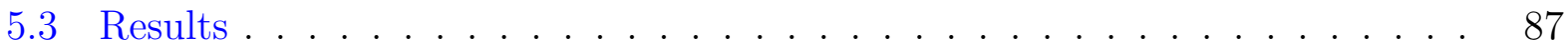

5.3.1 Expected variation in cell DEF in GNPT . . . . . . . . . . . . . . 87 
5.3.2 DEF in a tumour with constant macroscopic gold concentration . . . 92

5.3.3 DEF in a tumour with varying gold concentration in a brachytherapy scenario . . . . . . . . . . . . . . . . . 97

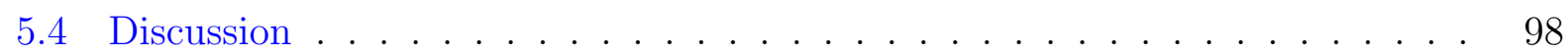

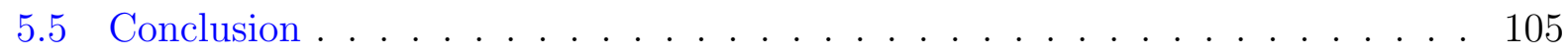

6 Conclusion $\quad 106$

6.1 EGSnrc simulations at short length scales . . . . . . . . . . . . . 108

6.2 Further applications of HetMS, in GNPT and beyond . . . . . . . . . . . 109

6.3 Considerations for GNPT . . . . . . . . . . . . . . . . 111

$\begin{array}{ll}\text { Appendices } & 113\end{array}$

$\begin{array}{ll}\text { A Cubic lattice implementation } & 113\end{array}$

A.1 closestPoint ()$\ldots \ldots \ldots \ldots \ldots \ldots \ldots$

A.2 is Where ()$\ldots \ldots \ldots \ldots \ldots \ldots$. . . . . . . . . . . . . . . . . . . . . . . . . . . . .

A.3 hownear ()$\ldots \ldots \ldots \ldots \ldots \ldots$

A.4 howfar ()$\ldots \ldots \ldots \ldots \ldots \ldots \ldots \ldots$

B Hexagonal lattice implementation $\quad 121$

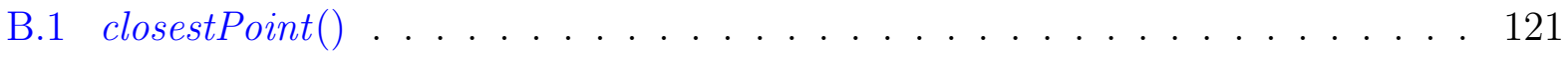

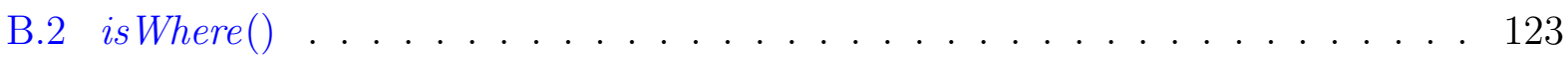

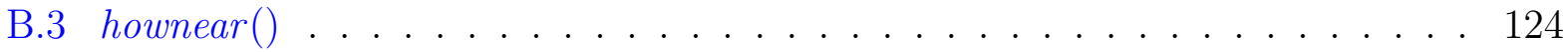

B.4 howfar ()$\ldots \ldots \ldots \ldots \ldots \ldots \ldots \ldots \ldots \ldots$

$\begin{array}{ll}\text { C Homogeneous to cellular DEF conversion factors } & 130\end{array}$

$\begin{array}{ll}\text { References } & 134\end{array}$ 


\section{List of Tables}

2.1 Homogeneous to discrete GNP conversion factors . . . . . . . . . . . . 35

2.2 Electron Fano cavity test . . . . . . . . . . . . . . . . . . . 36

3.1 HetMS efficiency values . . . . . . . . . . . . . . . . . . . . . . . 51

4.1 Linear fit coefficients for $\mathrm{n}, \mathrm{cDEF}$ over gold concentration . . . . . . . . . 68

$5.1 \% \Delta_{\mathrm{DEF}}$ variation in GNPT sample calculations $\ldots \ldots \ldots 9$

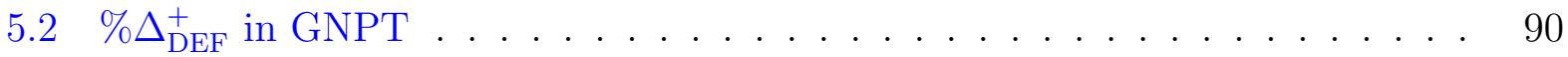

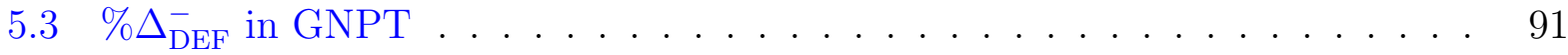

C.1 Homogeneous to $\mathrm{n}, \mathrm{cDEF}$ conversion factors at $20 \mathrm{mg} / \mathrm{g} \ldots \ldots \ldots \ldots$

C.2 Homogeneous to n,cDEF conversion factors at $10 \mathrm{mg} / \mathrm{g} \ldots \ldots \ldots$

C.3 Homogeneous to $\mathrm{n}, \mathrm{cDEF}$ conversion factors at $5 \mathrm{mg} / \mathrm{g} \ldots \ldots \ldots$ 


\section{List of Figures}

1.1 Photon interaction cross sections of water $\ldots \ldots \ldots 6$

1.2 Photon interaction cross sections of gold and tissue . . . . . . . . . . 8

2.1 Cubic lattice diagram . . . . . . . . . . . . . . . . . . . . . . . . . 24

2.2 Cubic lattice transport example . . . . . . . . . . . . . . . . . . 25

2.3 Hexagonal lattice diagram . . . . . . . . . . . . . . . . . . . 27

2.4 S-value calculation diagram $\ldots \ldots \ldots$

2.5 Homogeneous to discrete GNP conversion simulation diagram . . . . . . . 30

2.6 Electron Fano cavity test imagine . . . . . . . . . . . . . . . . . . . . . 32

2.7 MIRD S-values . . . . . . . . . . . . . . . . . . . . . . . . . . . . . 33

3.1 HetMS cylinder diagram . . . . . . . . . . . . . . . . . . . . . 43

3.2 HetMS sphere diagram and gold concentrations . . . . . . . . . . . . 45

3.3 DEF as a function of depth and energy . . . . . . . . . . . . . . . 47

3.4 DEF as a function of depth and GNP size . . . . . . . . . . . . . 49

3.5 DEF in volume with heterogeneous gold distribution . . . . . . . . . . . 52

4.1 Cell GNP configuration diagram . . . . . . . . . . . . . . . . . 60

4.2 Cell GNP model diagram . . . . . . . . . . . . . . . . . . . . 62

$4.3 \mathrm{n}, \mathrm{cDEF}$ vs energy for different gold models and configurations . . . . . . . 66

$4.4 \mathrm{n}, \mathrm{cDEF}$ vs energy for different concentrations and configurations . . . . . . 67

$4.5 \mathrm{n}, \mathrm{cDEF}$ vs gold concentration for different energies and configurations . . . . 69

$4.6 \mathrm{n}, \mathrm{cDEF}$ box and whisker plots of cell size statistics $\ldots \ldots \ldots . \ldots 72$

$4.7 \mathrm{n}, \mathrm{cDEF}$ vs energy for different cell sizes . . . . . . . . . . . . . 73 
5.113 cell cluster diagram . . . . . . . . . . . . . . . . . . 84

5.2 Cell microscopic scoring region image . . . . . . . . . . . . . . . . . 86

5.3 Spherical shell microscopic scoring region image . . . . . . . . . . . . 88

$5.4 \mathrm{n}, \mathrm{cDEF}$ as a function of depth and energy . . . . . . . . . . . . . . . . 94

$5.5 \mathrm{n}, \mathrm{cDEF}$ as a function of depth and gold concentration . . . . . . . . . . . 95

$5.6 \mathrm{n}, \mathrm{cDEF}$ over dispersed DEF ratio as a function of depth and energy . . . . . 96

$5.7 \mathrm{n}, \mathrm{cDEF}$ in volume after 33 days . . . . . . . . . . . . . . . . . . . . 99

$5.8 \mathrm{n}, \mathrm{cDEF}$ in volume after 5 and 59 days . . . . . . . . . . . . 100

5.9 Cell over dispersed DEF ratio in volume after 33 days . . . . . . . . . . . . . 101

5.10 Cell over dispersed DEF ratio in volume after 5 and 59 days . . . . . . . . . 102 


\section{Chapter 1}

\section{Introduction}

\subsection{Radiation in cancer therapy}

In the 1950s, Johns et $a l^{1-3}$ published an article introducing new radionuclide Cobalt-60 (emitting 1.2 and $1.3 \mathrm{MeV}$ photons) teletherapy treatment units ${ }^{4}$ installed in the University Hospital in Saskatchewan. These machines used ionizing radiation delivered to a cancer site as a form of clinical treatment. In the many decades following, the use of ionizing radiation to treat cancer has become more commonplace throughout cancer clinics internationally. Most clinics have moved on from the radionuclide based Cobalt-60 units to linear accelerators, particle accelerators that can generate electron currents (in the form of an electron pencil beam) with energies ranging from $4-35 \mathrm{MeV} .{ }^{5}$ The machines use a high- $\mathrm{Z}$ target (like Tungsten) in the path of the electron current to create a photon beam (spectra with mean energy $\sim 1 / 3$ of the electron current energy ${ }^{5}$ ) for treatment, or alternatively use the electron pencil beam (which can be spread to a wide beam using a scatter foil) directly on the patient. ${ }^{6}$

Beyond external photon (and electron) beam therapy, new forms of radiotherapy treatments have also been developed. Beams that use hadrons or ionized atoms instead of electrons have been developed due to lower beam scattering by the material it passes through and 
more localized energy deposition of hadrons (and atoms) near a focal point at a fixed penetration depth. ${ }^{7}$ Brachytherapy is another treatment that often uses relatively low-energy gamma emitting radionuclides $\left(\sim 30 \mathrm{keV}\right.$ for low dose-rate ${ }^{8}$ and $\sim 370 \mathrm{keV}$ for high doserate $^{9}$ ) embedded in small capsules (referred to as sources) that are inserted directly into the tumour either temporarily or permanently, typically for high-dose rate and low-dose rate treatments, respectively.

The mechanisms through which radiation can treat cancer fall into two categories, direct and indirect action of radiation interacting with a cell. ${ }^{10-12}$ For direct action, the radiation travelling through the cell interacts directly with a cell's molecular structure (such as DNA or mitochondria), damaging it. For indirect action, radiation interacts with the non-structural contents (mostly $\mathrm{H}_{2} \mathrm{O}$ ) of the cell and creates highly reactive free radicals. These free radicals in turn can react with molecular structures, leading to cell damage, or create compounds (such as $\mathrm{H}_{2} \mathrm{O}_{2}$ ) which can also be toxic to a cell. Through direct or indirect action, cell structure can be damaged enough to cause either necrotic (fatal) or clonogenic (inability to replicate) cell death. These are the mechanisms by which delivery of energy per unit mass, defined as dose (units of $\mathrm{Gy}=\mathrm{J} \cdot \mathrm{kg}^{-1}$ ), to a cell can kill it.

When delivering dose to a cancer tumour, treatments typically have two goals: (1) ensure dose to the tumour is sufficient to achieve tumour control (the extinction of clonogenic tumour cells at the end of treatment ${ }^{13}$ ) and (2) minimize the dose to healthy tissue, especially organs-at-risk. The former prevents the irradiated tumour cells from causing recurrence of the cancer, while the latter reduces health complications and the odds of developing secondary cancers. ${ }^{14}$ To better achieve these goals, new treatment methods are often proposed in the literature to improve on the dose localization (increase tumour dose, reduce dose to healthy tissue) to better achieve the aforementioned goals.

One proposed treatment suggests the use of Gold NanoParticles (GNPs) as an agent in photon beam cancer therapy, ${ }^{15}$ often referred to as GNP dose-enhanced radiation Therapy 
(GNPT). ${ }^{16}$ In GNPT, various delivery methods are used to fill a tumour with GNPs (while

minimizing GNP delivery to healthy tissue) and the differing radiological properties of gold are used to enhance dose to the tumour during a photon therapy treatment. Computational studies related to GNPT are the focus of this thesis, and this chapter provides the necessary background to describe computational simulations of GNPT. The physics of electrons and photons interacting with matter relevant to photon beam radiotherapy is described in section 1.2, where the differences in radiological properties of water and gold photon interactions are outlined. The synthesis of GNPs and their interactions on an organ and cellular scale are described in sections 1.3 and 1.4, respectively. Section 1.5 consists of a brief overview of Monte Carlo (MC) simulations of photon and electron transport; the section also describes EGSnrc, the MC code used in this thesis. Several MC studies (and their various approaches) in the field of GNPT are discussed in section 1.6. Section 1.7 describes the thesis purposes and provides an outline for the remainder of the thesis.

\subsection{Electron and photon interactions in radiotherapy}

Charged particles, such as electrons, travelling through a medium lose energy by means of Coulomb interactions with orbital electrons of the medium or by radiating photons through Coulomb interactions with the nuclei of the medium. In the case of large inelastic interactions with orbital electrons, the orbital electrons can receive enough kinetic energy to then also travel through the medium; such secondary electrons are referred to as knock-on or $\delta$ electrons. ${ }^{5}$ The frequency and likelihood of different interactions will vary with elemental composition of the medium through which electrons travel and the energy of the travelling electrons. The average energy loss per unit track length of the particle for a given energy is defined as the total stopping power, though often separated into radiative stopping power and collisional stopping power. Using a continuously slowing down approximation, the inverse total stopping can be integrated over the energy of an electron to provide a rough 
estimate of the average distance an electron of that energy would travel; although a useful estimate, it is not very realistic as the continuously slowing down approximation ignores the stochastic nature of electron travel and its multiple scattering.

Photons travelling through a medium can undergo different interactions depending on the energy of the photon. ${ }^{5}$ There are four (or five) more common interactions that are of interest for radiotherapy: photoelectric absorption, Rayleigh scatter, Compton scatter, or pair (and triplet) production. ${ }^{17}$ There is also the possibility of other types of photon interactions (e.g., Thomson scattering or photonuclear absorption), but these interactions are either relatively unlikely or only occur in very high energy linear accelerators (above $10 \mathrm{MV}$ ) which are less conventional in radiotherapy treatments, ${ }^{17}$ thus this section will focus on the more common interactions.

The photoelectric effect is the absorption of a photon by a bound electron. Photoelectric absorption produces an ejected electron with kinetic energy equal to that of the incoming photon minus the binding energy of the electron. The vacant orbital left by the ejected electron can lead to Auger electrons or x-ray fluorescence escaping the atom due to orbital electron relaxation.

Rayleigh scattering (also known as coherent scatter) is a photon interaction with a bound electron where only a very small amount of energy is transferred to the atom (not enough to free an electron) which results in a scattered photon with approximately the same initial energy. Rayleigh interactions scatter the incoming photon elastically by a small angle (relative to other scatter effects).

Compton scattering (also known as incoherent scatter) is the inelastic interaction of a photon with a loosely bound (binding energy is much less than incoming photon energy) electron. Compton interactions result in a scattered electron and photon. The scattered particles (and the ionized atom) conserve energy and momentum, with the particles scattering with variable kinetic energy depending primarily on scatter angle.

\subsection{ELECTRON AND PHOTON INTERACTIONS IN RADIOTHERAPY}


Photons with energies above the combined rest mass of an electron and a positron can undergo pair production or triplet production for energies above 2 combined electron and positron masses. ${ }^{5}$ Pair production is the transformation of a photon into an electron-positron pair in the Coulomb field of a nucleus. Pair production results in an electron-positron pair with total kinetic energy equal to the energy of the incoming photon minus the rest mass of the electron and positron (and a small amount of kinetic energy transferred to the nucleus). The fraction of kinetic energy imparted to the electron and positron depends on the scattering angle. An analogous process, triplet production, can also occur when a photon transforms into a electron-positron pair in the Coulomb field of an electron rather than a nucleus; in this case the kinetic energy transferred to the electron is significant and results in a scattered (not generated) electron in addition to the generated electron-positron pair.

The probability of a photon interacting with a material depends on both the energy of the photon and the elemental composition of the medium through which it travels, typically quantified as an interaction cross section. Figure 1.1 gives interaction cross sections of photons in water taken from the National Institute of Standards and Technology's XCOM database. ${ }^{18}$ Photoelectric absorption is the predominant interaction at low energies. Photon scatter (Compton and Rayleigh) start to dominate with increasing energy (0.1-1 MeV), depending on the material. Pair and triplet production only occur at energies above $1.022 \mathrm{MeV}$, where the production cross section increases quickly with energy and becomes the predominant interaction at high energies.

Beyond the cross section energy dependence shown in figure 1.1, the photon cross sections (macroscopic; units of $\mathrm{cm}^{2} / \mathrm{g}$ ) also have a significant dependence on the atomic number $(Z)$ of the material. Incoherent scatter cross sections scale linearly with $Z$, pair production and coherent scatter cross sections scale with $Z^{2}$ and the photoelectric cross sections scale with $Z^{4}$ (at low energies) to $Z^{4.6}$ (at high energies). ${ }^{19}$ The photoelectric cross section, especially, varies significantly with the $Z$ of the material. Figure 1.2 shows the relative con-

\subsection{ELECTRON AND PHOTON INTERACTIONS IN RADIOTHERAPY}




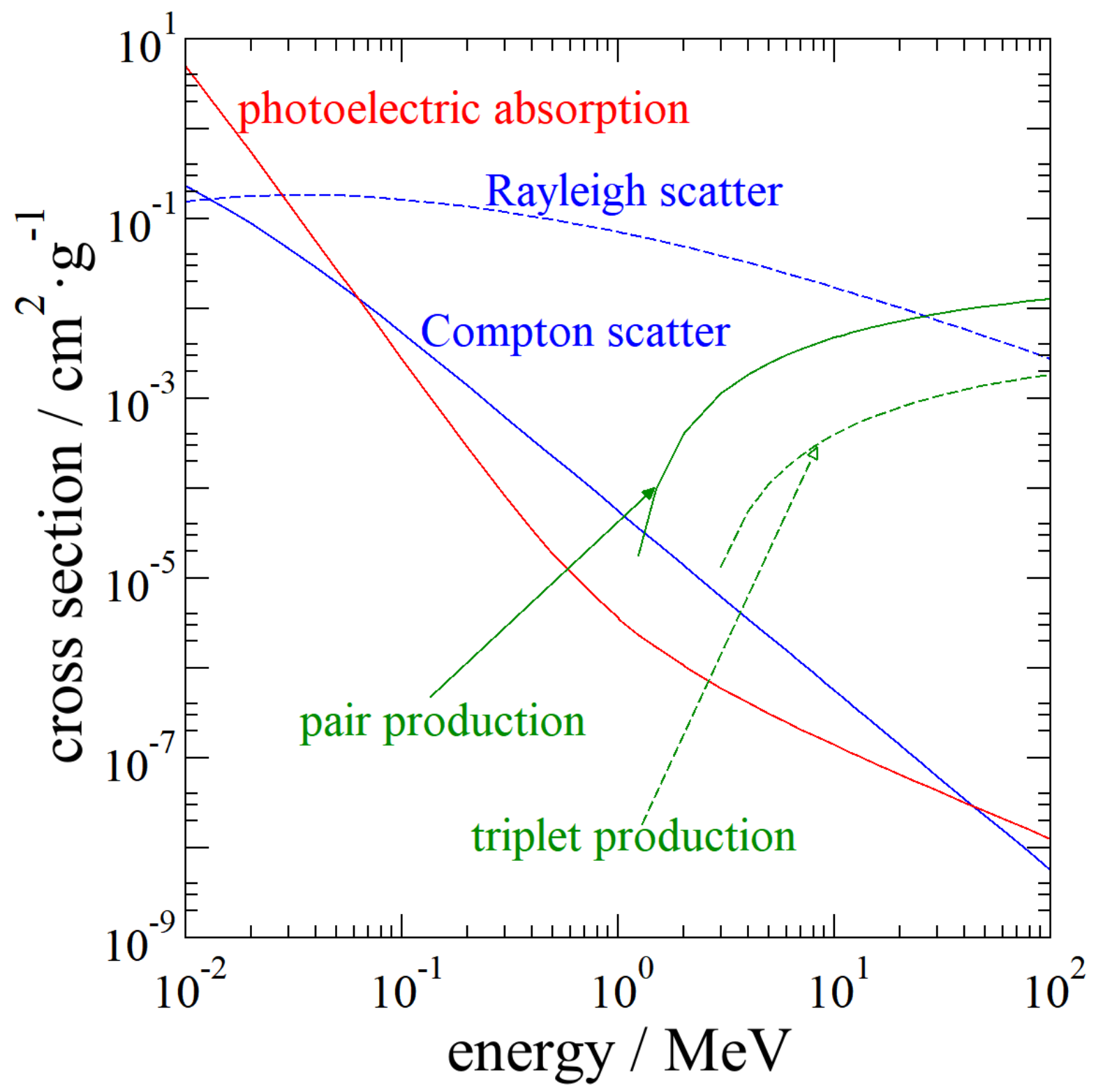

Figure 1.1: Plot of the cross sections all interaction processes of water taken from the National Institute of Standards and Technology's XCOM database. ${ }^{18}$ 
tribution of photoelectric absorption to the total cross section for gold and ICRU defined tissue. $^{20}$ Unlike in tissue, where the photoelectric effect is relatively unlikely at energies above $100 \mathrm{keV}$, photon interactions in gold are predominantly photoelectric at energies below $400 \mathrm{keV}$; in this low-energy range, the total interaction cross sections of gold are much larger than those of water.

In the context of radiotherapy, the dose is the primary quantity of interest and the primary contribution to dose is electronic inelastic collisions in the material. Thus, interactions that produce more energetic electrons (or positrons) are desired and interactions for which a photon scatters out of the target volume with some kinetic energy are unwanted. Both the photoelectric effect and pair production interactions result in scattered electrons (or positrons) with most of the incident photons' kinetic energy, minus electron binding energy or two electron rest masses, respectively. Conversely, Compton scattering imparts only some of the incident photon's kinetic energy to the electron and results in a scattered photon that could leave the target volume before interacting again. These reactions all have different fractions of incident photon energy transferred into scattered charged particle kinetic energy. In the context of photon interactions in a tumour, the largest energy transfer fraction possible is desired.

The goal in cancer treatment is to deposit as much dose as possible to the tumour and as a little as possible to the surrounding tissue. If for a radiotherapy treatment, gold were to be injected throughout the tumour volume, then photon interactions would be more likely to occur in the tumour (i.e., the tumour would have more electrons generated within it over the course of the treatment) at energies $<400 \mathrm{keV}$, as seen in figure 1.2 . The photoelectric effect would also be predominant for a higher range of energies, resulting in a higher fraction of incident photon energy transferred to electrons within the tumour. This is the motivation behind the use of GNPs distributed throughout a tumour to achieve a large Dose 


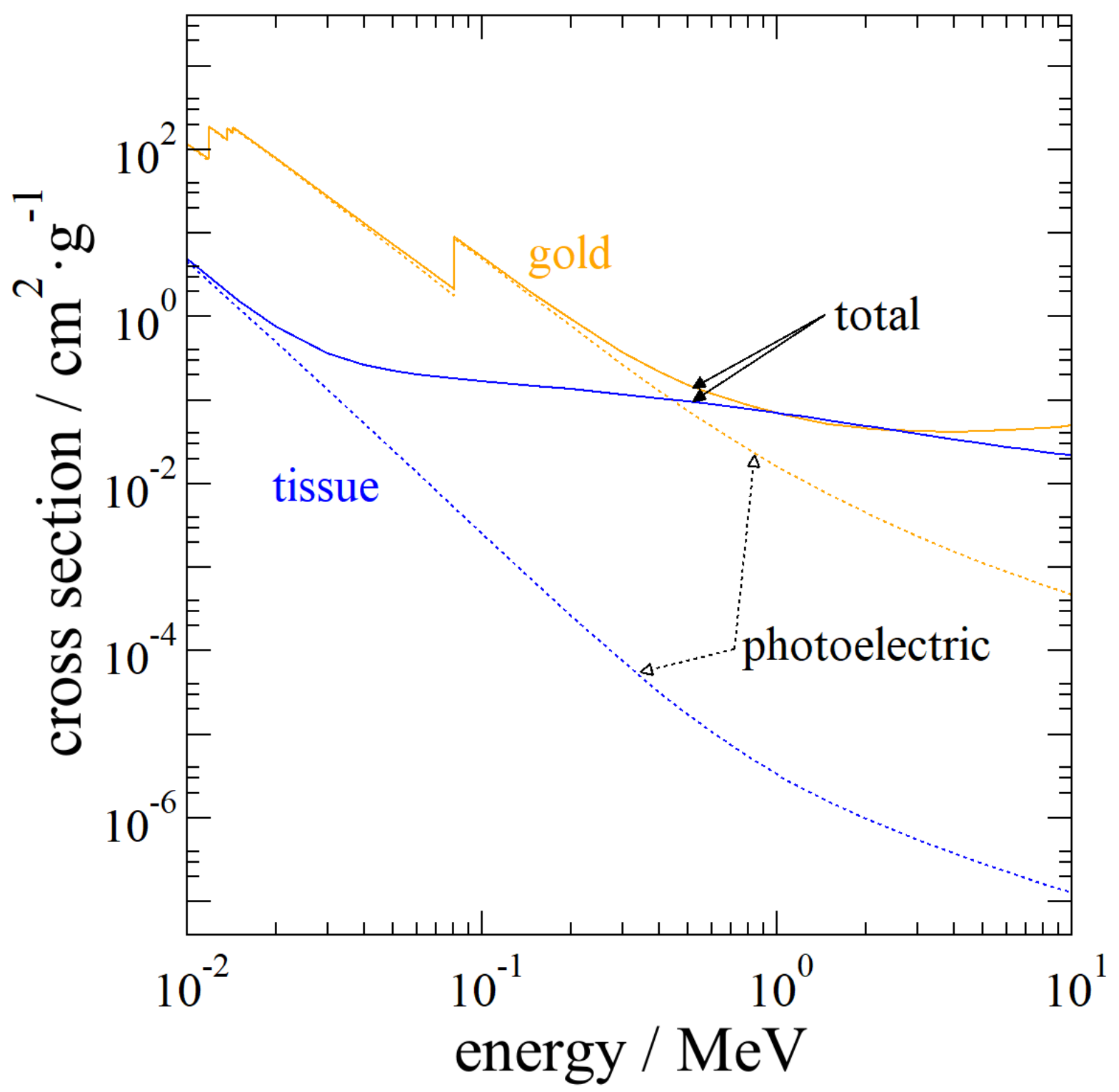

Figure 1.2: Plot of photoelectric and total cross sections as a function of energy for both gold and ICRU four-component tissue. Data is taken from the National Institute of Standards and Technology's XCOM database. ${ }^{18}$ 
Enhancement Factor (DEF), defined as:

$$
\mathrm{DEF}=\frac{\text { dose to tissue in the presence of GNPs }}{\text { dose to tissue in the absence of GNPs }} .
$$

\subsection{Gold nanoparticles for use in radiotherapy}

The work of Hainfield et al in 2004, ${ }^{21}$ using GNPs during irradiation of tumour-bearing mice, was one of the first examples of the use of GNPs as radiosensitizers in vivo, and in it they showed increased survival of mice after undergoing treatment with GNPs. Since then, the use of GNPs as radiosensitizers has been investigated with both in vitro experiments ${ }^{22}$ and computational models. ${ }^{23}$ Recently, there have been a number of new proposed delivery methods, such as adding GNPs to existing brachytherapy treatments ${ }^{24-26}$ and discussion of the viability of using GNPT in the clinic. ${ }^{27}$ To properly translate GNPs to the clinic, the chemical process of GNP synthesis (outlined below) and biological delivery (section 1.4) must be understood and accounted for.

The most common method of GNP synthesis is the Turkevich process,$^{28}$ in which chloroauric acid is treated with citrate to form (roughly) spherical particles over a tunable size (9-120 nm diameter). Chemically reducing the surface tension in the process can alter the morphology of the nanoparticles to be closer in shape to a rod. Gold nanoparticles can also be constructed to have different shapes (e.g., cubes, triangular prisms, wires, and nanocages ${ }^{29}$ ) by using different techniques, such as using silver nanostructures to control GNP morphology by providing a surface template for the Au atoms. ${ }^{30}$ Differing nanoparticle shapes are often sought after for their varying optical properties (surface plasmon resonance) that are useful in imaging techniques such as Raman imaging. ${ }^{29}$

After nanoparticle formation, typically the citrate forms a stable coating around the nanoparticles to maintain their shape, but different solutions can be used to replace the

\subsection{GOLD NANOPARTICLES FOR USE IN RADIOTHERAPY}


stabilising coat. One such coating, PolyEthylene Glycol (PEG) and its different variants are often used for biological applications. ${ }^{31}$

\subsection{Biological aspects of GNPs in tissue}

The most common biological mechanism of GNP delivery is through extravasation (the leaking of GNPs from a blood vessel to the tissue around it), which can occur with $<200 \mathrm{~nm}$ nanoparticles. ${ }^{32}$ Due to the poor vasculature of cancer tumours, they have many leaky blood vessels that allow for GNPs inserted in the blood stream to reach the desired target. There have also been cancer/treatment type dependent delivery methods proposed as well, such as adding GNP eluters to brachytherapy seed strings to release GNPs over the course of a treatment as proposed by Sinha et al. ${ }^{33}$

Properly coated and sized GNPs alone are not toxic to living cells ${ }^{34,35}$ and are metab-

olized through most mammalian organs within a day. ${ }^{36}$ The only concern in the context of GNPT would be unusually high GNP uptake in organs-at-risk near the tumour such as the liver or the spleen, ${ }^{36}$ though these GNPs can be cleared in minutes if they are $<6 \mathrm{~nm}$ in radius. ${ }^{37}$ GNPs accumulate and leave the tumour with time after injection; the highest concentrations/uptakes occur at 30-120 minutes, with a low number remaining after a day, and close to none after a week. ${ }^{36}$

When injected into the tumour, the PEG coating provides a hydrophilic layer around GNPs which avoids macrophages from detecting them and allows them to flow freely through the body. To target cells, the PEG needs to be mixed with biomolecules that can bind with cell surface proteins so that cells absorb (uptake) the GNPs. ${ }^{38}$ Chithrani investigated a wide range of cellular (HeLa cell line) effects during GNP uptake. ${ }^{39}$ GNP uptake can vary significantly with size, with 10 and $100 \mathrm{~nm}$ diameter GNPs typically having the lowest uptake and $50 \mathrm{~nm}$ having the highest (near triple the uptake of 10 or $100 \mathrm{~nm}$ ). Additional 
studies have been performed showing cell-to-cell variation of GNP uptake can vary drastically following a wide distribution, with a significant number of cells with little to no uptake at all. $^{40}$

With typical biomolecule coatings, GNPs get absorbed into membrane-bound compartments (endosomes) in a process called endocytosis until eventually rejected from the cell in exocytosis. ${ }^{39}$ The average time GNPs stay internalized within the cell in this process can vary by cell and GNP shape/size, but can be as short as 20 minutes for $50 \mathrm{~nm}$ diameter, spherical GNPs. Thus, without specialized biomolecules, GNPs will just aggregate into different compartments within the cytoplasm and will not be within the immediate vicinity of the nucleus, leading to a low nucleus DEF. There has been work in the field of GNP synthesis and delivery to extend the duration of the GNP cycle in cells and to better target structures within the cell (such as the nucleus). The GNPs synthesized by Liu et al, ${ }^{41}$ for example, manage to avoid exocytosis for a longer period of time and cluster on the surface of the nucleus, thus are more promising for GNPT.

Most experimental work in GNPT has been performed using either in vitro cell survival studies or small animal survival studies, and has provided useful insight into effects that would need to be accounted for in GNPT. Towards adapting GNPT into clinical treatment, though, a more comprehensive study of DEF in a tumour is required. Experimental techniques for in vivo studies are far more limited, thus most DEF computations for proposed GNPT treatment are performed using MC simulations of radiation transport. 


\subsection{MC simulations of radiation transport}

The Monte Carlo method is a computational approach that uses pseudorandom number sampling to compute numerical solutions. Though there are different MC approaches to radiation transport, this chapter will focus on event-by-event and condensed history MC. ${ }^{42,43}$ MC simulations use cross section data for different media to simulate primary particles (generated from a user-specified radiation source) travelling through a virtual geometry (often called a phantom). One (or more) primary particle(s) sampled from the source is generated and, using cross section sampling and boundary crossing computations, its travel is simulated until it loses all of its energy or leaves the geometry. Any secondary particles (such as knock-on electrons and bremsstrahlung photons) generated with significant kinetic energy are simulated as well. Each simulation of one primary particle sampled from a source is called a history. Many different metrics can be extracted from the simulation of one or more histories, with energy deposition in the geometry being the most common.

Photon transport is performed by sampling (using pseudorandom numbers) photon cross section data appropriate for the medium in which the photon travels to determine photon path length before interaction. The photon is transported along the path until it either reaches the point of interaction or leaves the medium. If entering a new medium in the geometry, a new path length is computed appropriate for the new medium. This approach of modelling each interaction separately is called event-by-event transport or the track structure approach. ${ }^{42}$

Electron transport in modern MC simulations can take two approaches. An event-byevent (track structure) approach, similar to that described above for photons, where the electron interaction cross sections appropriate to the media are sampled and each singlescatter event is simulated discretely. Though an accurate approach and very reliable when computing boundary crossing in a MC geometry, ${ }^{44}$ an electron undergoes a very large number 
of scattering events as it slows and simulating each event can be computationally prohibitive. Thus an alternate model, the Condensed History $(\mathrm{CH})$ approach, is used which simulates several single scatter events in a single step using multiple scattering theories, such as Molière theory. ${ }^{45}$ The energy loss over several interactions is then assumed to be deposited over the path the electron travelled in the step, with most modern MC codes breaking the step into appropriate substeps over which energy deposition is distributed. ${ }^{43}$ There are two classes of $\mathrm{CH}$ algorithms used in MC: class I, where all energy loss of the electron is assumed to be deposited along the track and class II, where secondary particles (i.e., bremsstrahlung and knock-on electrons) are generated along the $\mathrm{CH}$ step and simulated in the history. ${ }^{46}$

Particle transport in $\mathrm{MC}$ codes is performed down to low energies (1 keV or less for most codes), until a particle's energy reaches a cutoff threshold. Radiation transport stops for a particle reaching the cutoff energy or for particles generated at or below the cutoff energy. The remaining energy of the particle is then deposited into the phantom by some calculation, e.g., assumed to be deposited at the point in which it is cutoff as the low-energy particle would be unlikely to leave the scoring region with its short range.

The dose distribution for the virtual geometry in a simulation is computed as the total tallied dose normalized over all the histories of the simulation. The uncertainty is calculated as the standard deviation of the mean dose deposited in a scoring region over all simulated histories; thus the uncertainty decreases linearly as the square of the number of histories increases. Variance reduction techniques ${ }^{47}$ use different statistical tricks (e.g., simulate more particles interacting in scoring regions and fewer in far scatter regions) to reduce the number of histories needed to achieve the same low uncertainties, thus decreasing required computing time (and increasing simulation efficiency).

All Monte Carlo simulations in this thesis are performed with the National Research Council of Canada's EGSnrc Monte Carlo code. ${ }^{48}$ EGSnrc uses the PRESTA-II ${ }^{46}$ electron transport algorithm, a class II CH approach. EGSnrc does not use the $\mathrm{CH}$ approach when an

\subsection{SIMULATIONS OF RADIATION TRANSPORT}


electron (or positron) is within 3 (by default) mean free paths of the nearest boundary, opting for single scatter (event-by-event) transport instead for more accurate boundary crossing. EGSnrc simulates photon and electron transport down to a $1 \mathrm{keV}$ cutoff, i.e., any photons or electrons at or below $1 \mathrm{keV}$ kinetic energy, whether generated below or slowed down to the threshold, are no longer simulated and their (kinetic) energy is deposited in their local region.

\subsection{MC simulations of GNPT}

Early MC simulations recognized that DEFs can vary with depth in the tumour and saw the need to simulate whole tumour volumes. ${ }^{49-52}$ These studies quantified DEF throughout a tumour volume with gold concentrations typically ranging 7-30 $\mathrm{mg}$ of gold per gram of tissue (denoted $\mathrm{mg} / \mathrm{g}$ ); they found that DEFs varied at different positions within the tumour due to attenuation from the additional gold dispersed throughout it. Simulations were performed using a homogeneous gold-tissue mixture model for the entire tumour as the efficient transport calculations in the model allowed for simulations of large volume. Unfortunately, because MC transport is modelled through a single medium whose physical properties computed as the average of gold and tissue, the energy deposited in the gold-tissue mixture cannot be decoupled into the gold and tissue dose components. The DEFs computed in this scenario can be still useful ${ }^{53}$ but these are values that are less directly linked to the dose enhancement effect of GNPs on tissue. ${ }^{52}$

Jones et $a l,{ }^{54}$ using the MC code EGSnrc, performed some of the first simulations of discretely modelled GNPs. They used the dose kernel around a single GNP to extrapolate dose enhancement on a larger scale. The spatial properties of interactions in GNPs were

further investigated by Leung et $a l^{55}$ using the MC code Geant4. McMahon et $a l^{56}$ calculated the energy deposition around a GNP with Geant4 and used it in a computational model to 
calculate relative biological effectiveness of irradiations on MDA-231 cells. Another study quantifying the dose enhancement around a GNP and extrapolating it to finding the number of GNPs required to double prescribed dose for several radionuclides was performed by Lechtman et $a l^{57}$ using the MC code PENELOPE.

Moving from a single GNP model to a biological model, Douglass et $a l^{58}$ used random cell modelling in Geant4 to determine cell dose sensitivity to the cellular configuration of GNPs for a small volume of cells. Though the work did account for variation in cell and nucleus size, the gold was modelled as a large contiguous mass which corresponded to unrealistically large GNP uptake in the cell. McNamara et $a l^{59}$ performed cell simulations using Geant4 with a (single) cell model consisting of a nucleus and many mitochondria situated in a cell whose cytosol contains (a homogeneous mixture of) cytoplasm and gold, with a mass percentage of either $0.001 \%$ or $1 \%$ gold. This study investigated a (relatively) large range of energies, 0-50 keV, as well as several different elemental compositions. Sung et al ${ }^{60}$ performed similar work using a combination of the MC codes TOPAS and Geant4-DNA to measure mean DEF with changes to cell structure (ellipsoidal vs spherical cell shape and nucleus position) by extracellular discretely modelled GNPs for $150 \mathrm{kVp}$ and $6 \mathrm{MV}$ beams for a $2 \%$ concentration of GNPs by mass.

When performing calculations that measure DEF on a cellular level across tumour sizedvolumes, a two-stage approach ${ }^{16}$ is often used, where a macroscopic clinical beam model is combined with a microscopic model. Cai et $a l^{61}$ performed simulations with the MC code MCNP investigating nucleus and cytoplasm DEFs in a cell model with discrete GNPs in different configurations. Their calculations were split into two different simulations, single GNP irradiations to calculate secondary yield due to GNPs and large scatter simulations calculating dose to each cell compartment (assuming no GNPs), combining the two using a mathematical model. Kirkby and Ghasroddashti ${ }^{62}$ calculated dose to the mitochondria of a cell containing GNPs using PENELOPE. Dose distributions resulting from photons striking 
a GNP (and equivalent water nanoparticle) were calculated to estimate dose gain from GNPs on a microscopic scale. The photon fluence spectra incident on the mitochondria was then scored and combined with the nanoparticle distributions to calculate mean DEF. Both the two-stage approaches discussed here investigated a range of energies and concentrations at a fixed depth.

There have also been a few studies that suggest novel GNPT treatments and perform computations predicting DEFs. Asadi et $a l^{63}$ proposed the introduction of GNPs to eye plaque brachytherapy, using the MC code MCNP5 to quantify increased dose to bulk tissue (no cell models) at several points of interest when adding a 7-30 mg/g concentration of discretely modelled GNPs to the tumour under otherwise typical eye plaque treatment parameters. Brivio et $a l^{23}$ proposed the introduction of GNPs into prostate brachytherapy treatments, using the MC code MCNP6 to calculate dose in the treatment when the prostate is injected with gold solutions (concentrations of 10-66 mg/m, distributed heterogeneously throughout the tumour volume). Ngwa et $a l^{64-66}$ have developed a (non-MC) analytic technique and used it to calculate DEF in new proposed treatments. ${ }^{33,67}$

Many of the above studies, which investigate DEF on a tumour scale for a GNPT treatment, often avoid accounting for all the effects of including GNPs in a treatment volume to overcome computational limitations. Effects such as the increased attenuation due to GNPs in the macroscopic volume, photoelectrons generated by GNPs being self-absorbed or absorbed by neighbouring GNPs, or the GNP configurations on the microscopic levels. A primary tenet of this thesis is to introduce a generic model which can be adapted for any GNPT scenarios which accounts for all the above effects. 


\subsection{Thesis Purpose}

A common thread in many of these above Monte Carlo studies modelling GNPs in different scenarios is that they do not fully account for both the macroscopic (tumour scale) and the microscopic (GNP scale) effects anticipated in GNPT. ${ }^{16}$ This thesis focuses on bridging that gap, extracting microscopic dose metrics across macroscopic scales in GNPT. To simulate GNPT on multiple scales, the Heterogeneous MultiScale (HetMS) model is introduced. The HetMS approach is the combination of distinct models of varying level of detail on different length scales within a single simulation; multiscale refers to the multiple models of the same material on different length scales. HetMS simulations of GNPs are performed by combining two different modelling approaches within a single simulation: (1) for most of the phantom's volume, GNPs in tissue are modelled as a homogeneous gold-tissue mixture where transport calculations are very fast but energy deposition to gold and tissue cannot be decoupled; (2) in regions of interest, GNPs are simulated discretely so that desired microscopic metrics can be extracted. Though the HetMS model will only be used in EGSnrc and applied to GNPT, it is defined in a generic (not MC code specific) and can be used for any simulations that seek to extract microscopic dose metrics across much larger scales.

To render simulations of GNPT in EGSnrc feasible with available computational resources, several changes to the code are made. The egs_chamber application, ${ }^{68}$ an application with several variance reduction techniques designed to efficiently score dose to a single cavity, is modified to score dose in multiple separate regions instead. Cubic and hexagonal lattice geometries are added to the egspp geometry library ${ }^{69}$ to enable simulation of a vast number of GNPs and cells efficiently. These changes are described in chapter 2, where the changes, as well as EGSnrc's ability to accurately model radiation transport on micro- and nano-metre length scales, are tested. Cross-validation is performed with codes Geant4-DNA ${ }^{70}$ and PENELOPE. ${ }^{53}$ An electron Fano cavity test is performed on several cell geometries which contain GNPs. 
In chapter 3, the macroscopic effects of adding GNPs at a tumour level are investigated using the HetMS model. The bulk of the tumour volumes are modelled as a gold-tissue mixture and regions of interest are modelled as a lattice of GNPs. In regions of interest, only dose to tissue is scored. This model is used to determine the DEF (to tissue with dispersed GNPs throughout) as a function of depth, exhibiting many dosimetric effects of injecting a large number of GNPs into a treated volume. A similar HetMS approach is also used to simulate a brachytherapy scenario, proposed by Sinha et $a l,{ }^{33}$ where the effects of GNPs on DEF throughout the tumour is investigated. Photon energies ranging $20 \mathrm{keV}$ ( brachytherapy energies) to $1 \mathrm{MeV}$ and gold concentrations of 5,10 and $20 \mathrm{mg} / \mathrm{g}$ are investigated.

A simple cell (nucleus and cytoplasm modelled as concentric spheres) is used to investigate the biological aspects of GNP uptake described in section 1.4. Different methods of modelling GNPs within cells are investigated, towards accurate and efficient cell simulations. Variation in cell size, cell GNP uptake, and GNP distribution throughout cells is investigated for different concentrations and photon source energies (10-370 keV with select $1 \mathrm{MeV}$ simulations) in chapter 4, the energy ranges at which gold and tissue cross sections differ the most (as shown in figure 1.2).

Variation in cell DEF with fluctuations in the gold uptake of a cell and its neighbours is calculated in chapter 5. These variations, as well as the variation with cell/nucleus size, are amalgamated into an expected range of cell DEFs for a given photon source energy (10-370 keV), gold concentration (5-20 mg/g) and GNP configuration within the cell. The cell model is then used to in combination with a large cylinder in a HetMS simulation to compute cell DEF on a tumour scale; results are compared to equivalent DEFs for GNPs dispersed in tissue. Cell DEF (along with the amalgamated expected range of DEFs) as a function of depth is computed. The HetMS sphere simulation of the brachytherapy scenario is revisited using the cell model. 
A summary of the thesis with potential future applications of the heterogeneous multiscale model, in conjunction with the validated and tested EGSnrc are described in chapter 6, within and beyond the scope of GNPT. 


\section{Chapter 2}

\section{EGSnrc simulations at low energies and short length scales}

\section{$2.1 \quad$ Introduction}

This chapter describes the changes made to the EGSnrc code for this thesis and the choice of transport parameters used throughout the thesis. To ensure EGSnrc simulations of cellular dosimetry and the transport of radiation through GNPs remains accurate, cell and GNP simulations are compared with independent simulations performed with different MC codes published in the literature. The electron Fano cavity test, a self-consistency check of radiation transport, is performed on a geometry combining a cell model with $25 \mathrm{~nm}$ radius GNPs.

The primary goal of the changes is to speed up transport calculations in EGSnrc towards creating multiscale simulations of GNPT that complete within reasonable computation times, simulations which include both explicit cell and GNP models throughout a tumoursized volume. The application egs_chamber, ${ }^{47}$ which has many useful variance reduction techniques to increase simulation efficiency, is modified to be able to score dose to multiple regions at once. Without the modifications, egs_chamber would only be able to score a 
total dose value to a list of regions; egs_chamber was originally written to perform fast ion chamber (single dose) calculations. Cubic and hexagonal lattice geometries are implemented using Bravais lattice formalisms. ${ }^{71}$

Cross-validation of the modified EGSnrc and the added geometries is performed. Cellular dosimetry in a Medical Internal Radiation Dosimetry scenario is performed and compared with Geant4-DNA results. Dose to the tissue in the presence of GNPs divided by dose to an equivalent gold-tissue mixture is computed and compared with PENELOPE results.

Beyond the comparison with other Monte Carlo codes, EGSnrc is also verified with the electron Fano cavity test. ${ }^{2}$ The Fano test, based on Fano's theorem, ${ }^{73}$ is considered a major requirement for $\mathrm{MC}$ codes of radiation transport, as it sets up a scenario in which dose calculations can be benchmarked against an analytical dose expression. Thus, the Fano cavity test provides an additional check on EGSnrc's ability to model transport through complex geometries independent of other work. A geometry consisting of a cell containing GNPs is tested to ensure dose prediction is still within the $0.1 \%$ threshold for EGSnrc established previously. ${ }^{74,75}$

\subsection{Methods}

MC simulations are carried out using the EGSnrc ${ }^{48}$ distribution with the egspp class library ${ }^{69}$ (in the current and all following chapters). Modifications to EGSnrc were first performed on the 2015 release of EGSnrc (which was used for all simulations in chapter 3) and then updated to commit 822ec3a of the EGSnrc github develop branch (used for all other simulations in the thesis). The application egs_chamber ${ }^{47}$ is used due to its variance reduction techniques (used in later chapters), but is modified to enable energy-deposition scoring in multiple regions. 
Transport parameters used are generally the EGSnrc defaults with the following exceptions: pair angular sampling is turned off, Rayleigh scattering and electron impact ionization are turned on, NRC cross section data are used for bremsstrahlung events and XCOM cross section data ${ }^{18}$ are used for photon interactions. Explicit $M$ - and $N$-shell transitions are modelled to account for the dosimetric effects of these atomic relaxations ${ }^{76}$ (EGSnrc default is to treat $M$ - and $N$-shell transitions in an average way); note that $K$ - and $L$-shells are considered explicitly by default. Photons and electrons are simulated down to $1 \mathrm{keV}$ kinetic energy. The high resolution random number generator option is enabled. All media used in the MC simulations in this thesis are either gold, water, ICRU four-component tissue $(10.1 \%$ hydrogen, $11.1 \%$ carbon, $2.6 \%$ nitrogen, and $76.2 \%$ oxygen by mass ${ }^{20}$ ), or a homogeneous blend of gold with tissue or water.

\subsubsection{Lattice geometry implementation in EGSnrc}

Two lattice geometry classes are created to efficiently model GNPs and cells within a medium discretely, a cubic and a hexagonal close-packed lattice. Cubic lattices in MC have been em-

ployed in other studies, ${ }^{77-81}$ but are not included as part of the egspp geometry class library; ${ }^{69}$ this subsection describes the implementations of egspp lattice geometries in EGSnrc.

The cubic (and hexagonal) lattice geometry take a predefined geometry, referred to as the subgeometry, and place it at points defined by one (or four) Bravais lattices ${ }^{71}$ within a chosen region of another geometry, referred to as the holder. A vector representing a point along a Bravais lattice is defined as

$$
\vec{r}(i, j, k)=i a_{x} \hat{x}+j a_{y} \hat{y}+k a_{z} \hat{z}
$$

where $a_{x}, a_{y}, a_{z} \in \mathbb{R}$ and $i, j, k \in \mathbb{Z}$

for any arbitrary indices $i, j$, and $k$. The same instance of the subgeometry (i.e., you can 
only score in all of them or none of them) is placed at all possible positions $\vec{r}$ within a region of the holder. The lattice geometries provide substantial reductions in memory use (only store one subgeometry in memory) and radiation transport time (only transport through subgeometries whose $\vec{r}$ is close to photon or electron path) compared to a simulation in which each subgeometry would be modelled explicitly.

A cubic lattice is defined by setting $a_{x}, a_{y}$, and $a_{z}$ in equation (2.1) to a single centreto-centre distance $a$. The subgeometry then repeats in each of the $x, y$ and $z$ directions with the same spacing $a$ around a subgeometry defined at the origin (though this can be translated with other egspp geometry classes), as shown in figure 2.1. In EGSnrc, there are three requisite functions which perform the majority of geometry computations for particle transport: howfar (), hownear(), and isWhere(). is Where() gets passed a position $[x, y, z]$ and returns the geometry region in which that point lies. In the case of the lattice, when the point is in a holder region that contains subgeometries, a virtual subgeometry is placed at the nearest position to that point (i.e., $a[\operatorname{round}(x / a), \operatorname{round}(y / a), \operatorname{round}(z / a)])$ and the is Where () of the virtual subgeometry is queried. If the point is within the subgeometry, the subgeometry is Where() call is returned otherwise the holder region is Where() is returned.

hownear() returns the distance to the closest boundary from a position $(x, y, z)$. As in the is Where() call, a virtual subgeometry is placed at the closest lattice position to that point, but also the seven neighbouring positions (one index up or down from the nearest subgeometry) which provide the points to define a cube which encloses the initial position. The shortest hownear() call of the eight subgeometries and the holder is returned.

howfar () is a function which (in brief) takes a (particle's) position $[x, y, z]$, direction of motion $[p, q, r]$, and distance it would travel in the medium, and then calculates if the distance fits in the invoked region or how far the particle would travel in the region before leaving. If the call is in the holder region with subgeometries, the algorithm draws the track from the start position until it travels the full distance or leaves the holder region. At every point that 


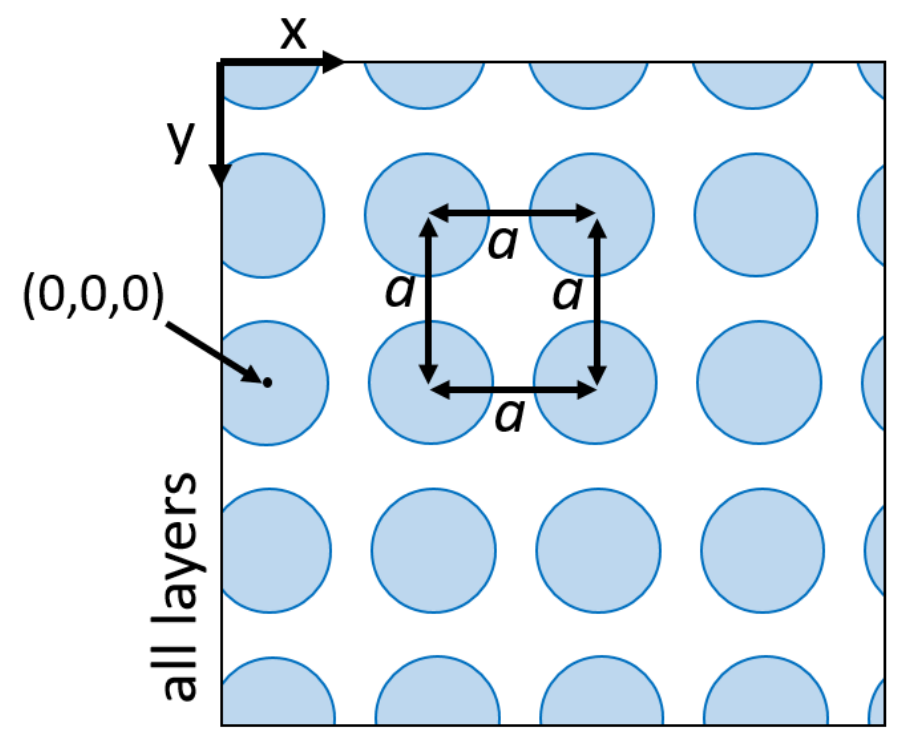

Figure 2.1: 2D cross-sectional diagram showing an arbitrary slice of a cubic lattice layer of spheres with center-to-center distance $a$.

the track intersects with planes defined at $x=n \cdot \frac{a}{2}, y=n \cdot \frac{a}{2}$, and $z=n \cdot \frac{a}{2}$ as well as the starting and ending track positions, a point on the track is defined (see figure 2.2). For each defined point along the track (ordered from start to end), a virtual subgeometry is placed at the nearest lattice position to that point and howfar () is invoked for that subgeometry. The algorithm stops iterating if there is an intersection with a subgeometry or returns the howfar() of the holder if no intersections are found. This boundary crossing algorithm is exact if the subgeometries are centred at their lattice positions and if any radial asymmetries in the subgeometry boundary are not larger than a quarter of the centre-to-centre distance a. Any implementation in this thesis only uses subgeometries with spherically symmetric boundaries. Appendix A contains the full cpp implementations of the howfar (), hownear(), and is Where() cubic lattice functions.

The cubic lattice is a (relatively) simple and efficient implementation of radiation transport but does not allow for close enough packing of cells to achieve common cell densities. ${ }^{82}$ Thus a hexagonal close-packed lattice is developed using four overlapping Bravais lattices 


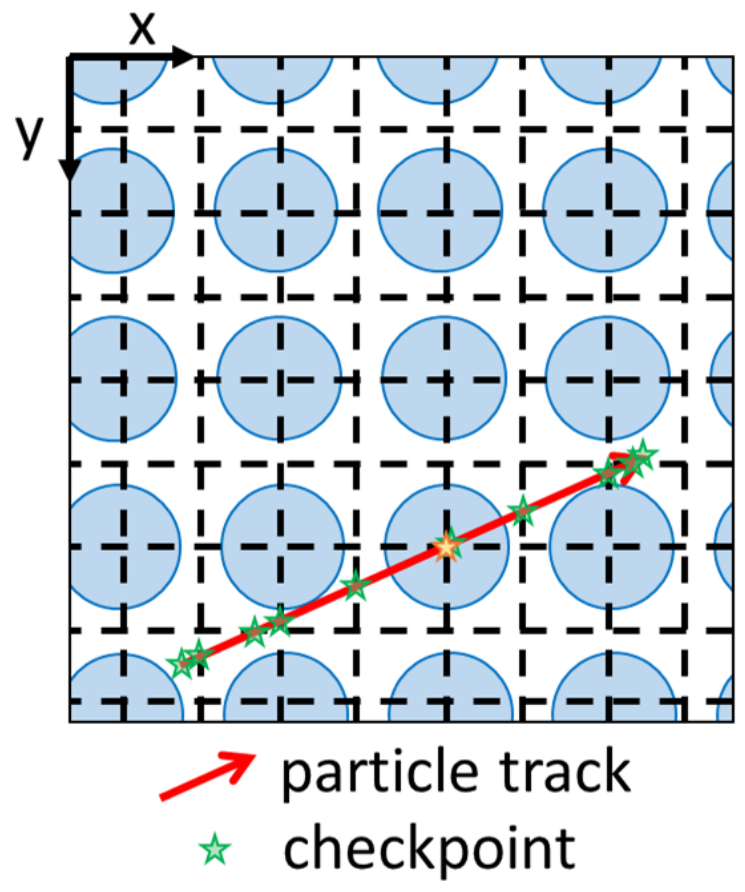

Figure 2.2: Diagram depicting a 2D example of cubic lattice howfar() algorithm. The red line (starts bottom left, finishes top right) is the trajectory for which howfar () is invoked and the green stars are the positions (checkpoints) at which the howfar() algorithm of the nearest subgeometry is called in the same order as the trajectory direction. The gold star is the first checkpoint that returns an intersection result from the nearest subgeometry. 
(see figure 2.3) to achieve smaller centre-to-centre distances $(a)$ between subgeometries. The transport algorithms for the hexagonal lattice is an amalgamation of transport through a Bravais lattice $\left(\overrightarrow{r_{1}}\right)$ and its three translations $\left(\overrightarrow{r_{2}}, \overrightarrow{r_{3}}\right.$, and $\left.\overrightarrow{r_{4}}\right)$, defined as

$$
\begin{aligned}
& \overrightarrow{r_{1}}(i, j, k)=\quad i a \hat{x}+\quad j \sqrt{3} a \hat{y}+\quad k \sqrt{3} a \hat{z} \\
& \overrightarrow{r_{2}}(i, j, k)=\left(i+\frac{1}{2}\right) a \hat{x}+\left(j+\frac{1}{2}\right) \quad \sqrt{3} a \hat{y}+\quad k \sqrt{3} a \hat{z} \\
& \overrightarrow{r_{3}}(i, j, k)=\quad i a \hat{x}+\left(j+\frac{1}{4}\right) \quad \sqrt{3} a \hat{y}+\left(k+\frac{1}{2}\right) \quad \sqrt{3} a \hat{z} \\
& \overrightarrow{r_{4}}(i, j, k)=\left(i+\frac{1}{2}\right) a \hat{x}+\left(j-\frac{1}{4}\right) \quad \sqrt{3} a \hat{y}+\left(k+\frac{1}{2}\right) \quad \sqrt{3} a \hat{z} \\
& \text { where } a \in \mathbb{R} \text { and } i, j, k \in \mathbb{Z}
\end{aligned}
$$

The code implementation is similar in structure to the cubic lattice transport described above with a few additional optimizations in place for this scenario. Appendix B contains the full cpp implementations of the howfar(), hownear (), and is Where() hexagonal lattice functions. The lattices are defined by volumetric number density, the class constructor calculates the appropriate centre-to-centre distance $a$.

In simulations for which a lattice is irradiated by a parallel beam, the lattice is rotated $\left(15^{\circ}\right.$ about one perpendicular axis and $30^{\circ}$ about the other) relative to the beam axis to avoid shadowing (i.e., having all primary photon trajectories either intersecting with all subgeometries in a row or none at all) which would create a bias that would not exist in a random distribution. Results in this study are not sensitive to the angles chosen as long as the lattice axes of symmetry are not close to parallel (within $3^{\circ}$ ) with the beam. 


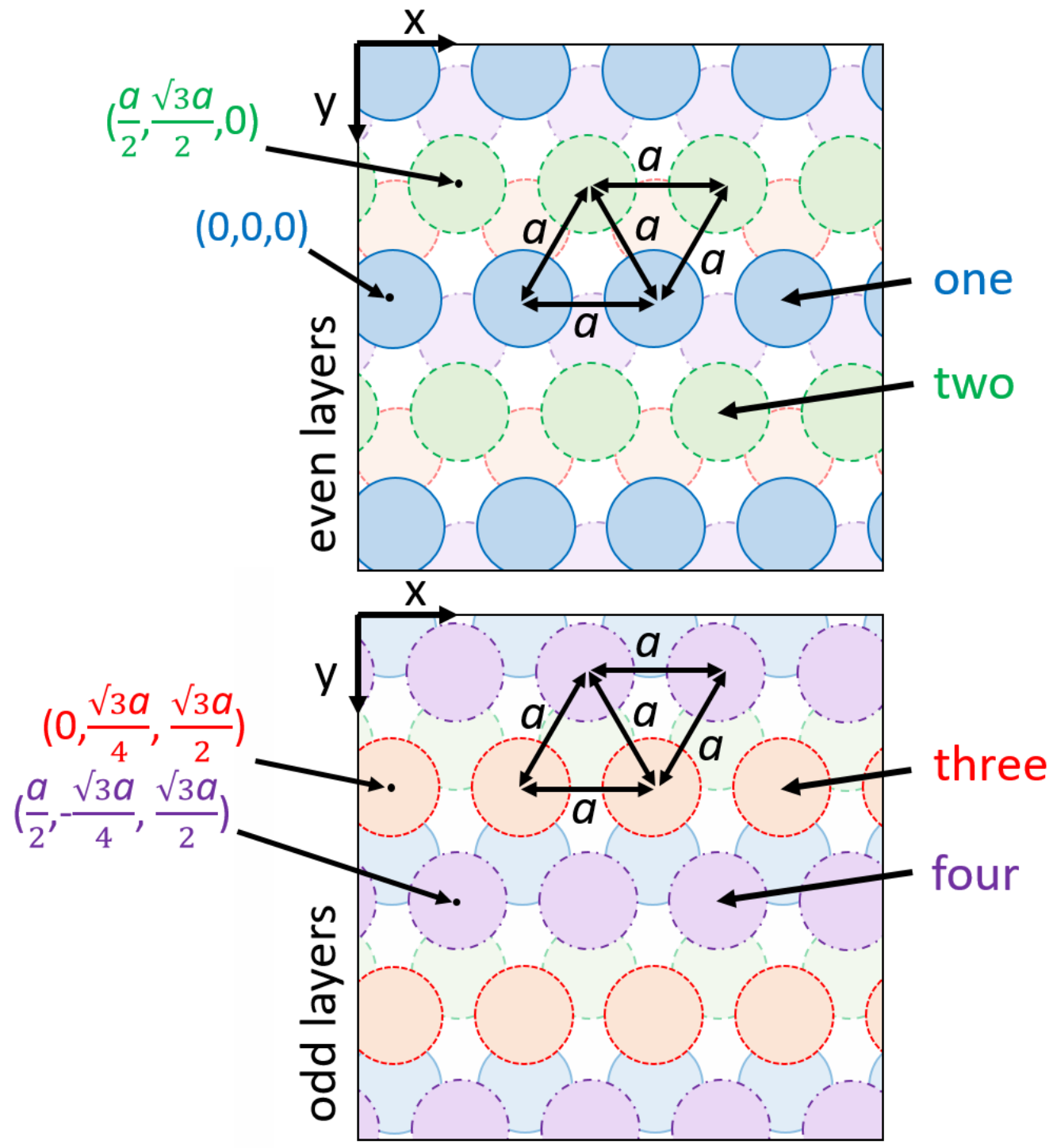

Figure 2.3: Diagrams depicting a 2D example of two hexagonal close-packed lattice layers of spheres with centre-to-centre distance $a$. The four different Bravais lattices defined by equation (2.2) are shaded and outlined separately for clarity. 


\subsubsection{Cross-validation of EGSnrc}

\section{Scoring dose in cellular geometries}

EGSnrc's ability to score dose to cellular sub-compartments (i.e., nucleus and cytoplasm) is compared with the Geant4-DNA results of Šefl et al. ${ }^{70}$ Medical Internal Radiation Dose (MIRD) S-value calculations are performed on a cell modelled as two concentric spheres, an inner nucleus and outer cytoplasm, with dimensions $\left(r_{\text {cell }}, r_{\text {nuc }}\right)=(5,4) \mu \mathrm{m}$. Isotropic, monoenergetic electron sources are distributed in four different configurations: on the surface of the cell, throughout the entire cell, throughout only the cytoplasm or throughout only the nucleus. Doses to the nucleus and cytoplasm are scored for many electron energies ranging from 1 to $100 \mathrm{keV}$ and are used to calculate S-values for four different scenarios where the S-value is the dose absorbed by a target (i.e., the nucleus, cytoplasm or both) per activity per second of a specific source configuration. S-values are therefore equivalent to dose/history as the number of histories and electrons are the same. All cell compartments are modelled as liquid water. Four source/target scenarios, outlined in figure 2.4, are simulated

and compared with calculations using Geant4-DNA. Each scenario is simulated to $4 \times 10^{9}$ histories to achieve sub-0.01\% uncertainty for all but some low-energy cases.

\section{Simulation of nanometre geometries}

Validation of MC transport through GNPs is performed by comparison with the (independent) results of Koger and Kirkby. ${ }^{53}$ In their work, monoenergetic photons or electrons incident on microcavities filled with either randomly-distributed spherical GNPs $(2,10,20$, 50 and $100 \mathrm{~nm}$ diameter) embedded in ICRU four component tissue ${ }^{20}$ or a homogeneous mixture of gold and tissue were modelled using PENELOPE. Photons and electrons were simulated down to $100 \mathrm{eV}$, and recursive boundary conditions were established. Ratios of the dose-to-tissue relative to dose to the homogeneous gold-tissue mixture were published (to 

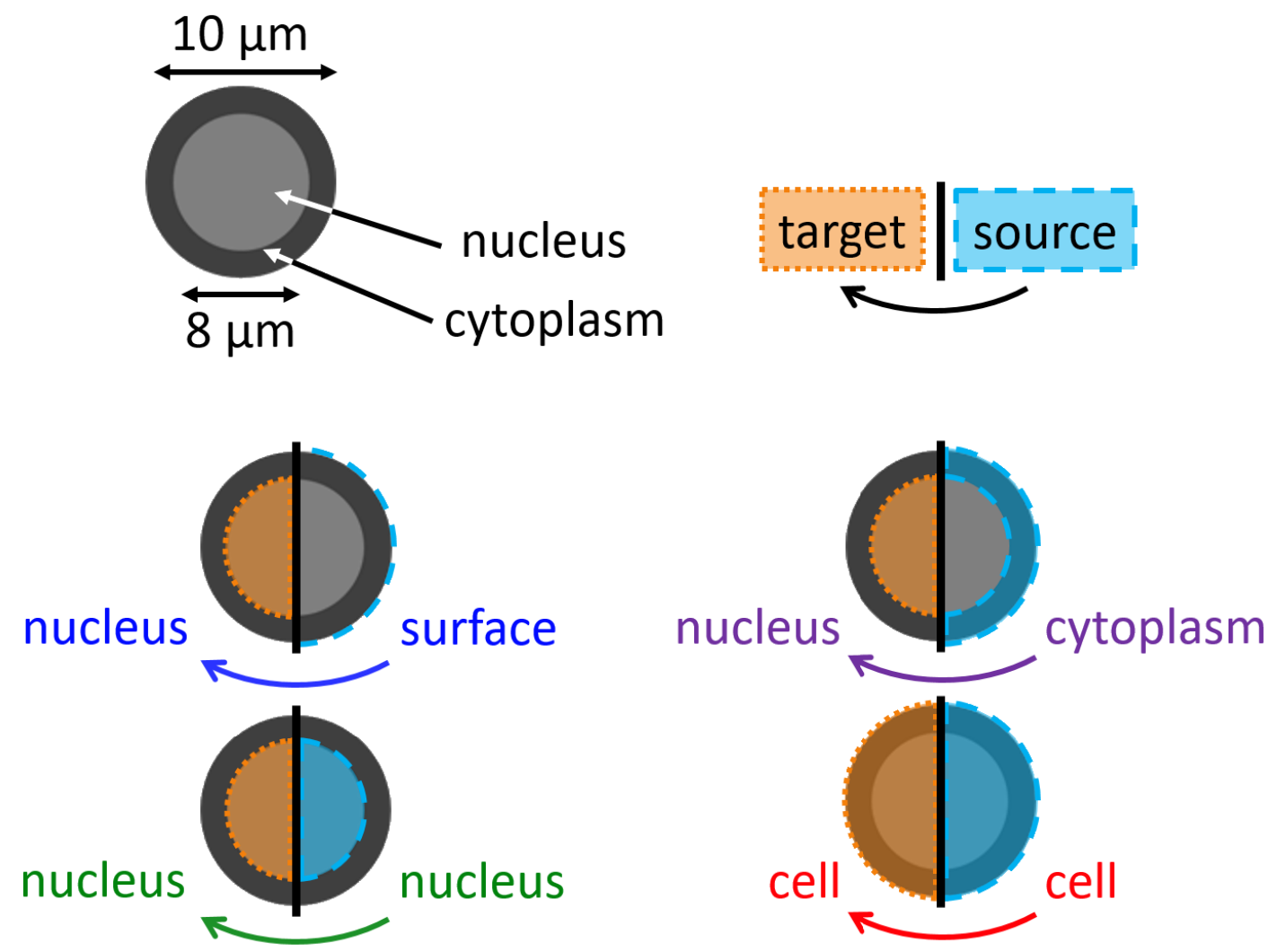

Figure 2.4: Cross section diagram of the cell used for the S-value calculations (top) and the four scenarios (labelled as target $\leftarrow$ source) compared to Geant4-DNA. 
allow conversion of dose scored in a mixture to dose-to-tissue) for a range of source energies and GNP concentrations.

In the current work, dose ratios (dose-to-tissue relative to dose-to-mixture of tissue and gold) are calculated with simulations involving cylindrical geometries. A monoenergetic (20, 30 or $50 \mathrm{keV}$ ) parallel photon beam (circular cross section, $150 \mu \mathrm{m}$ radius) is incident on a cylinder (150 $\mu \mathrm{m}$ radius, $200 \mu \mathrm{m}$ long) containing either a lattice (cubic or hexagonal) of GNPs (20, 50 or $100 \mathrm{~nm}$ diameter) embedded in pure ICRU tissue or a homogeneous goldtissue mixture (concentrations of 5, 10 and $20 \mathrm{mg} / \mathrm{g}$ ). Dose is scored in a smaller cylinder (100 $\mathrm{mm}$ radius, $100 \mu \mathrm{m}$ long) located at the centre of the larger cylinder (see figure 2.5), with dimensions matching the microscopic scoring cylinders used in chapters 3 and 5 . The dose ratio (or conversion factor) is computed by taking the ratio of the dose to only tissue in the GNP lattice scenario over dose to the mixture in the homogeneous scenario. Simulations are run for $10^{10}$ histories for most cases, $2 \times 10^{10}$ for $20 \mathrm{keV}$ simulations.
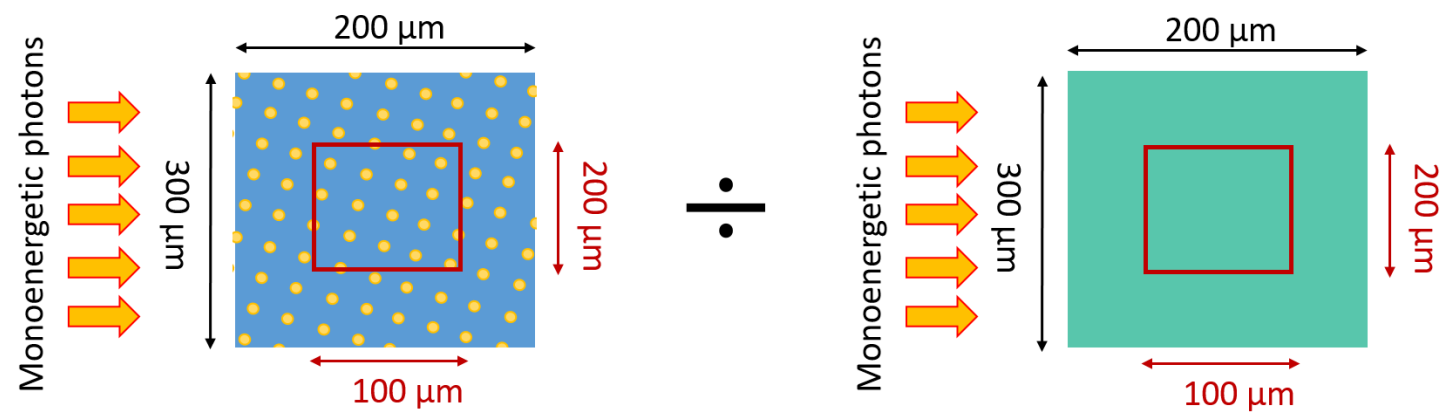

Figure 2.5: Cross section diagram of the cylindrical phantom comprised of a lattice of GNPs embedded in tissue (left) and a gold tissue mixture (right); the scoring region, outlined in red, is at the centre of the larger phantom. 


\subsubsection{Electron Fano cavity test}

An electron Fano cavity test, based on work presented by Sempau and Andreo ${ }^{83}$ and Bouchard et $a l^{72}$ for testing transport in magnetic fields, is performed (with zero magnetic field) to test electron transport in EGSnrc. A single cell model with a hexagonal lattice of GNPs in a thin shell (figure 2.6), whose context and motivation will be explained in chapter 4 , is used for the test. The cell is placed in a small cube of tissue (side length $25 \mu \mathrm{m}$ ), with uniform material elemental composition (ICRU tissue), but non-uniform mass density (i.e., GNPs are modelled as spheres of $19.32 \mathrm{~g} / \mathrm{cm}^{3}$ density tissue with a density effect correction for nominal tissue density) to maintain CPE everywhere. The source is an isotropic electron source throughout the cell and cube generating the same number of electrons per unit mass everywhere (i.e., 19.32 times as many electrons per unit volume in the GNPs as elsewhere). Recursive boundary conditions are established; if a particle crosses the $\mathrm{x}, \mathrm{y}$ or $\mathrm{z}$ boundaries of the cube, it is translated to the opposite boundary while maintaining its velocity. The recursive boundary condition is fulfilled by using the test cell as the subgeometry in a cubic lattice with a centre-to-centre distance of $25 \mu \mathrm{m}$.

In Charged Particle Equilibrium (CPE) conditions, with the source used in this simulation, the dose per history calculation is very simple, i.e.,

$$
\begin{aligned}
\text { dose } & =\frac{\text { electron energy } \cdot \text { number of electrons }}{\text { Fano cube mass }} \\
\frac{\text { dose }}{\text { history }} & =\frac{\text { electron energy }}{\text { Fano cube mass }}
\end{aligned}
$$

because the number of histories is the number of generated electrons. The above MC scenario should thus compute the exact value described in equation (2.3). Three cell sizes are tested: $\left(r_{\text {cell }}, r_{\text {nuc }}\right)=(5,3) \mu \mathrm{m},(7.35,5) \mu \mathrm{m}$, and $(10,8) \mu \mathrm{m}$. Source energies of 20,30 and $50 \mathrm{keV}$ are used and a gold concentration of $20 \mathrm{mg} / \mathrm{g}$ (25 nm GNP radius) is considered. Tests are run for $10^{9}$ histories in most cases, except for $2 \times 10^{9}$ histories for the small cell at $30 \mathrm{keV}$ 
and $4 \times 10^{9}$ histories for the small cell at $20 \mathrm{keV}$, to achieve sub $0.01 \%$ uncertainties.

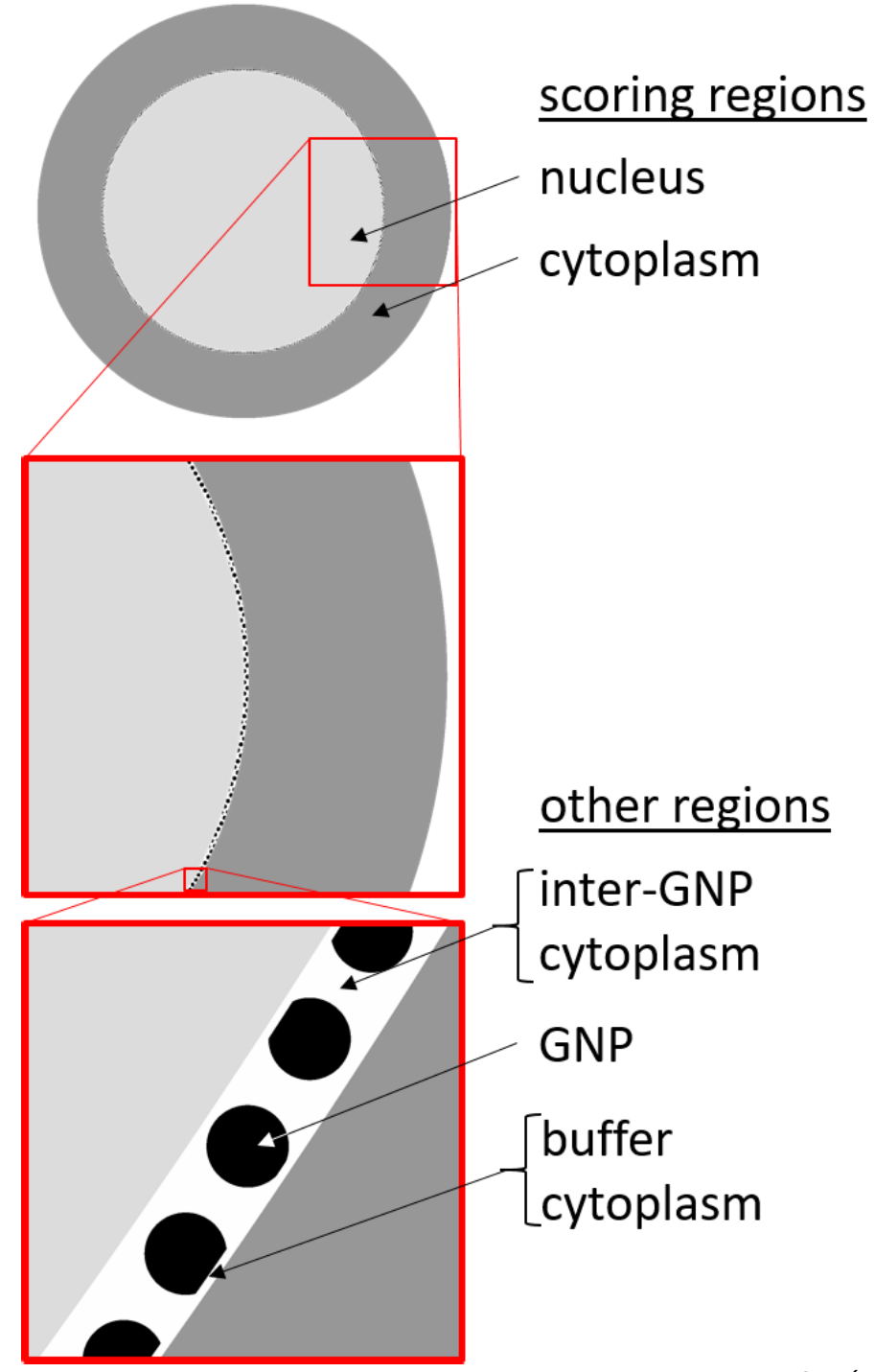

Figure 2.6: EGSnrc-generated images, using application egs_view, of a $\left(r_{\text {cell }}, r_{\text {nuc }}\right)=(7.35,5) \mu \mathrm{m}$ for a $20 \mathrm{mg} / \mathrm{g}$ gold concentration used in the electron Fano cavity tests. GNPs have a radius of $25 \mathrm{~nm}$. 


\subsection{Results}

\subsubsection{Cross-validation}

\section{Scoring dose in cellular geometries}

S-values calculations for four different scenarios (figure 2.4) are shown in figure 2.7 and compared with S-values computed by Šefl et $a l^{70}$ using Geant4-DNA. The percent different between the Geant4-DNA results with those using egs_chamber is on average 1.8\% with a median difference of $0.60 \%$ (excluding sub $0.1 \mathrm{mGy} / \mathrm{Bq} / \mathrm{s}$ S-values as the doses are very low with high uncertainty). The majority of S-values agree to sub $1 \%$ levels, with a few outliers for sub-25 keV energy S-values in the nucleus $\leftarrow$ cytoplasm scenario.

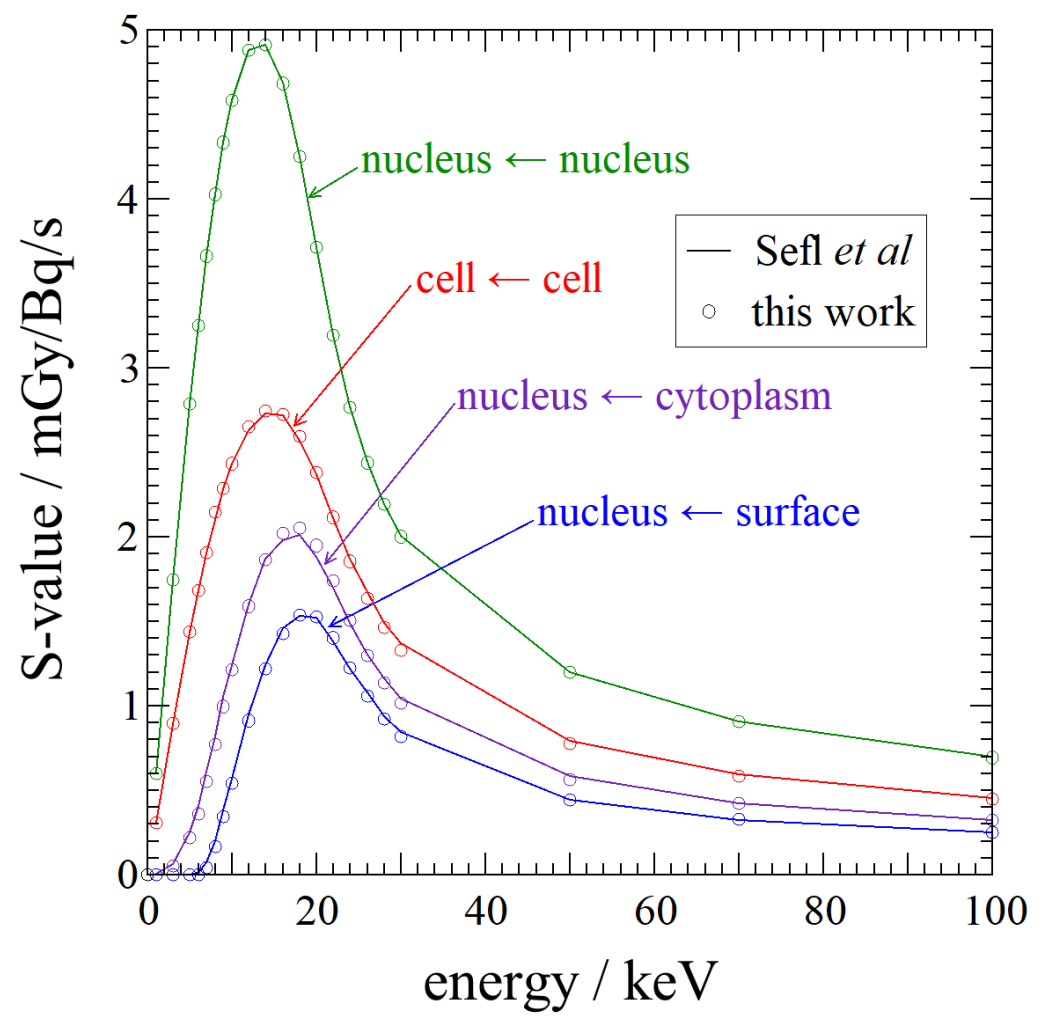

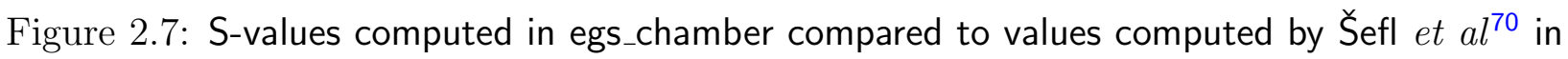
Geant4-DNA for 4 different scenarios (target $\leftarrow$ source). 


\section{Simulation of nanometre geometries}

Table 2.1 presents dose ratios (dose-to-tissue relative to dose to a homogeneous tissue-gold mixture) for various gold concentrations and source energies using a cubic lattice model for GNPs. Simulations with a hexagonal lattice instead of a cubic lattice agreed within statistical uncertainties. While many values computed in the current work agree with the published values of Koger and Kirkby ${ }^{53}$ within the $1 \sigma$ statistical uncertainties indicated, most values agree within close to $2 \sigma$ uncertainties. There is a notable outlier in the $20 \mathrm{mg} / \mathrm{g}$ concentration of $50 \mathrm{~nm}$ GNPs at $50 \mathrm{keV}$ conversion factors. It is of note that the Koger and Kirkby result in this case are much closer to unity than in the 10 or $5 \mathrm{mg} / \mathrm{g}$ cases, which opposes the expected trend (more gold leads to larger discrepancy in models, which leads to conversion factors farther from unity) and is likely a statistical outlier.

Omitting the aforementioned data point, the largest percent difference observed is $2.5 \%$ for the $50 \mathrm{keV}$ beam with $20 \mathrm{~nm}$ diameter GNPs having a concentration of $20 \mathrm{mg} / \mathrm{g}$. The absolute difference between our results and those of Koger and Kirkby averaged over all source energies, GNP diameters, and gold concentrations (except $20 \mathrm{mg} / \mathrm{g}, 50 \mathrm{~nm}$ and $50 \mathrm{keV}$ case) considered is $0.98 \%$, the average absolute difference divided by uncertainty is 0.99 . Simulations repeated with larger cylindrical phantoms (radii of 200 or $300 \mu \mathrm{m}$ rather than $150 \mu \mathrm{m}$, lengths of 300 or $400 \mu \mathrm{m}$ rather than $200 \mu \mathrm{m}$ ) but with the same central scoring volume (radius $100 \mu \mathrm{m}$, length $100 \mu \mathrm{m}$ ) yield dose ratios in agreement within statistical uncertainties. 


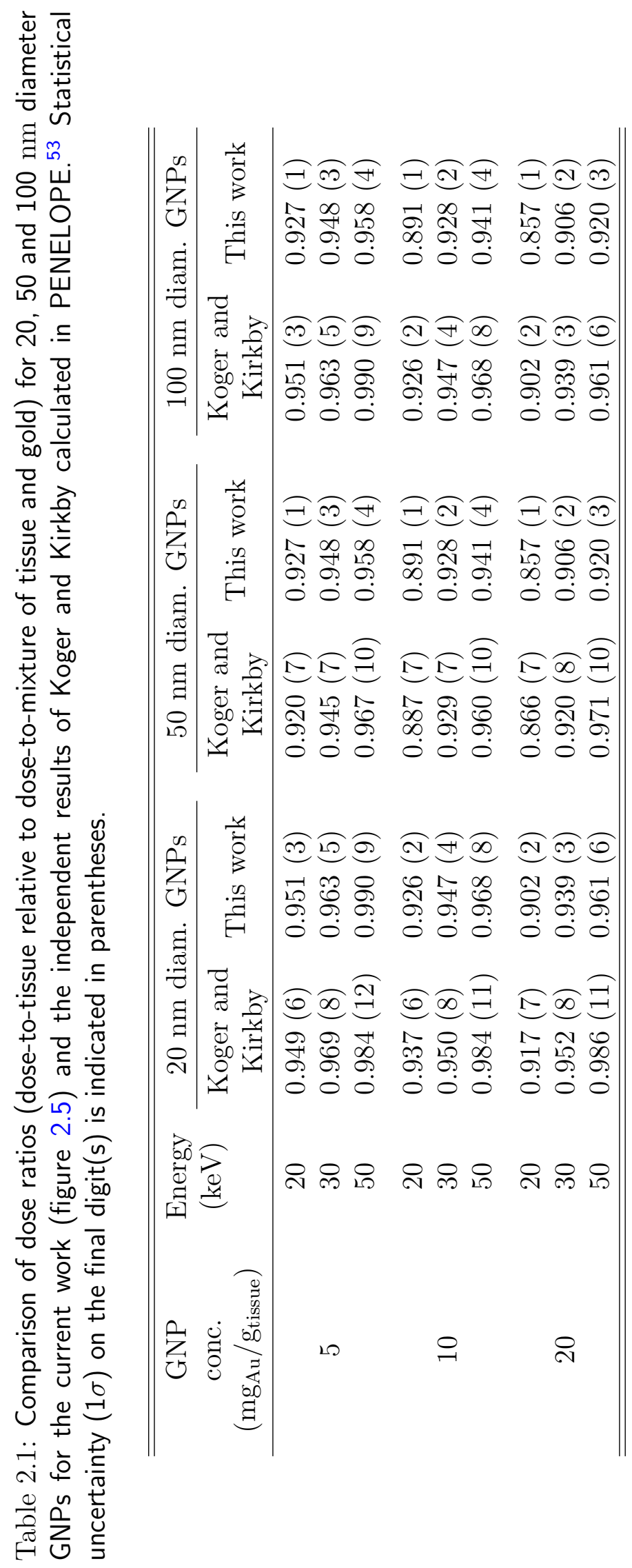




\subsubsection{Electron Fano cavity test}

Results of the Fano test are compiled in table 2.2. For all cell sizes and energies investigated, with dose/history uncertainties computed to $0.01 \%$ or less, are within $0.1 \%$ of the expected value calculated with equation (2.3). This indicates that EGSnrc passes the Fano test to the previously established $0.1 \%$ threshold $^{74,75}$ for a cell model using the new lattice geometries. The $0.1 \%$ threshold is often used for this test as $0.1 \%$ is often taken as the lower limit of many of the inherit uncertainties used in EGSnrc simulations, such as uncertainties in electron stopping power. ${ }^{84}$ The calculated value minus the theoretical value is systematically positive.

Table 2.2: Electron Fano cavity test using an isotropic electron source egs_chamber dose calculations ( $0.01 \%$ statistical uncertainty) and comparison to expected dose, $\% \Delta=\frac{\text { result-expected }}{\text { expected }} \cdot 100 \%$

\begin{tabular}{|c|c|c|c|c|}
\hline \multirow{2}{*}{$\begin{array}{c}\left(r_{\text {cell }}, r_{\text {nuc }}\right) \\
\mu \mathrm{m}\end{array}$} & \multirow{2}{*}{ region } & $20 \mathrm{keV}$ & $30 \mathrm{keV}$ & $50 \mathrm{keV}$ \\
\hline & & $\mu \mathrm{Gy} /$ hist $(\% \Delta)$ & $\mu \mathrm{Gy} /$ hist $(\% \Delta)$ & $\mu \mathrm{Gy} /$ hist $(\% \Delta)$ \\
\hline \multirow{3}{*}{$(5,3)$} & nucleus & $204.79(0.01 \%)$ & $307.44(0.09 \%)$ & $512.21(0.06 \%)$ \\
\hline & cytoplasm & $204.83(0.03 \%)$ & $307.39(0.08 \%)$ & $512.20(0.05 \%)$ \\
\hline & expected & 204.77 & 307.16 & 511.93 \\
\hline \multirow[t]{2}{*}{$(7.35,5)$} & $\begin{array}{r}\text { nucleus } \\
\text { cytoplasm }\end{array}$ & $\begin{array}{l}204.28(0.01 \%) \\
204.35(0.05 \%)\end{array}$ & $\begin{array}{l}306.51(0.04 \%) \\
306.56(0.06 \%)\end{array}$ & $\begin{array}{l}510.74(0.02 \%) \\
510.85(0.04 \%)\end{array}$ \\
\hline & expected & 204.25 & 306.38 & 510.63 \\
\hline \multirow[t]{2}{*}{$(10,8)$} & $\begin{array}{r}\text { nucleus } \\
\text { cytoplasm }\end{array}$ & $\begin{array}{l}203.26(0.03 \%) \\
203.36(0.08 \%) \\
\end{array}$ & $\begin{array}{l}304.94(0.05 \%) \\
305.00(0.07 \%) \\
\end{array}$ & $\begin{array}{l}508.19(0.04 \%) \\
508.31(0.06 \%)\end{array}$ \\
\hline & expected & 203.20 & 304.79 & 507.99 \\
\hline
\end{tabular}




\subsection{Discussion}

S-value calculations performed with EGSnrc (electron cutoff energy of $1 \mathrm{keV}$ ) have good agreement with the calculations of Sefl et $a l^{70}$ (Geant4-DNA, $7.4 \mathrm{eV}$ cutoff) for source energies ranging 1-100 keV. However, there is a notable discrepancy in the nucleus $\leftarrow$ cytoplasm scenario, for which differences are above $2 \%$ for energies ranging $14-24 \mathrm{keV}$. This discrepancy is likely not caused by the difference in electron energy cutoffs, as lower energy S-values agree and the nucleus $\leftarrow$ surface scenario does not exhibit the same discrepancies. The suspected cause of the discrepancy is the non-exact boundary crossing that can occur in Geant4 when electron transport parameters are not carefully defined ${ }^{85}$ when generating (and thus transporting) electrons very close to the nucleus boundary. Omitting nucleus $\leftarrow$ cytoplasm S-values from the statistics, the median deviation between Geant4-DNA and EGSnrc is only $0.4 \%$.

The demonstrated agreement between our results and those of Koger and Kirkby ${ }^{53}$ (table 2.1) from their PENELOPE (electron cutoff energy of $100 \mathrm{eV}$ ) simulations provides validation of EGsnrc's ability to simulate radiation transport through GNPs and to score dose to microcavities containing GNP, demonstrating the appropriateness of EGSnrc with its $\mathrm{CH}$ approach (class II scheme ${ }^{46}$ ), as well as its $1 \mathrm{keV}$ transport cutoff and lattice geometry, towards cellular dosimetry in GNPT. While use of such CH codes and $1 \mathrm{keV}$ transport cutoffs enhance simulation efficiency, thus making simulations possible with available computing power, some GNPT applications require consideration of electron transport to lower energies and event-by-event simulation of electron transport (with various associated challenges $\left.{ }^{16,86,87}\right)$. These applications include quantification of energy deposition with $\sim \mathrm{nm}$ resolution in the immediate vicinity of GNPs towards understanding possible considerable DEF variability on $\mathrm{nm}$ length scales. ${ }^{88,89}$

MC simulations in low-energy regimes encounter other limitations beyond cutoff en- 
ergies. The error on stopping powers used in EGSnrc below $10 \mathrm{keV}$ varies from 10\% (for low-Z material) to much higher for some high-Z material while still above the $1 \mathrm{keV}$ cutoff. $^{90}$ Photon cross section data are potentially a source of higher uncertainties; the Livermore Evaluated Photon Data Library used for PENELOPE and Geant4-DNA physics lists up to $200 \%$ uncertainties for photon cross sections down to $100 \mathrm{eV}^{91}$ in solid water. Fortunately, particles with energies on the order of a few $\mathrm{keV}$ deposit a relatively small fraction of the overall energy in simulations in which particles are initiated at $\geq 20 \mathrm{keV}$ energies, i.e., the majority of energy deposition events occur using data with low uncertainties. Taking the ratio of doses in two different simulations also has the potential to reduce uncertainties from cross section data, as discrepancies can cancel out.

Beyond the cross-validation, the electron Fano cavity test (section 2.3.2) on a cell containing GNPs shows transport consistency results with EGSnrc when tested against analytical dose calculations. The Fano test demonstrates two things when simulating GNPs in EGSnrc, (1) the $1 \mathrm{keV}$ cutoff does not impact measured cellular dose values which are within $0.1 \%$ of their expected value even if the cell contains GNPs and (2) EGSnrc's electron transport algorithm maintains ideal CPE conditions even when transporting through millions of GNPs with chord lengths of only a few nanometres.

The electron Fano cavity test ${ }^{72}$ is chosen for this work over a more traditional approach of a regenerative photon simulation ${ }^{74}$ for efficiency gains. The cell geometries tested are very small and complex (relative to a simple cavity) and the $<0.01 \%$ uncertainties on calculations are very computationally intensive. The electron Fano cavity test allows for the use of the recursive boundary conditions in EGSnrc, which (with the geometry specifications in section 2.2.3) ensure that $3-27 \%$ of the total simulation volume is occupied by the cell; this fraction could be further increased for more efficiency for each different cell size (if required). The electron Fano cavity test results agree with the $0.1 \%$ threshold found for EGSnrc using the more traditional (regenerative photon) Fano test on ionization chambers ${ }^{74,75}$ (indepen- 
dent of electron transport step size), even lower than the $0.3 \%$ threshold in Geant4 ${ }^{85}$ (step size parameters: dRoverRange $=0.01$, finalRange $=0.1$ ) and 0.2-0.4\% threshold for PENE$\mathrm{LOPE}^{83}$ (step parameters: $C 1=\mathrm{C} 2=0.02, W C C=0.002 \mathrm{E}_{0}$ and $W C R=0.0002 * \mathrm{E}_{0}$ for initial energy $\left.\mathrm{E}_{0}\right)$. A recent study by Lee et $a l^{92}$ using the electron Fano cavity test on ionization chambers for the purposes of magnetic field testing found that the electron Fano cavity test passed to the same thresholds as above for Geant4, PENELOPE, and EGSnrc as the regenerative photon tests when the magnetic field was set to zero.

\subsection{Conclusion}

New lattice geometries were introduced into the egspp geometry package of EGSnrc, along with multi-region scoring modifications to egs_chamber. Often used as the standard in external beam metrology ${ }^{93}$ EGSnrc, with the above changes, was tested through cross-validation with other codes. EGSnrc results scoring cell dose and dose ratios to microcavities containing GNPs agreed with independent calculations in Geant4-DNA ${ }^{70}$ and PENELOPE, ${ }^{53}$ respectively. The modified EGSnrc with added lattice geometries also passes the Fano cavity test for a complex geometry comprised of a cell and GNPs. Thus, EGSnrc can reliably calculate cell energy deposition and simulate radiation transport through GNPs. This reliability, along with much of EGSnrc's inherit efficiency when modelling accurate electron transport, ${ }^{94}$ makes EGSnrc an excellent choice for the investigation of GNPT and opens the door for further multiscale applications in radiotherapy. 


\section{Chapter 3}

\section{Tissue DEF in GNPT with HetMS}

\subsection{Introduction}

Section 1.6 of chapter 1 gives some examples of early work in GNPT MC simulation for which tumour-scale effects of GNPs were investigated. ${ }^{49,51,52}$ The DEFs calculated in those studies were macroscopic DEFs ${ }^{52}$ which do not directly reflect on the true DEFs to the non-gold media. As studies began incorporating more discrete models of GNPs and scoring dose to tissue around them, the scope of the simulation volumes became more limited (likely due to computational limits). There are a number of studies that used different methods to attempt to bridge the gap of investigating microdosimetry around GNPs in tumour-sized volumes, but often the assumptions used led to ignoring many of the required considerations of full scale GNPT. ${ }^{16}$

This chapter introduces the Heterogeneous MultiScale (HetMS) model, a model combining different levels of detail (e.g., macroscopic bulk tissue with microscopic cells) in different regions of a single simulation geometry, and uses it to investigate the microscopic dose effects of GNPs on a macroscopic (tumour) length scale. Using the modified EGSnrc (tested in chapter 2), a HetMS cylinder model is used to determine tissue DEF as a function of 
depth for various gold concentrations and source energies, investigating small $(10 \mathrm{~nm})$ and large $(50 \mathrm{~nm})$ radius GNPs. A sphere model based on work proposed by Sinha et al ${ }^{33}$ is simulated and DEF is compared with results in the work of Ngwa et al using a (non-MC) analytic technique ${ }^{64-66}$ and contextualized using the cylinder results.

\subsection{Methods}

\subsubsection{Heterogeneous multiscale modelling}

Simulations in this chapter are performed using the HetMS model which, herein, is a simple combination of distinct geometric models employed on two different scales. The HetMS phantoms are composed of single region gold-tissue mixture volume for fast transport embedded with microscopic cavities in which GNPs are discretely modelled using a cubic lattice inserted at points of interest to determine the DEF to only tissue. Russian roulette with a factor of 64 ( 1 in 64 chance of survival) is applied to any electrons that are at a distance from any scoring volume (all microscopic scoring volumes for HetMS and homogeneous scoring volumes otherwise) larger than their average range under the continuous slowing down approximation. This variance reduction technique reduces the time spent simulating electrons far from scoring regions by a factor of 64 . EGSnrc transport parameters are the same as those used in chapter 2 .

\subsubsection{Parallel beam of monoenergetic photons incident on cylinder}

A parallel beam (circular cross section, $1 \mathrm{~cm}$ radius) of monoenergetic photons with energies of $20,30,50,90$, and $200 \mathrm{keV}$, as well as $1 \mathrm{MeV}$ is incident on cylindrical phantoms $(1 \mathrm{~cm}$ radius, $3 \mathrm{~cm}$ long), depicted in figure 3.1. In the HetMS model, the cylinder is comprised of a homogeneous mixture of ICRU four-component tissue (10.1\% hydrogen, $11.1 \%$ carbon, 
$2.6 \%$ nitrogen, and $76.2 \%$ oxygen by $\left.\operatorname{mass}^{20}\right)$ and gold, containing smaller cylinders (100 $\mu \mathrm{m}$ radius, $100 \mu \mathrm{m}$ long) comprised of a lattice of discretely-modelled spherical GNPs within (pure) ICRU tissue (figure 3.1a). A total of 119 smaller cylinders evenly-spaced along the central axis of the cylindrical phantom are simultaneously modelled and energy deposited within the tissue (not GNPs) is scored. The lattices within the microscopic scoring cavities are rotated to avoid aligning with the incoming photon beam, as described in chapter 2 section 2.2.1. A radius and thickness of a $100 \mu \mathrm{m}$ is chosen for the microscopic scoring cavity, as the $100 \mu \mathrm{m}$ is orders of magnitude smaller than the cm-scale of the macroscopic phantom (ensuring macroscopic fluence does not change drastically over the cavity) but still large enough to be able to calculate dose with low uncertainties within reasonable time frames for the cavities farthest from the source.

For comparison, a fully homogeneous cylinder is modelled in which all regions are a mixture of tissue and gold and dose is scored therein (figure 3.1b). Simulations are then repeated with a tissue-only phantom to provide the denominators for computation of DEFs (figure 3.1c). Simulations are performed for concentrations of 5, 10 and $20 \mathrm{mg} / \mathrm{g}$ (common concentrations used in the literature), and GNP diameters of 20 and $100 \mathrm{~nm}$. Results for the HetMS model (figure 3.1a) are compared to those from simulations of the cylinder comprised of tissue entirely filled with a GNP lattice (for a subset of source energies and gold concentrations) for further validation, as well as characterization of simulation efficiencies. Most scenarios are comprised of $10^{9}$ histories simulations, though some simulation used as many as $4 \times 10^{9}$ histories when the gold in the scatter media caused photon starving far from the source. 


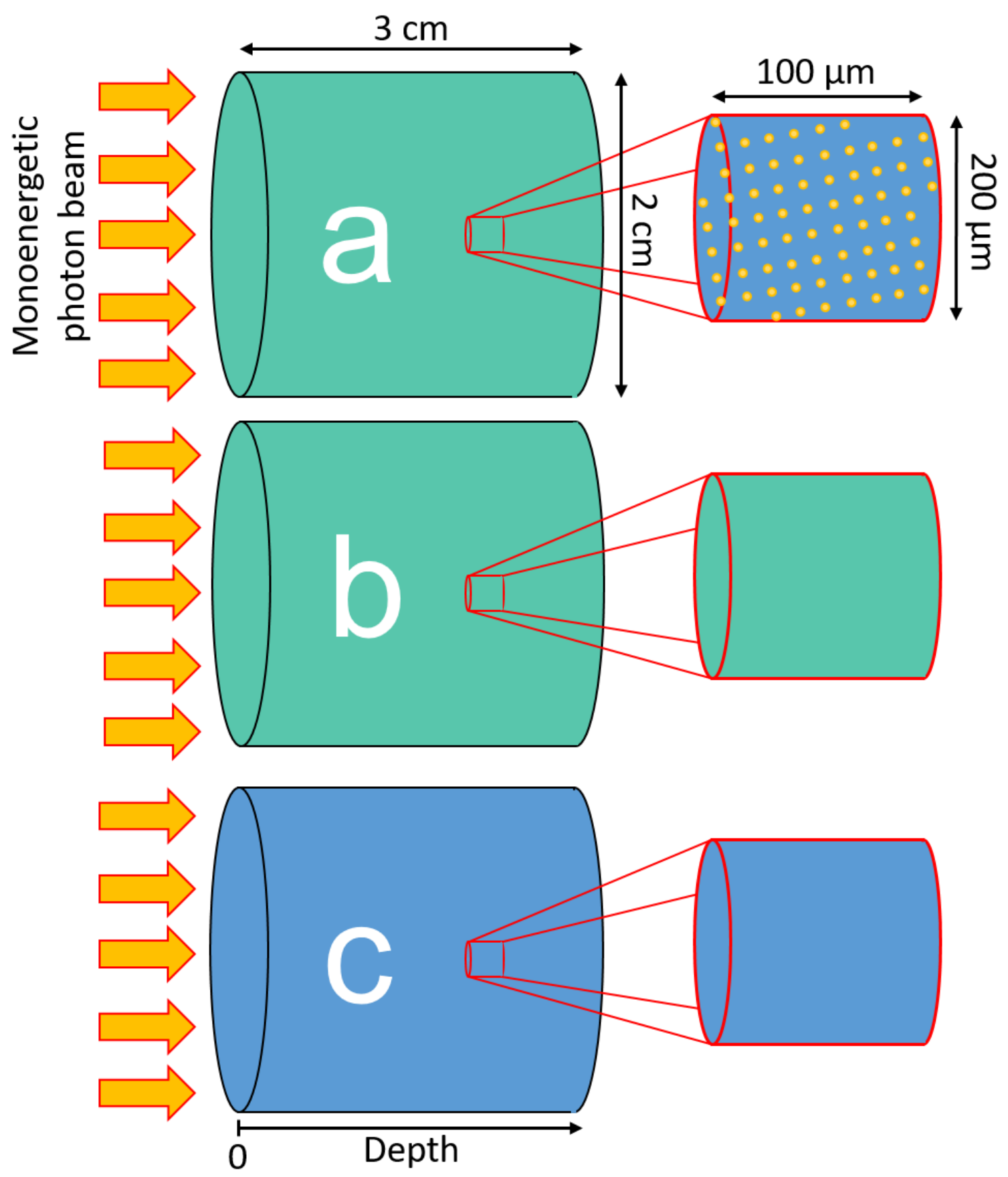

Figure 3.1: Schematic diagrams for cylindrical phantom simulations: (a) HetMS model with homogeneous gold-tissue phantom with 119 smaller cylinders consisting of GNPs embedded in tissue placed at even intervals along the central axis; (b) corresponding fully homogeneous (goldtissue mixture) phantom; and (c) pure tissue phantom. 


\subsubsection{Brachytherapy source in a sphere with varying gold concen- tration}

A more realistic GNPT scenario, from the work of Sinha et al, ${ }^{33}$ is simulated using the HetMS framework. The proposed treatment considers adding a polymer film embedded with GNPs coated on the inter-seed spacers used in prostate permanent implant brachytherapy; the film would dissolve over time in the patient, releasing GNPs which diffuse outward to create a significant concentration of gold in the treatment volume. Sinha et al calculated DEFs using a previously-developed analytical calculation approach: ${ }^{64}$ calculations were performed at different radii within a water sphere containing an isotropic point source $\left({ }^{125} \mathrm{I},{ }^{103} \mathrm{Pd}\right.$ and ${ }^{131} \mathrm{Cs}$ ) at its centre. Assuming GNPs steadily released from the sphere centre and, using Fick's second law of diffusion, ${ }^{95}$ GNP concentration was determined as a function of time and radial position within the sphere, enabling computation of DEFs. No particular localization of GNPs within tumour cells was assumed, i.e., GNPs randomly arranged within a region of a given concentration.

In the HetMS model simulation of the above scenario, a $2.5 \mathrm{~cm}$ radius sphere divided into $0.5 \mathrm{~mm}$ shells each containing a homogeneous mixture of gold and water with concentrations based on the diffusion of GNPs from the centre is modelled (figure 3.2), following the same procedure as Sinha et al. ${ }^{33}$ The 50 shells are a discrete approximation of the continuous decrease in concentration expected. At the centre of each homogeneous shell, a $100 \mu \mathrm{m}$ thick spherical shell comprised of $20 \mathrm{~nm}$ diameter GNPs in a lattice embedded in water is modelled, and dose is scored in water within these regions. An isotropic point source at the centre of the sphere is used assuming the spectra leaving the following seed models: ${ }^{96}$ OncoSeed $6711^{97,98}\left({ }^{125} \mathrm{I}\right)$, IsoRay CS1 ${ }^{99,100}\left({ }^{131} \mathrm{Cs}\right)$, and TheraSeed $200^{100,101}\left({ }^{103} \mathrm{Pd}\right)$. Simulations are repeated with an all-water geometry (no gold) to provide the denominators to compute DEFs. $10^{10}$ histories are used for all spherical HetMS simulations. 

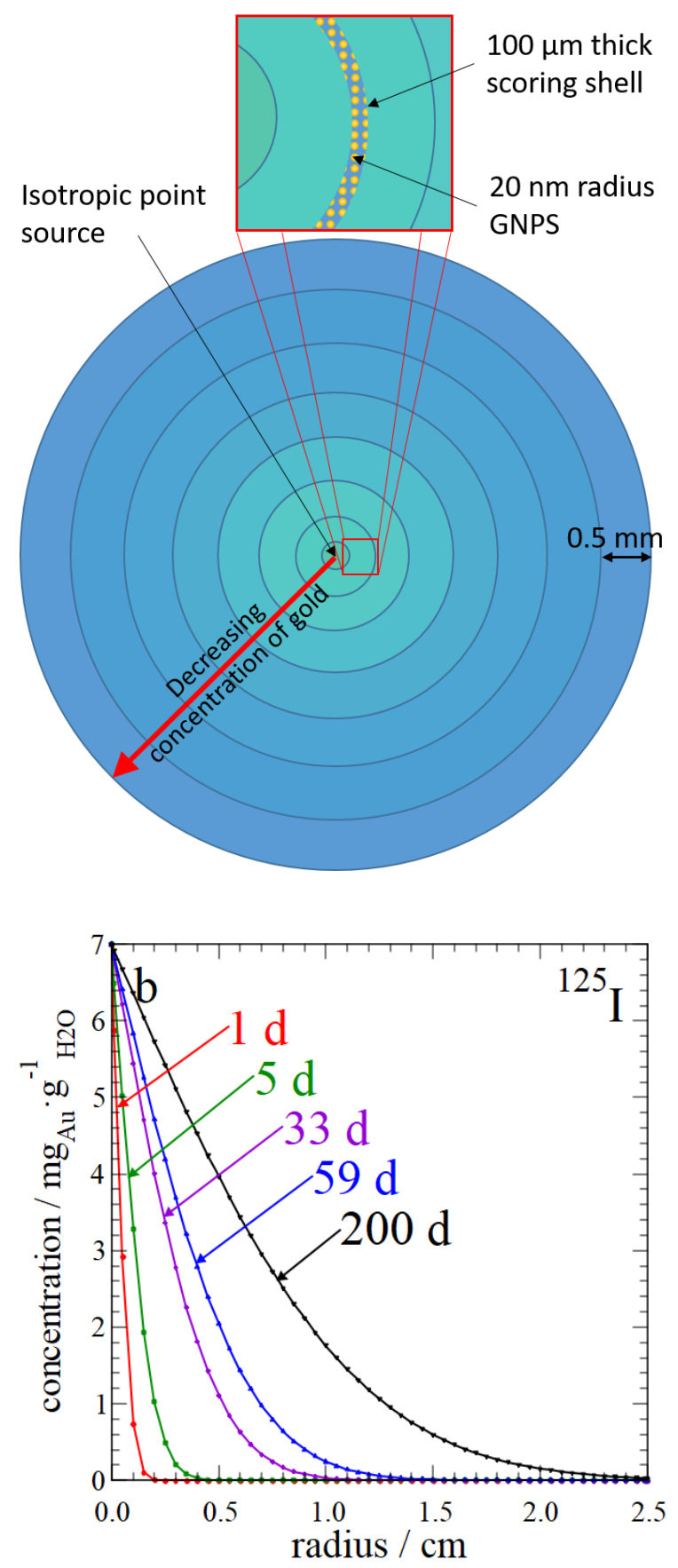

Figure 3.2: (a) Schematic cross-sectional diagram of the sphere with GNPs released from its centre; homogeneous water-gold mixtures are modelled in spherical shells $0.5 \mathrm{~mm}$ thick. A $100 \mu \mathrm{m}$ thick spherical shell consisting of discretely-modelled GNPs in water (shown above) is embedded in each shell. (b) Gold concentrations within the sphere as a function of radius for different times after implantation assuming $7 \mathrm{mg} \mathrm{Au} / \mathrm{g}$ water at the centre and following Fick's second law of diffusion. 


\subsection{Results}

\subsubsection{Parallel beam of monoenergetic photons incident on cylinder}

The results of the cylinder simulations for $20 \mathrm{~nm}$ diameter GNPs are summarized in figure 3.3. Focusing first on photon source energies below $200 \mathrm{keV}$, DEFs for all concentrations are most substantial near the surface of the phantom, ranging from 1.6 to 4.2, and decrease with depth. DEFs decrease more rapidly with increasing depth in the $20 \mathrm{mg} / \mathrm{g}$ case: for the $50 \mathrm{keV}$ source, DEFs decrease from 4 to 2.5 over the $3 \mathrm{~cm}$ length of the phantom, while DEFs for the $20 \mathrm{keV}$ source are near 3 at the surface and decrease nearly to zero. DEFs decrease below unity for some gold concentrations and source energies: for $5 \mathrm{mg} / \mathrm{g}$ and a $20 \mathrm{keV}$ source, there are DEFs $<1$ for depths of $1 \mathrm{~cm}$ or more; for 10 and $20 \mathrm{mg} / \mathrm{g}$, DEFs drop below unity within the $3 \mathrm{~cm}$ long phantom for a $30 \mathrm{keV}$ beam as well. These phenomena arise due to competing effects of enhanced local energy deposition (due to photoelectric interactions in local GNP) and decreasing fluence with depth (due to the presence of gold between the front of the cylinder and the scoring depth, i.e., within the scatter media), and are expected on the basis of energy conservation. When the effects of the decreasing fluence exceed local energy deposition gains, dose scored within the HetMS model is lower than that in the pure tissue phantom (no gold), resulting in DEFs below unity, i.e., dose decreases not enhancements, making DEF a misnomer. Despite this, the dose ratio will continue to be referred to as a DEF for the purposes of continuity.

As source energy increases, the relative importance of photoelectric events decreases resulting in DEFs nearer unity and less variation in DEFs with depth. For $200 \mathrm{keV}$ photons, DEFs are approximately constant with depth within the $3 \mathrm{~cm}$ phantom, at a value near 1.05

for $5 \mathrm{mg} / \mathrm{g}$ and 1.40 for $20 \mathrm{mg} / \mathrm{g}$. For the $1 \mathrm{MeV}$ beam, incoherent (Compton) interactions dominate resulting in DEFs near unity for the length of the cylinder - i.e., no significant dose enhancement. 

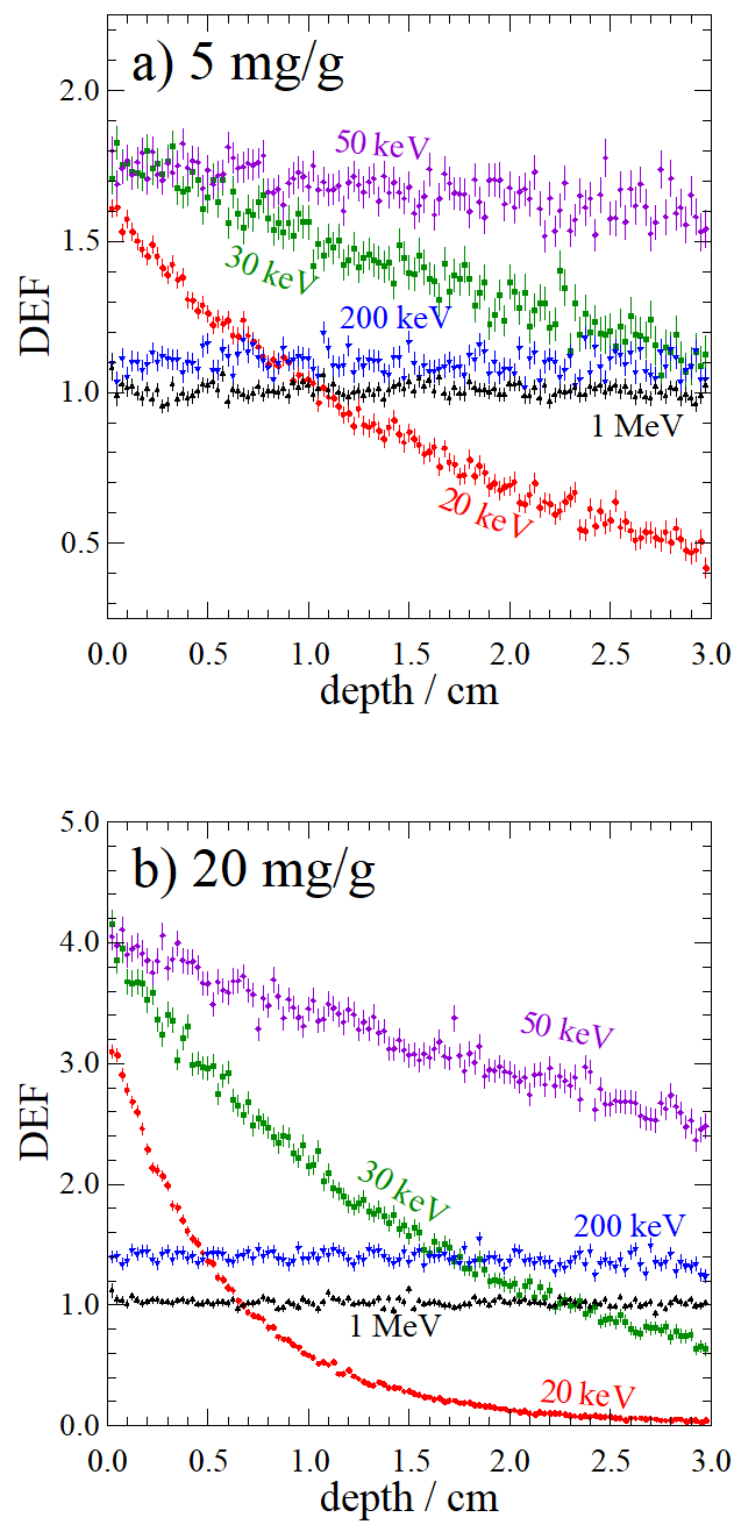

Figure 3.3: DEFs versus depth within the cylindrical phantom (figure 3.1) with concentrations of (a) 5 and (b) $20 \mathrm{mg}$ of Au per g of tissue for $20 \mathrm{~nm}$ diameter GNPs with incident photon source energies of $20 \mathrm{keV}$ to $1 \mathrm{MeV}$. DEFs for $90 \mathrm{keV}$ which show smaller magnitude but similar trends to the results for $50 \mathrm{keV}$ are omitted for clarity. Results for simulations with a concentration of $10 \mathrm{mg} / \mathrm{g}$ (omitted) exhibit similar trends to 5 and $20 \mathrm{mg} / \mathrm{g}$. 
DEFs computed with 20 or $100 \mathrm{~nm}$ diameter GNPs in scoring regions (HetMS model) along with the homogenized tissue-gold mixture are summarized in Figure 3.4 for the $20 \mathrm{keV}$ photon source. In general, DEFs within the homogeneous model (qualitatively) follow the same trends as those in the HetMS model, showing competition between attenuation of fluence and enhanced energy deposition. However, for all gold concentrations and GNP diameters, DEFs computed within the homogeneous model overestimate those for the HetMS model: discrepancies are as large as $20 \%$ for the $20 \mathrm{keV}$ beam and vary with depth. As source energy increases, discrepancies between DEFs computed with the HetMS and homogenized models decrease (results not shown; see also dose ratios presented in table 2.1, chapter 2). The overestimation observed for DEFs computed in the homogenized model stems from the inclusion of energy deposited within gold into the total 'enhanced' dose, while it is only dose to tissue (not gold) that is relevant for GNPT. ${ }^{57,78}$ In the HetMS simulations with GNPs modelled discretely and dose in tissue scored, photoelectrons generated in the gold may deposit all their energy in the gold or they must travel some distance (and deposit energy) before entering tissue. By energy conservation, there is then less dose in the numerator for the computation of DEFs. Comparing HetMS model results for 20 and $100 \mathrm{~nm}$ diameter GNPs but same total gold concentration, DEFs are consistently higher with smaller GNPs for lower source energies (20 and $30 \mathrm{keV}$ ) but do not vary significantly at higher energies. Sensitivity to GNP size stems from the re-absorption of electrons generated within GNPs which is more important for larger GNPs. ${ }^{57,78}$

All results presented with the HetMS model thus far might have been carried out in a phantom with each GNP modelled discretely, rather than combining models with different features on different length scales, as done for the HetMS model. We carried out a subset of simulations in a cylindrical phantom comprised of ICRU tissue with a lattice of GNPs spanning the entire phantom, and results agreed with those computed within the HetMS model with GNPs only modelled in subvolumes (figure 3.1) within statistical uncertainties. The major difference between the two sets of simulations was computing time or, equivalently, 


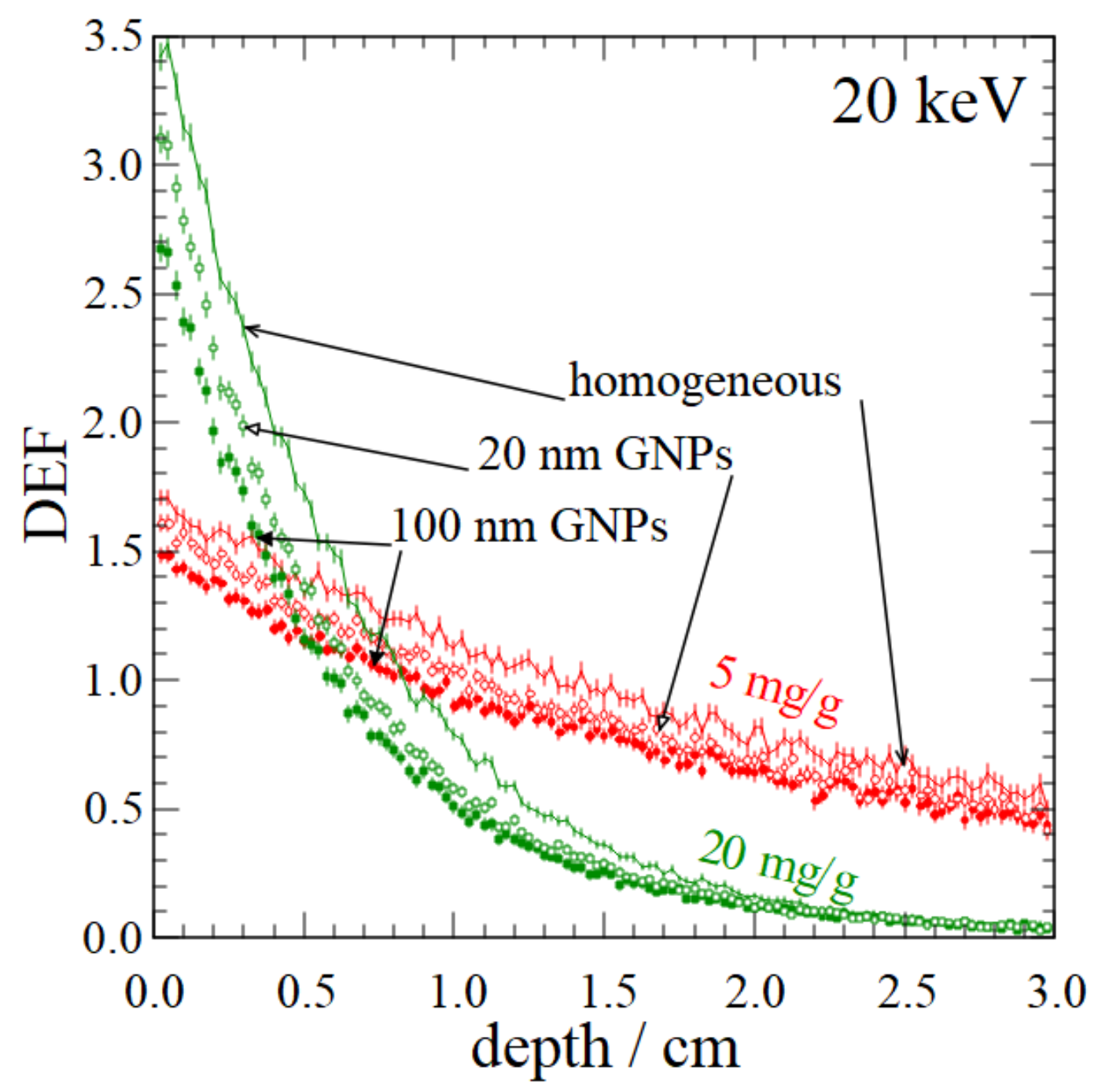

Figure 3.4: DEFs versus depth within the cylindrical phantom (figure 3.1) with concentrations of 5 and $20 \mathrm{mg} / \mathrm{g}$ for 20 and $100 \mathrm{~nm}$ diameter GNPs with $20 \mathrm{keV}$ incident photons. DEFs computed for the 30 and $50 \mathrm{keV}$ source energies show similar trends to the $20 \mathrm{keV}$ case but are omitted for clarity. 
efficiency:

$$
\epsilon=\frac{1}{s^{2} t}
$$

where $t$ is the simulation time and $s$ is the average percent uncertainty on doses in the 119 scoring regions (spanning the $3 \mathrm{~cm}$ long cylindrical phantom). Table 3.1 demonstrates considerable efficiency gains with the HetMS model compared with having a lattice filling the entire volume of interest, with efficiencies enhanced by factors of $24(20 \mathrm{mg} / \mathrm{g} ; 20 \mathrm{keV})$ to 122 (5 mg/g; $50 \mathrm{keV})$; HetMS simulations have lower efficiencies than the corresponding (inaccurate) homogeneous models by $15 \%(5 \mathrm{mg} / \mathrm{g} ; 20 \mathrm{keV})$ to $31 \%(20 \mathrm{mg} / \mathrm{g} ; 20 \mathrm{keV})$. Efficiencies vary with source energy and gold concentration, e.g., the lower efficiency of the $20 \mathrm{keV}$ photons and gold concentration $20 \mathrm{mg} / \mathrm{g}$ reflects larger statistical uncertainties at depth in the cylinder (due to considerable attenuation of these relatively low-energy photons with the higher concentration of gold). As the results of the full lattice simulation agreed with the HetMS model, it is evident that full microscopic detail throughout the phantom is not required to measure DEF. Thus, the same microscopic dose metrics can be measured at potentially one hundredth of the time using HetMS.

Simulation times (on a single Intel Xeon 5160 core) may be deduced from the results in table 3.1: for the concentrations and source energies considered therein, HetMS simulation times for $s=2 \%$ average uncertainty range from 0.12 years $(5 \mathrm{mg} / \mathrm{g} ; 50 \mathrm{keV})$ to 0.91 years (20 mg/g; $20 \mathrm{keV})$ while full lattice simulation times range from 3.9 years $(5 \mathrm{mg} / \mathrm{g} ; 20 \mathrm{keV})$ to 22 years $(20 \mathrm{mg} / \mathrm{g} ; 20 \mathrm{keV})$. The results presented in Figs. 3.3 and 3.4 are from simulations of $>10^{10}$ histories, with number of histories adjusted to achieve the relatively small error bars (depending on source energy and gold concentration). We have not included the times for these particular calculations because simulations were run on multi-core clusters with CPUs of varying speeds, and hence these calculation times (or efficiencies) are not representative measures. 
Table 3.1: Example efficiencies (equation 3.1) for HetMS simulations in the cylindrical phantom (figure 3.1a), with efficiencies relative to the tissue cylinder entirely filled with a lattice ("HetMS/Lattice") and to the homogeneous gold-tissue mixture cylinder (figure 3.1b; "HetM$\left.\mathrm{S} / \mathrm{Homog}^{\prime}\right)$. Estimates of efficiencies are based on calculation times for simulation of $3.6 \times 10^{6}$ histories on a single thread Intel Xeon $5160(3.00 \mathrm{GHz})$ core. The time in h to achieve $s=1 \%$ is $1 / \epsilon$.

\begin{tabular}{ccccc}
\hline \hline Concentration & Energy & Efficiency & \multicolumn{2}{c}{ Relative efficiency } \\
\cline { 4 - 5 }$\left(\mathrm{mg}_{\text {Au }} / \mathrm{g}_{\text {tissue }}\right)$ & $(\mathrm{keV})$ & HetMS $\left(\mathrm{h}^{-1}\right)$ & HetMS/Lattice & HetMS/Homog \\
\hline 5 & 20 & $2.2 \times 10^{-4}$ & 29 & 0.85 \\
& 50 & $2.4 \times 10^{-4}$ & 122 & 0.69 \\
20 & 20 & $3.1 \times 10^{-5}$ & 24 & 0.73 \\
& 50 & $2.1 \times 10^{-4}$ & 89 & 0.72 \\
\hline \hline
\end{tabular}

\subsubsection{Brachytherapy source in a sphere with varying gold concen- tration}

DEFs as a function of radius computed within the HetMS model for the spherical phantom with varying gold concentration (figure 3.2 ) are shown in figure $3.5 \mathrm{a}$ for ${ }^{125} \mathrm{I}$ at different times. Near the source, the DEFs range from 1.4 after 1 day to near 1.9 after 33 days or more. DEFs go to unity at small radii for the 1 and 5 day cases, whereas decreases in DEFs to unity happen at larger radii later in the treatment (33 days or more). For each treatment time considered, DEFs drop below one at some radius, e.g., $0.35 \mathrm{~cm}$ for 5 days, $0.7 \mathrm{~cm}$ for 33 days, and $1.2 \mathrm{~cm}$ for 200 days. As observed in the cylindrical phantom computations (section 3.3.1), DEFs $<1$ correspond to a dose decrease due to decreasing fluence resulting from gold between the source and scoring regions. For the current spherical geometry involving varying gold concentrations (figure 3.2b), dose decreases are observed in regions where there is a non-zero concentration of gold, as well as at larger radii where effectively no GNPs have diffused. Similar trends are observed for the ${ }^{131} \mathrm{Cs}$ and ${ }^{103} \mathrm{Pd}$ sources (figure 3.5b), however, exact DEF values depend on radionuclide source spectrum, with lower (higher) DEFs for ${ }^{103} \mathrm{Pd}$ $\left({ }^{131} \mathrm{Cs}\right)$ compared to ${ }^{125} \mathrm{I}$.

DEFs from Sinha et $a l^{33}$ are presented alongside the HetMS results in figure 3.5. While 

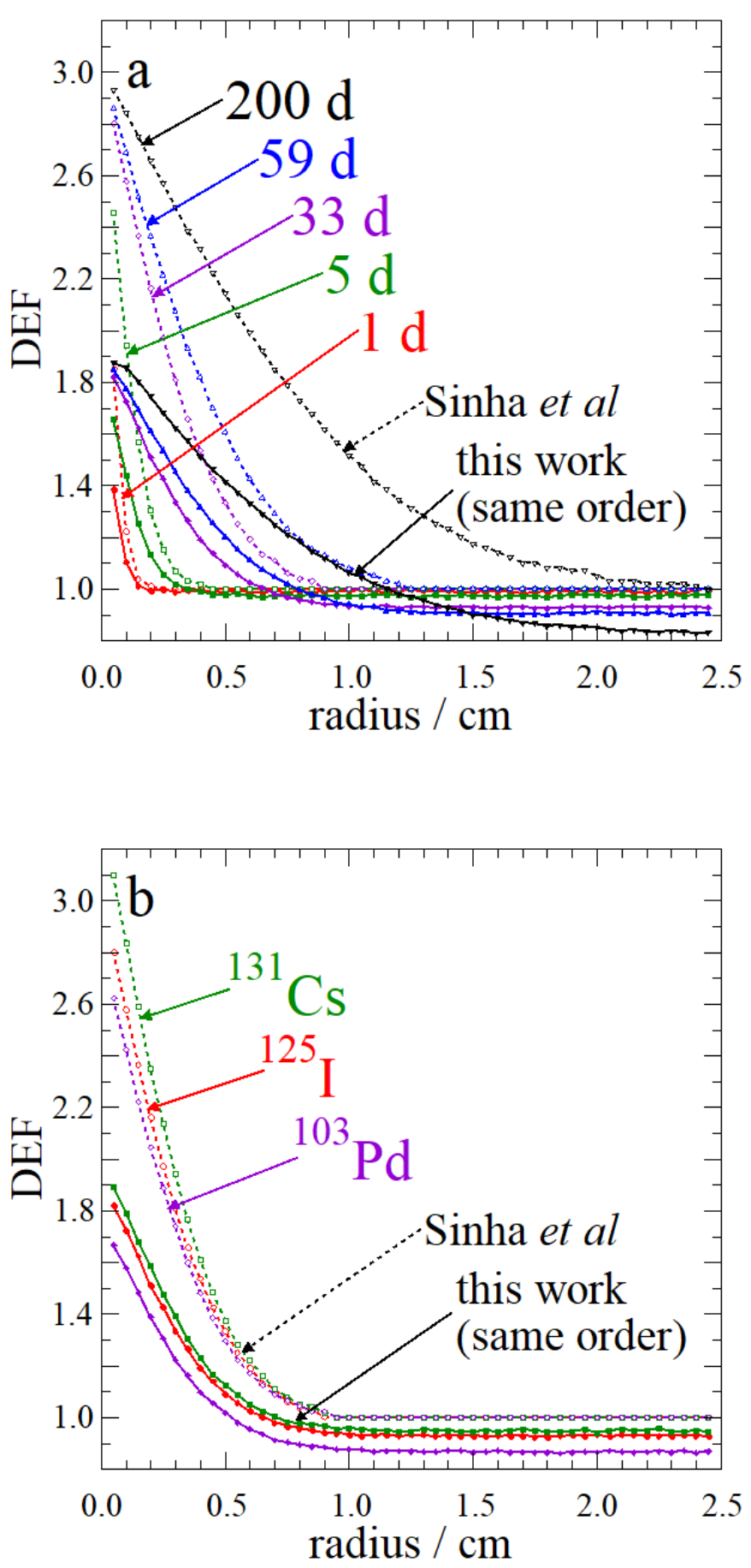

Figure 3.5: (a) DEF versus radius within the spherical phantom (figure 3.2) at different times during treatment with ${ }^{125} \mathrm{I}$. (b) Comparison of DEF versus radius for different radionuclides after 33 days. Both panels present results of the current work (closed symbols and solid lines; error bars for statistical uncertainties are often too small to see) as well as data extracted from Sinha et $a l^{33}$ using plot digitization (open symbols and dotted lines). 
both sets of DEFs follow the same trends, they differ considerably: DEFs computed by Sinha et al are consistently higher for all radii and times, and for all three radionuclides. For early times in the treatment ( 1 and 5 days), 40\% discrepancies are observed near the source, but agreement is better near a radius of $5 \mathrm{~mm}$ where little gold has diffused. For later times (33 days or more), differences range from 5 to $50 \%$. Most notably, DEFs computed by Sinha et al never drop below one - all DEFs converge to unity with increasing radius whereas the effects of increased fluence perturbation with radius (due to gold), seen in HetMS results in figure 3.5, are not apparent.

\subsection{Discussion}

The cylindrical and spherical phantom examples illustrate the main ideas of the HetMS model for GNPT: distinct models are employed on different length scales in order to capture relevant physics effects within a single, relatively efficient, simulation. While these ideas are generalizable to consider many issues in GNPT using the HetMS approach (more below), the current work focuses on relatively simple HetMS models involving homogenized tissue-gold mixtures (or pure tissue) in the bulk of phantoms (possibly with varying gold concentration) with 50 or 119 microscopic regions containing discretely-modelled GNPs in tissue. Employing the homogenized, tissue-gold mixture (or pure tissue) within the bulk of the phantom enables realistic representation of scatter conditions and determination of fluence. The discrete modelling of GNPs within smaller $(\sim \mu \mathrm{m})$ regions enables scoring of energy deposition within the tissue in which GNPs are embedded.

The HetMS approach enables considerably more efficient simulations than otherwise possible with discrete modelling of GNPs throughout the entire GNP-containing volume (as

done previously $\left.{ }^{61,77-81}\right)$. We observed efficiency gains of factors of up to 122 within the cylindrical phantom (table 3.1), with efficiency gains varying with source energy and gold 
concentration. In general, efficiency gains would depend on the details of the geometry considered, e.g., phantom size; number, size, and geometries of scoring regions; GNP concentration, source energy, and so on. In certain circumstances, discrete modelling of each GNP within the macroscopic volume would be impossible with available computer resources (memory, computing time). Indeed, calculation times for the simulations presented herein would have been prohibitively long without the HetMS approach - performing the current study using discrete modelling of every GNP within the phantoms would have been computationally infeasible. All of the cylindrical phantom simulations were completed in less time than it would take to run the cylindrical simulation modelling discrete GNPs everywhere for only an energy of $20 \mathrm{keV}$ and concentration of $20 \mathrm{mg} / \mathrm{g}$.

As discussed in chapter 2, verification (beyond cross-validation) of computational models used to determine DEF can be quite difficult. Additional tests were performed in the current chapter to ensure self-consistency. The use of $1 \mathrm{keV}$ energy transport cutoffs for photons and electrons was investigated by repeating simulations with these cutoffs increasing in $125 \mathrm{eV}$ increments up to $2 \mathrm{keV}$ (i.e., $1.000 \mathrm{keV}, 1.125 \mathrm{keV}, 1.250 \mathrm{keV}, \ldots, 2.000 \mathrm{keV}$ ); results agreed within statistical uncertainties up to $1.75 \mathrm{keV}$. Several results in figure 3.3 were replicated using $10 \mu \mathrm{m}$ long scoring cylinders instead of $100 \mu \mathrm{m}$ cylinders, hence demonstrating insensitivity to variations in the $\mu \mathrm{m}$ scoring region dimensions

DEFs computed for the cylindrical phantom scenario (section 3.3.1) demonstrate the competing effects of enhanced local energy deposition and decreasing fluence with depth in the phantom. While dose enhancements are observed for many source energies, DEFs change considerably with depth: DEFs $<1$ are observed for lower energy sources (20, $30 \mathrm{keV}$ ) within the phantom. These dose decreases have the potential to create cold spots in a treatment volume and must be accounted for in any algorithm for GNPT dosimetry or treatment planning.

The results of our cylinder simulations are generally comparable with those presented 
in other published works. For example, the magnitudes of DEFs observed at the front of the cylindrical phantom for 20 and $30 \mathrm{keV}$ sources (figure 3.3) are generally in agreement with those predicted by Roeske et al for ${ }^{103} \mathrm{Pd}$ and ${ }^{125} \mathrm{I}$ using analytic calculations. ${ }^{102}$ Results of some other works show gradients in DEFs over $\sim \mathrm{cm}$ length scales (although often not dose decreases, DEFs < 1), e.g., MC models employing a $(1 \mathrm{~cm})^{3}$ lattice of GNPs and considering kilovoltage photon/brachytherapy sources, ${ }^{78,80}$ as well as MC models involving homogenized tissue-gold mixtures (no discrete modelling of GNPs) irradiated by ${ }^{125} \mathrm{I}, 50 \mathrm{kVp}$ x ray, and ${ }^{169} \mathrm{Yb}$ sources. ${ }^{52}$ In contrast, results of the similar work of Mesbahi et al (who modelled a $(1 \mathrm{~cm})^{3}$ region containing a lattice of GNPs (of varying diameter; concentrations of 7 and $18 \mathrm{mg} / \mathrm{g}$ within a larger phantom) do not have a downward trend in DEFs with depth. This is likely due to the relatively low resolution of their dose scoring grid, the shorter distance $(1 \mathrm{~cm})$ they considered within the region containing GNPs (along with the magnitude of statistical uncertainties), or the fact that the lattice had an axis aligned with the parallel beam incident on the phantom (see figure 1 in Mesbahi et $a l^{77}$ ). Within the context of tumour dose enhancement using modified megavoltage beams and gadolinium or iodine contrast media (not GNPT), Robar et al noted decreasing trends in dose enhancement over centimetre length scales. ${ }^{103}$

With the potential for considerable DEFs at kilovoltage energies, previous studies have investigated DEFs for brachytherapy involving GNPs (see, e.g., refs. ${ }^{52,54,57,78,80,88}$ ). Recent works considered new avenues for GNP delivery within the context of accelerated partial breast irradiation with an electronic brachytherapy source ${ }^{67}$ and prostate brachytherapy ${ }^{33}$ with GNPs diffusing within the treatment volume. Our spherical phantom simulations considered the latter scenario, with considerable discrepancies (up to 50\%) observed between the published DEFs computed by Sinha et al and those from our HetMS model (figure 3.5).

Sinha et al indicate $10 \%$ uncertainties on their DEFs, ${ }^{33}$ citing another work dealing with dose enhancement to endothelial cells via megavoltage $\mathrm{x}$ rays and GNPs, ${ }^{104}$ however, 
it is unclear where the figure of $10 \%$ originates given the different considerations for the kilovoltage sources in the brachytherapy context. Uncertainties of $10 \%$ do not account for the observed discrepancies in figure 3.5. While seed models and spectra of Sinha et al were unspecified, different spectra do not explain discrepancies as we found relatively small variations in DEFs computed assuming different spectra of the same radionuclide. Sinha et al further note that their analytic approach ${ }^{64}$ to compute DEFs has been validated against MC results in previous publications. ${ }^{33}$ However, there are many approximations in this analytic calculation that are questionable in the current context, e.g., ignoring the effects of scatter and attenuation of primary photons due to gold. These approximations may result in the considerable discrepancies between the DEFs computed via the analytic approach and the

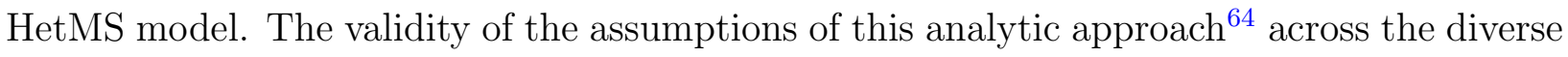
published applications ${ }^{33,65,67,104}$ is doubtful.

\subsection{Conclusion}

This chapter presents a simultaneous multiscale approach, described as the Heterogeneous MultiScale (HetMS) model, accounting for both macroscopic and microscopic considerations in a single simulation to accurately model the effects of GNPs within the radiotherapy context. We have demonstrated the HetMS approach within the simple model of a cylindrical phantom consisting of homogeneous gold-tissue mixture in which microscopic regions of discretely-modelled GNPs dispersed in tissue are embedded, as well as within a recentlyproposed prostate brachytherapy scenario involving GNPs diffusing from seed spacers. The HetMS approach enables consideration of both scatter conditions and changes in fluence over the averaged gold-tissue medium, as well as scoring in tissue containing GNPs but not within the GNPs themselves. Compared to discretely-modelling GNPs throughout the gold-containing volume, efficiency gains of factors of up to 122 were observed with HetMS simulations, enabling the calculations within the diverse scenarios considered herein with 
available computing power. Both sets of example calculations demonstrate considerable and varying dependence of DEFs with depth in tissue, gold concentration, GNP size, and source energy. These results, as well as observed discrepancies with DEFs computed using a broadlyapplied analytic technique involving considerable approximations, emphasize the importance of HetMS modelling for lower source energies, e.g., brachytherapy or orthovoltage treatments using GNPs. The HetMS MC simulations may be extended in various ways, offering new avenues for GNPT research. 


\section{Chapter 4}

\section{Single-cell DEF in GNPT}

\subsection{Introduction}

In chapter 3, HetMS simulations were performed to compute DEFs over a tumour-sized volume. The microscopic scoring cavities used for the HetMS simulations consisted of GNPs dispersed evenly (in a lattice) in tissue. As the primary focus of that chapter was on the macroscopic effects of filling a large scatter volume with gold, many microscopic considerations were ignored. A more realistic (biological) approach to modelling GNPs should consider cell models and how GNPs aggregate within them. There are many biological aspects when delivering GNPs to cells ${ }^{38,39}$ that need to be accounted for, such as variable GNP uptake ${ }^{40}$ GNPs aggregating in different configurations within a cell, ${ }^{41}$ and cells (and nuclei) differing in size. ${ }^{105}$ Though there have been Monte Carlo studies which computed DEFs on

a cellular scale $58,60-62,106,107$ they were typically investigated over a limited parameter space. Simulations were often limited to a few (or only one) choices for incident energy, cell size, GNP configurations and gold concentration parameters.

This chapter investigates the variation in cellular DEFs for a large number of parameters. Different approaches are investigated for modelling gold within a cell: a contiguous pure 
gold region, a homogeneous mixture of tissue and gold, or discrete GNP models. Cellular nucleus and cytoplasm DEFs are computed using discretely modelled GNPs in one of three configurations in a cell: GNPs distributed evenly across the (outside) surface of the nucleus, GNPs aggregated into a single compartment in the cytoplasm, or GNPs aggregated into four compartments instead. These GNP configurations could lead to significant differences in nucleus and cytoplasm DEFs, and should be important considerations for GNPT. For the three different configurations, cell (nucleus and cytoplasm) DEFs are computed for nine different cell sizes, for various source energies and gold concentrations.

\subsection{Methods}

The media used in the MC simulations in this chapter are gold, ICRU four-component tissue (10.1\% hydrogen, $11.1 \%$ carbon, $2.6 \%$ nitrogen, and $76.2 \%$ oxygen by mass ${ }^{20}$ ) or a homogeneous mixture of the two. Spherical cells are modelled as a central sphere representing the nucleus and an outer spherical shell representing cytoplasm. A reference cell $\left(r_{\text {cell }}, r_{\text {nuc }}\right)$ $=(7.35,5) \mathrm{\mu m}$ is used for all cell models in this chapter, ${ }^{105}$ except in section 4.2 .2 where different radii are investigated. Cells are placed in the centre of a $100 \mu \mathrm{m}$ radius spherical phantom containing only tissue. Thus, the gold within the cell is the only gold in the simulation. For all simulations of cells with GNPs, an all-tissue cell with no GNPs model is also simulated to compute nucleus and cytoplasm dose. The no-gold cell doses are then used to compute the DEF to the nucleus and cytoplasm (nDEF and cDEF, respectively; n,cDEF, collectively).

Monoenergetic photons of energies ranging 10-370 keV are generated isotropically within a spherical shell at the outer edge (1 nm from the outside) of the $100 \mu \mathrm{m}$ radius phantom. All simulations are run for $4 \times 10^{10}$ histories and no variance reduction techniques are used. EGSnrc transport parameters are the same as those used in chapter 2. 


\subsubsection{Modelling GNPs within a cell}

Three gold configurations are considered by defining different regions within a cell as the 'gold-containing region'. The first gold-containing region is a thin shell around the nucleus, called the perinuclear ("peri") configuration, which represents gold accumulating in the region close to the cell nucleus. ${ }^{108}$ The second configuration is a region which consists of a sphere placed with its centre equidistant to the nuclear radius and outer radius of the cell, called the one endosome ("1-endo") case, which represents GNPs aggregating in a single compartment which sits in the cytoplasm. ${ }^{109}$ The final configuration, called the four endosomes ("4-endo") case, is similar to the "1-endo" configuration, but with the volume split evenly amongst four spheres placed at the four corners of a tetrahedron around the nucleus (endosome centres are still equidistant to the nucleus and outer radius of the cell). The three different configurations are depicted in figure 4.1. The scoring volumes are either the entire nucleus or the regions of the cytoplasm that do not contain gold, to compute $\mathrm{nDEF}$ and $\mathrm{cDEF}$ respectively.

(a) peri

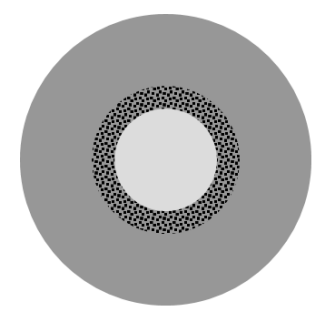

(b) 1-endo

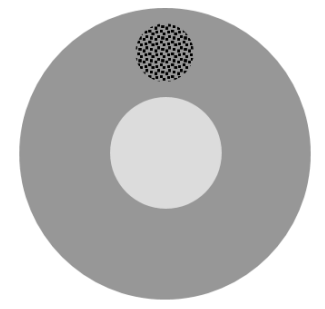

(c) 4-endo

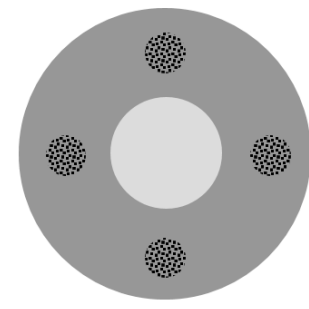

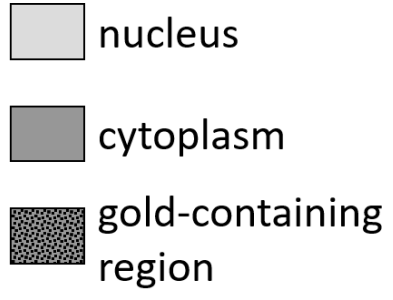

Figure 4.1: A 2D cross-sectional diagram of a cell with three different gold configurations: (a) perinuclear, (b) single endosome, and (c) four endosomes. All endosomes are translated into the same plane for visualization.

GNPs are modelled as $25 \mathrm{~nm}$ radius spheres of pure gold (when modelled explicitly). A minimum $5 \mathrm{~nm}$ thick buffer region is ensured around each gold-containing region (and each individually modelled GNP) for all cases; this buffer accounts for some of the polyethylene glycol (PEG) surface coatings, ${ }^{110}$ in which no dose is scored. The GNP number density in an endosome, where GNPs are packed as tightly as possible while allowing a minimum $5 \mathrm{~nm}$ 
distance between any two GNPs, is thus $6.547 \times 10^{15} \mathrm{GNPs} / \mathrm{cm}^{3}$. Typical gold concentrations investigated are 5, 10 and $20 \mathrm{mg} / \mathrm{g}$, as well as all integer concentrations between 4 and $24 \mathrm{mg} / \mathrm{g}$ for a subset of energies. All gold is assumed to be taken up by the cell (i.e., no gold in extracellular medium) and the total mass of gold per cell is chosen such that the average gold concentration of the overall medium remains the same. For example, for an average gold concentration $c$ (units of $\mathrm{mg} / \mathrm{g}$ ), the number of GNPs in a cell $\left(n_{\mathrm{GNP}}\right)$ must satisfy

$$
\frac{m_{\mathrm{GNP}} \cdot n_{\mathrm{GNP}} \cdot N_{\mathrm{cell}}}{\rho_{\mathrm{tissue}}}=c
$$

where $m_{\mathrm{GNP}}$ is mass of gold of a single GNP and $N_{\text {cell }}$ is number of cells per $\mathrm{cm}^{3}$ of tissue $\left(3 \times 10^{8}\right.$ for reference cell $) ;^{82} \rho_{\text {tissue }}$ is $1 \mathrm{~g} / \mathrm{cm}^{3}$ for the ICRU tissue used in this model. This equation does not account for the displaced tissue volume in the cells by the GNPs, but for all concentrations studied in this work, this volume had a negligible effect on calculated values.

Several approaches to modelling gold within a cell are investigated to ensure accurate modelling while maximizing efficiency. Figure 4.2 shows a pictographic representation of these models (figure 2.6 in chapter 2 shows a "peri" image generated with EGSnrc). The GNP-containing region is modelled using three different approaches: containing only contiguous gold, consisting of a homogeneous gold-tissue mixture which accounts for the intervening tissue, or as a hexagonal lattice; when placed in a hexagonal lattice, GNPs intersecting the boundary are modelled as only the partial volume within the gold-containing region. The contiguous gold model has been used before by Douglass et $a l^{58}$ and the explicit GNP lattice model has been used by Cai et al; ${ }^{61}$ the homogeneous mixture model is introduced as a third model that is as simple to implement as the contiguous gold model but still accounts for the cytoplasm between GNPs. For the "peri" configuration, the gold-containing region is modelled as a spherical shell about the nucleus (with a $5 \mathrm{~nm}$ buffer between the shell and the nucleus or cytoplasm) with a thickness of 2.7-10.9 nm in the pure gold model and $50 \mathrm{~nm}$ 


\section{(a) peri}

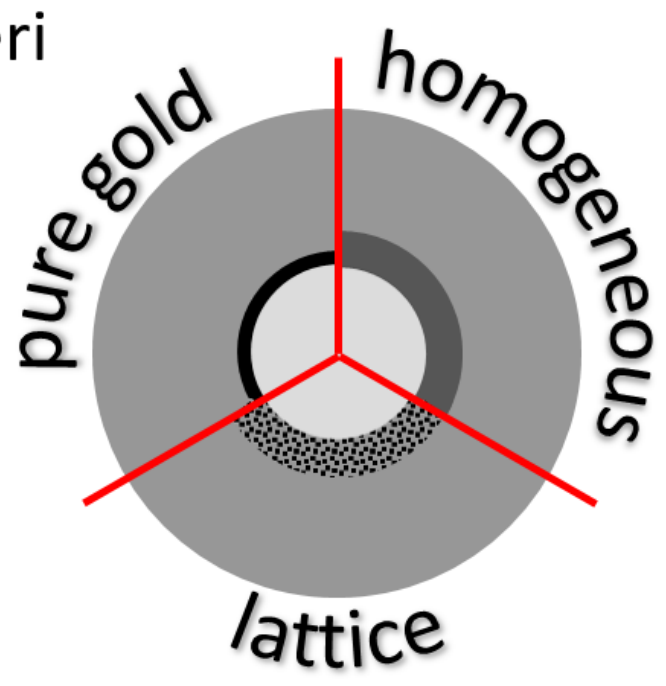

(b) endo

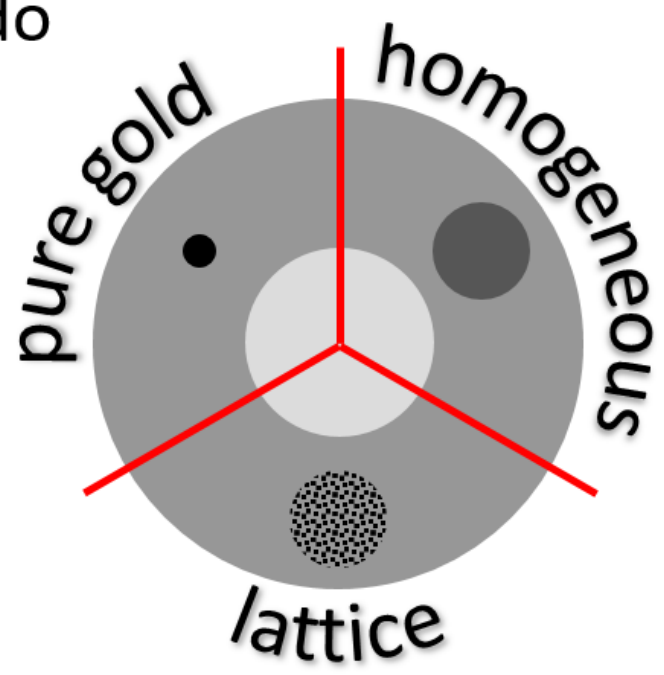

Figure 4.2: Diagram of three approaches to modelling gold in (a) a perinuclear shell or (b) an endosome; contiguous pure gold (top-left), homogeneous gold-tissue mixture (top-right) and a lattice of discrete GNPs (bottom) for each cell image. 
(diameter of a GNP) in the other two models, with GNP number density varying between $8.294 \times 10^{14}$ and $3.316 \times 10^{15} \mathrm{GNP} / \mathrm{cm}^{3}$. In either endosome case, the gold-containing region is modelled as a sphere with radius between 372 and $591 \mathrm{~nm}$ in the pure gold model and radius between 494 and $783 \mathrm{~nm}$ for the homogeneous and lattice model. GNP number density in the endosomes is always $6.457 \times 10^{15} \mathrm{GNP} / \mathrm{cm}^{3}$, the maximum density assuming $5 \mathrm{~nm}$ spacing between GNPs. All reference cell simulations are run for $10^{11}$ histories.

\subsection{2 $\mathrm{n}, \mathrm{cDEFs}$ for variable cell size}

Simulations are performed for the three gold configurations in section 4.2 .1 (i.e., "peri", "1endo", and "4-endo") using the discrete GNP modelling approach for different cell sizes. Cells with radii (and corresponding nucleus radii) of $5 \mu \mathrm{m}(2,3$ and $4 \mu \mathrm{m}), 7.35 \mu \mathrm{m}(4,5$ and $6 \mu \mathrm{m})$ and $10 \mu \mathrm{m}(7,8$ and $9 \mu \mathrm{m})$, chosen to represent a range of cell sizes, ${ }^{105}$ are investigated for concentrations of 5, 10 and $20 \mathrm{mg} / \mathrm{g}$. The 5 and $10 \mu \mathrm{m}$ radius cells are assumed to maintain the same nearest neighbour distance $(2.06 \mu \mathrm{m})$, thus the cell number density for the larger and smaller cells will be $8.06 \times 10^{8}$ and $1.32 \times 10^{8}$ cells $/ \mathrm{cm}^{3}$, respectively, ${ }^{105}$ as opposed to the $3 \times 10^{8}$ cells $/ \mathrm{cm}^{3}$ used for the reference cell. ${ }^{82}$ To ensure the total gold concentration throughout the medium remains the same, small and large cell GNP uptake is adjusted to compensate for the difference in volume occupied by the cytoplasm. For example, following equation (4.1), the number of $25 \mathrm{~nm}$ radius $\mathrm{GNPs}\left(n_{\mathrm{GNP}}\right)$ in a $5,7.35$ or $10 \mu \mathrm{m}$ radius cell would be 19,624, 52,723, and 120,741, respectively, to achieve a concentration of $20 \mathrm{mg} / \mathrm{g}$. Cell simulations are run for $10^{11}$ histories. 


\subsection{Results}

\subsubsection{Modelling approach to GNPs within a cell}

Figure 4.3 compares DEF to both the nucleus and cytoplasm of the reference cell as a function of energy when modelling gold using the methods described in section 4.2.1. Results are shown for the "peri" and "4-endo" configurations at $20 \mathrm{mg} / \mathrm{g}$ gold concentration. For the "peri" configuration, both nucleus and cytoplasm DEFs (n,cDEFs) are highest when gold is modelled contiguously; 3.5-10.7\% (5 mg/g) and 7.5-12.9\% (20 mg/g) higher than the analogous lattice model DEFs. The homogeneous model DEFs are less than pure gold but more than lattice model n,cDEFs; 2.4-6.7\% (5 mg/g) and 3.7-10.3\% (20 mg/g) higher than the lattice model DEFs. It is apparent that the three different modelling approaches yield different results for most DEFs above 2 for the "peri" configuration. This sensitivity to modelling approach is caused by the short distance between a point in the gold-containing region and the nearest scoring volume.

For the "4-endo" configuration, there is good agreement between the homogeneous mixture and lattice model nDEFs and cDEFs (within $<1 \%$ for most energies), with larger differences (ranging 1.2-5.5\%) at energies above the L- ( 13.3 keV) and K-edges (80.7 keV) of gold. n,cDEFs for the pure gold model (where the endosomes themselves are much smaller) are as much as $16 \%$ lower than the lattice DEFs. These discrepancies are due to the conservation of total gold mass in the cell between the different modelling approaches. The contiguous gold endosomes have $43 \%$ of the volume ( $54 \%$ of the surface area) of the two other models. Simulations of the pure gold case are also performed where the contiguous gold containing region occupies the same volume as the other two models (increasing the total mass of gold in the cell), for which the hexagonal lattice n,cDEFs were only 0.3-5.7\% higher than the pure gold model.

The n,cDEF as a function of energy trends seen in figure 4.3 also occur at concentrations 
of 5 and $10 \mathrm{mg} / \mathrm{g}$ but with lower absolute DEFs. The results for the "1-endo" configurations (for gold concentrations of 5 and $10 \mathrm{mg} / \mathrm{g}$ ) exhibit the same modelling differences as the "4-endo" configuration.

Though there is good agreement between different modelling approaches in many cases, the discrepancy between the three models is quite large for the cases for which DEF is highest. Therefore, moving forward in this work, only the hexagonal lattice of discretely modelled GNPs is used to model gold in the gold-containing regions, as it is the most realistic representation of GNPs in the cell.

\subsection{2 n,cDEF variation with GNP configuration and energy}

The discrete GNP model data in figure 4.3 is further expanded in figure 4.4, which shows nucleus and cytoplasm DEFs for a wider range of energies, configurations, and concentrations. The largest DEFs (for any concentration or configuration) are at 20 and $30 \mathrm{keV}$, energies right above the L-edge ${ }^{18}$ of gold. DEFs are also large at $90 \mathrm{keV}$ (above the K-edge ${ }^{18}$ ) but are markedly lower than at the L-edge energies. The DEFs for the "1-endo" configuration at $20 \mathrm{mg} / \mathrm{g}$ are omitted as the required endosome size is larger than what can fit in the reference cell cytoplasm. DEFs calculated with the "peri" configuration are either equal to or higher than either endosome configuration at all energies for the same concentration. Although by a small margin, the "4-endo" configuration DEFs are slightly larger than the "1-endo" DEFs. 

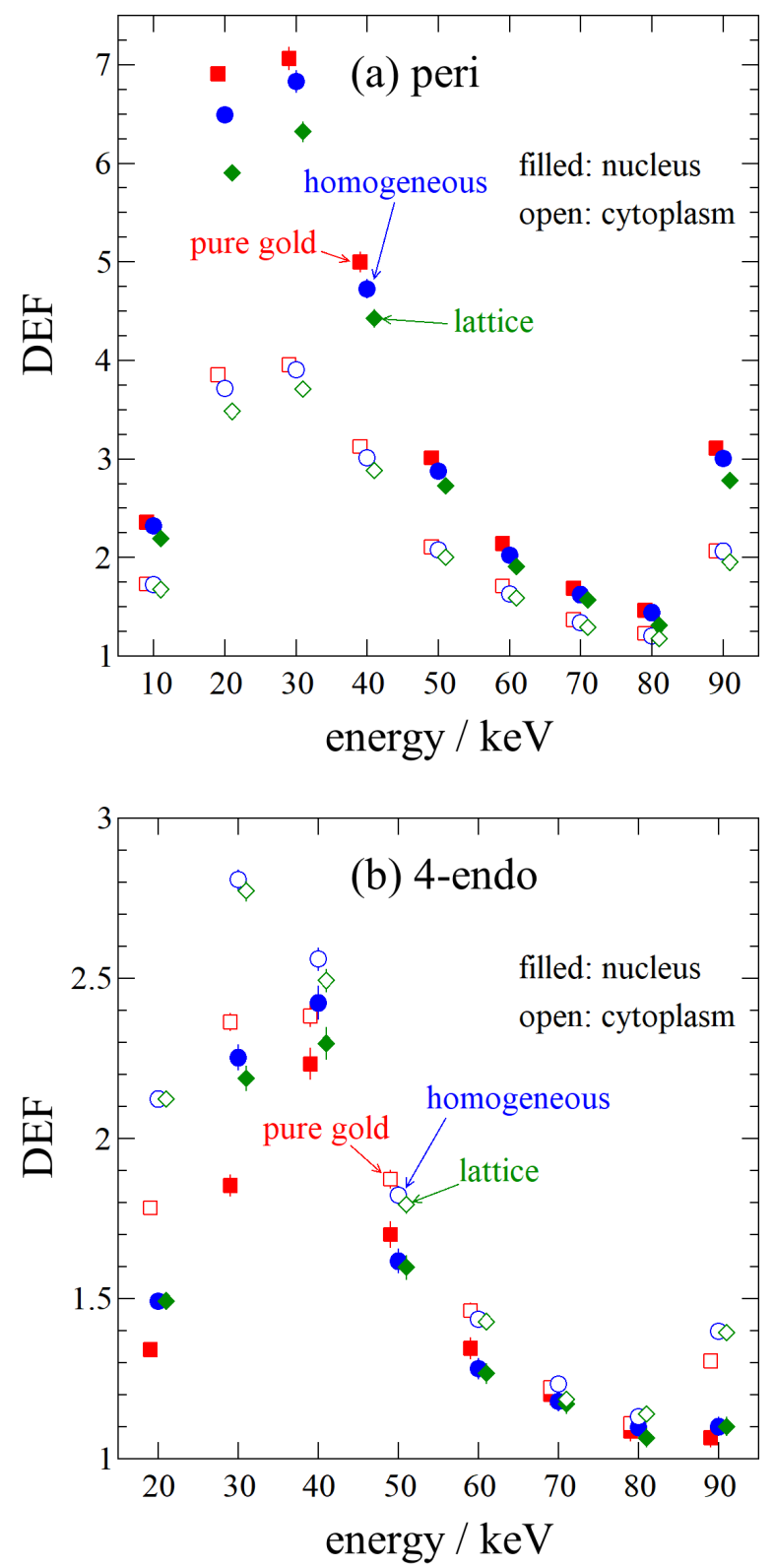

Figure 4.3: Nucleus and cytoplasm DEF of the $\left(r_{\text {cell }}, r_{\text {nuc }}\right)=(7.35,5) \mu \mathrm{m}$ reference cell for the (a) perinuclear and (b) four endosome gold configurations when gold (20 mg/g concentration) is modelled using contiguous gold (red squares - offset in $-x$ ), a homogeneous gold-tissue mixture (blue circles) or a hexagonal lattice (green diamonds - offset in $+x$ ). 

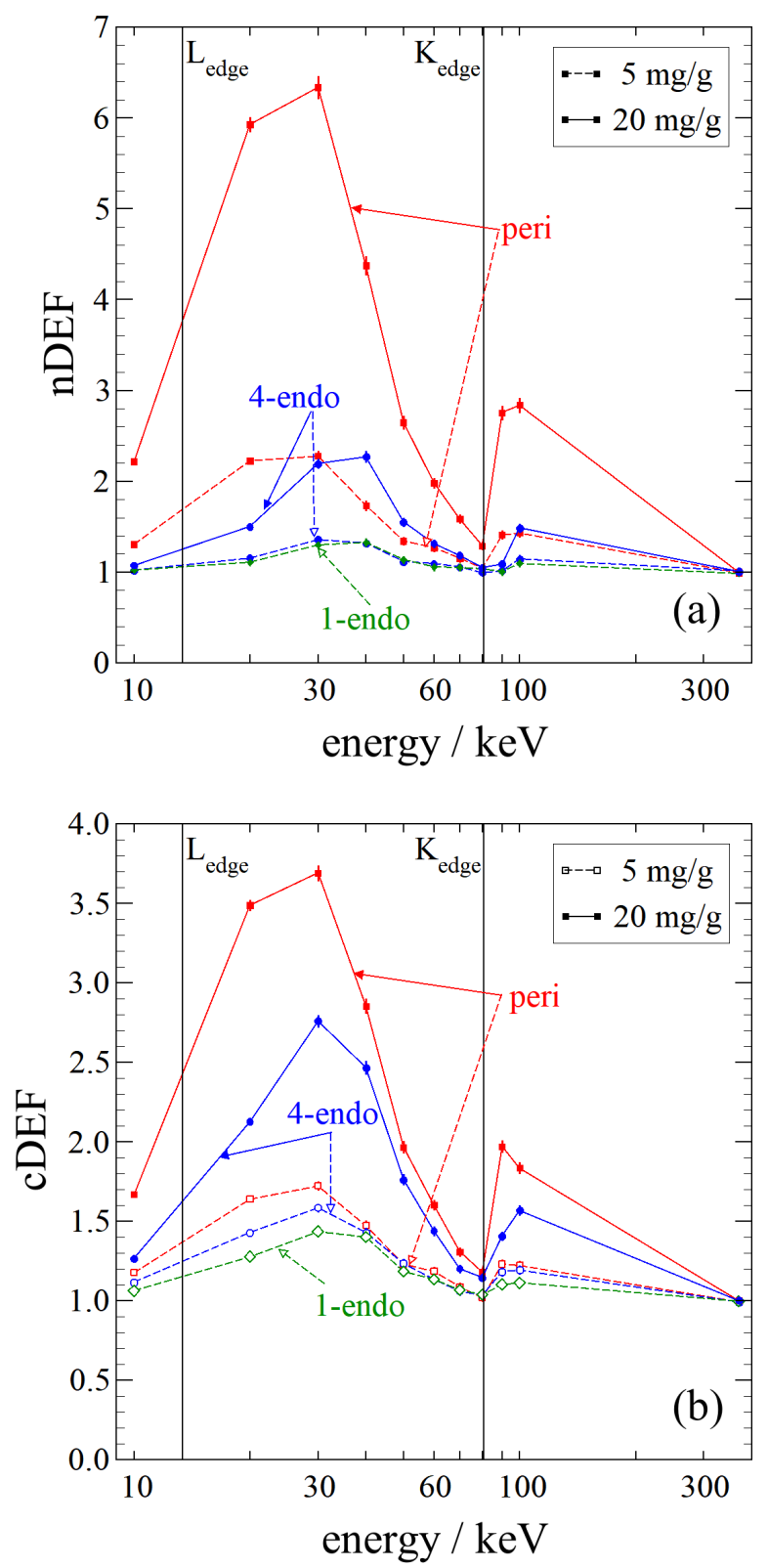

Figure 4.4: DEF for (a) nucleus and (b) cytoplasm for the $\left(r_{\text {cell }}, r_{\text {nuc }}\right)=(7.35,5) \mu \mathrm{m}$ reference cell as a function of energy for three gold configurations and concentrations of 5 and $20 \mathrm{mg} / \mathrm{g}$. 


\subsection{3 n,cDEF variation with GNP configuration and gold concen- tration}

Figure 4.5 plots nucleus and cytoplasm DEF of the reference cell as a function of gold concentration for the "peri", "4-endo", and "1-endo" configurations along with a linear fit of the data. As before, "peri" configuration DEF is consistently higher than the other configurations for all gold concentrations. Endosome configuration nDEFs for $90 \mathrm{keV}$ energies are close to unity for most concentrations, increasing to only $\sim 1.1$ for $24 \mathrm{mg} / \mathrm{g}$ concentrations.

The parameters of the linear least squares fit of the data in figure 4.5 are compiled in table 4.1. Most of the DEFs computed lie on the lines of best fit (within uncertainty); the (absolute) sum of DEF residuals range from 0.027 to 0.17 . Though the fits are good predictors for the investigated range of $4-24 \mathrm{mg} / \mathrm{g}$, several of the intercepts in the fit are significantly above or below one (DEF for $0 \mathrm{mg} / \mathrm{g}$ is one), most notably the endosome configurations at $20 \mathrm{keV}$ suggesting these fits are not reliable at very low concentrations.

Table 4.1: Linear fit parameters relating gold concentration to $\mathrm{n}, \mathrm{cDEF}$ of the $\left(r_{\text {cell }}, r_{\text {nuc }}\right)=(7.35$, 5) $\mu \mathrm{m}$ reference cell computed using the least squares method plotted in figure 4.5 , with slope in units of $\mathrm{g}_{\text {tissue }} / \mathrm{mg}_{\mathrm{Au}}$. Statistical uncertainty $(1 \sigma)$ on the final digit(s) for slopes is $\leq 0.001 \mathrm{~g} / \mathrm{mg}$, and for intercepts is indicated in parentheses.

\begin{tabular}{|c|c|c|c|c|c|c|c|}
\hline \multirow{2}{*}{$\begin{array}{r}\text { energy } \\
(\mathrm{keV})\end{array}$} & \multirow{2}{*}{ target } & \multicolumn{2}{|r|}{ "peri" } & \multicolumn{2}{|c|}{ "4-endo" } & \multicolumn{2}{|c|}{ "1-endo" } \\
\hline & & slope & intercept & slope & intercept & slope & intercept \\
\hline \multirow{2}{*}{20} & $\mathrm{nDEF}$ & 0.245 & $0.991(0.019)$ & 0.019 & $1.025(0.006)$ & 0.019 & $1.025(0.006)$ \\
\hline & $\mathrm{cDEF}$ & 0.116 & $1.115(0.019)$ & 0.041 & $1.290(0.021)$ & 0.026 & $1.152(0.013)$ \\
\hline \multirow{2}{*}{30} & $\mathrm{nDEF}$ & 0.093 & $0.862(0.022)$ & 0.034 & $0.993(0.015)$ & 0.034 & $0.993(0.015)$ \\
\hline & $\mathrm{cDEF}$ & 0.055 & $0.927(0.014)$ & 0.037 & $1.077(0.009)$ & 0.033 & $1.037(0.008)$ \\
\hline \multirow{2}{*}{50} & $\mathrm{nDEF}$ & 0.088 & $0.980(0.020)$ & 0.005 & $0.983(0.010)$ & 0.005 & $0.983(0.010)$ \\
\hline & $\mathrm{cDEF}$ & 0.047 & $0.987(0.007)$ & 0.015 & $1.099(0.007)$ & 0.010 & $1.050(0.008)$ \\
\hline
\end{tabular}



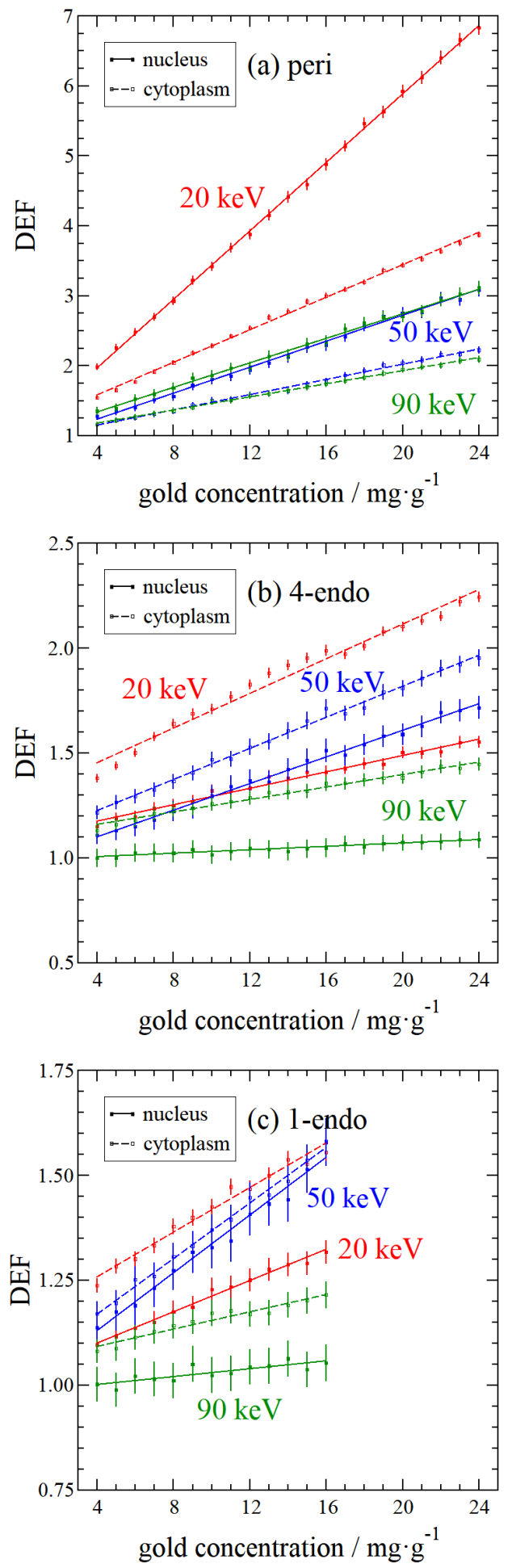

Figure 4.5: Nucleus and cytoplasm DEF of the $\left(r_{\text {cell }}, r_{\text {nuc }}\right)=(7.35,5) \mu \mathrm{m}$ reference cell as a function of concentration for 20,50 and $90 \mathrm{keV}$ monoenergetic photon sources for (a) "peri", (b) "4-endo", and (c) "1-endo" configurations with linear fit (table 4.1). "1-endo" results for concentrations of $17 \mathrm{mg} / \mathrm{g}$ or higher are omitted as the endosomes are too large to fit in the cytoplasm. 


\subsection{4 n,cDEF of cells with variable size}

Figure 4.6 shows the range of nucleus and cytoplasm DEFs for nine different cell sizes: $\left(r_{\text {cell }}\right.$, $\left.r_{\text {nuc }}\right)=(5,2),(5,3),(5,4),(7.35,4),(7.35,5),(7.35,6),(10,7),(10,8)$, and $(10,9) \mu m$. For both nucleus and cytoplasm, the highest DEFs correspond to the $(5,2) \mu \mathrm{m}$ cell in most cases and the lowest DEFs correspond to the $(10,7)$ um cell. For most "1-endo" (and some "4-endo") configurations, cells which could not fit the required size endosome within the cytoplasm are omitted. For the "1-endo" configuration at $20 \mathrm{mg} / \mathrm{g}$ concentration, only 3 cells make up the total data range and the reference cell is among the omitted.

Similar trends to those in figure 4.3 can be seen across all cell permutations, i.e., the quartile DEFs follow the same trend as the reference cell as a function of energy. The largest range of DEFs over cell/nucleus size occur at energies and with gold configurations for which the reference cell DEFs are highest, e.g., nDEF can range over as much as 2.99 to 21.5 in the "peri" configuration at $20 \mathrm{keV}$ for $20 \mathrm{mg} / \mathrm{g}$. The smallest ranges of DEFs occur about small reference cell DEFs, e.g., cDEF ranges over 1.20 to 1.38 in the "4-endo" configurations at $80 \mathrm{keV}$ for $20 \mathrm{mg} / \mathrm{g}$. At concentrations of $20 \mathrm{mg} / \mathrm{g}$, the "1-endo" DEF ranges are often even smaller than the equivalent "4-endo" ranges; this is primarily due to the exclusion of cell sizes where the endosome cannot fit, lowering the overall number of DEFs used to populate the range.

It is typically the upper most quartile that corresponds to the largest DEF range and the lower most quartile that corresponds to the smallest. Thus, the range of the inner quartiles (25-75\%) presents a much smaller range of DEFs overall, omitting the upper most quartile and its large range of DEFs (75-100\%), with a lower limit (25\%) still close to that of the nine cell statistical population $(0 \%)$. This range is a more clinically relevant variation one should expect in GNPT for tumour treatment planning (as overdosing by a factor of 2 or more is not typically a concern), but may be a dangerous underestiamte of DEF if used for non-tumour volumes such as organs-at-risk. The aforementioned 2.99 to 21.49 total 
$\mathrm{nDEF}$ variation is only 3.72 to 6.76 across the two inner quartiles (excluding the highest and lowest $25 \%$ of doses). The DEF of the reference cell (which has the median cell and nucleus size) in figure 4.3 is not the median DEF in most cases. Though it can fluctuate, the reference n,cDEFs are typically within the inner quartiles close to the median, with the most exceptions occurring in scenarios with cell sizes excluded from the (statistical) population because the endosome is too large to fit in the cytoplasm.

Figure 4.7 plots nucleus and cytoplasm DEF as a function of energy (analogous to figure 4.4) for the small (red, $5 \mu \mathrm{m}$ ) and large (blue, $10 \mu \mathrm{m}$ ) cells with all three of their corresponding nucleus sizes, along with the reference n,cDEF (green) for comparison. Data are shown for GNPs in a "peri" configuration with a concentration of $20 \mathrm{mg} / \mathrm{g}$, the scenario in which DEFs are highest. The figure shows that nDEFs (for all energies) increase as nucleus size decreases and cDEFs decreases as nucleus size decreases. The general trends (as a function of energy) exhibited are the same for all cell sizes, but at 20 and $90 \mathrm{keV}(10 \mathrm{keV}$ above the L- and K-edges, respectively) there is a much larger (relative) increase in DEFs for cells for which the scoring volume is smaller, i.e., high nDEFs when the nucleus is relatively small and high cDEFs when the nucleus is large (therefore cytoplasm volume is small). 

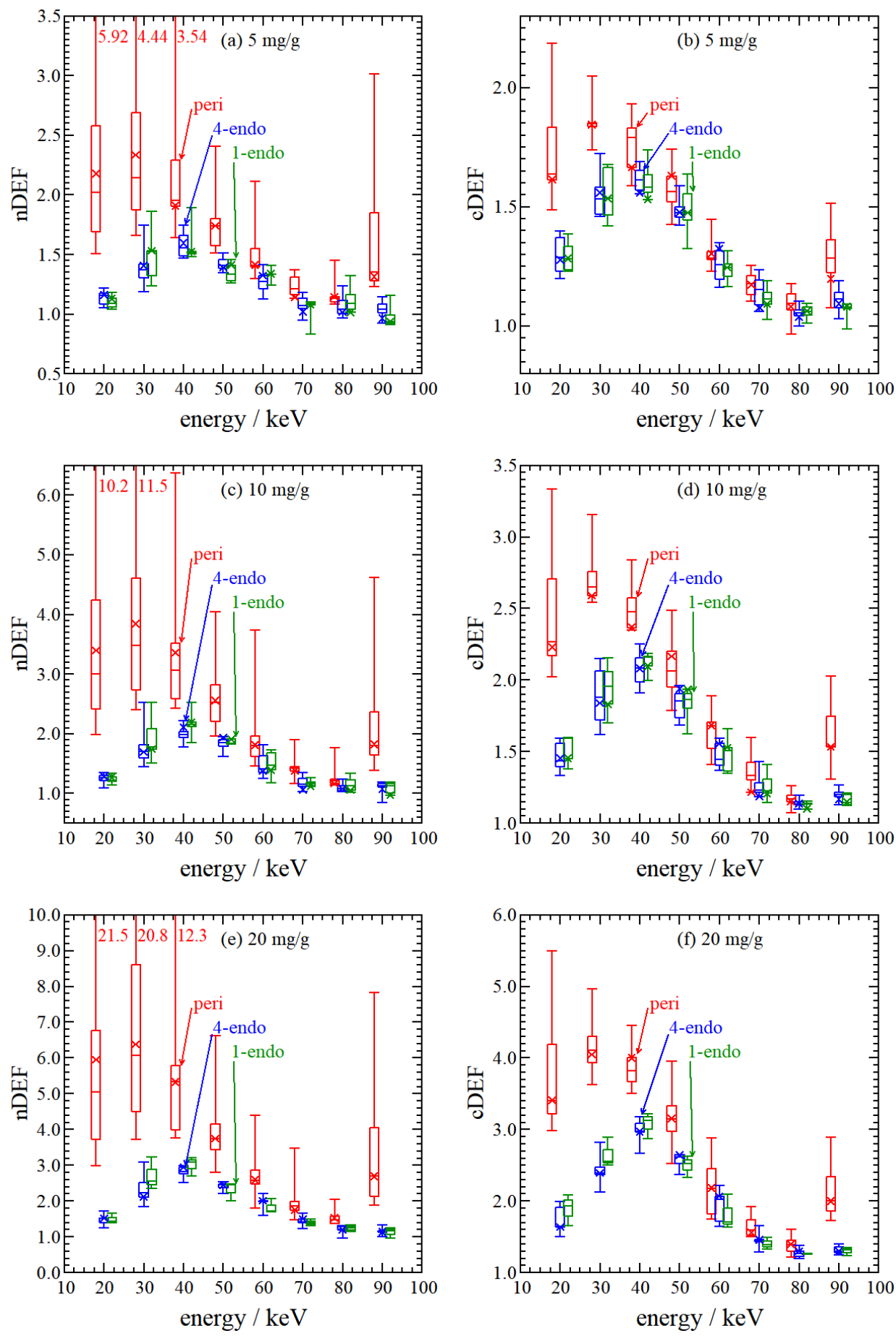

Figure 4.6: Box and whisker plots showing n,cDEF for (up to) 9 different cell/nucleus radii for gold configurations of $(a, b) 5 \mathrm{mg} / \mathrm{g},(\mathrm{c}, \mathrm{d}) 10 \mathrm{mg} / \mathrm{g}$, and $(\mathrm{e}, \mathrm{f}) 20 \mathrm{mg} / \mathrm{g}$. The whiskers represent the total range of DEFs amongst the 9 (or fewer) permutations, the boxes represent the inner quartiles $(25-75 \%)$, the horizontal line is the median and the ' $x$ ' is for the $\left(r_{\text {cell }}, r_{\text {nuc }}\right)=(7.35$, 5) $\mu \mathrm{m}$ reference cell. 

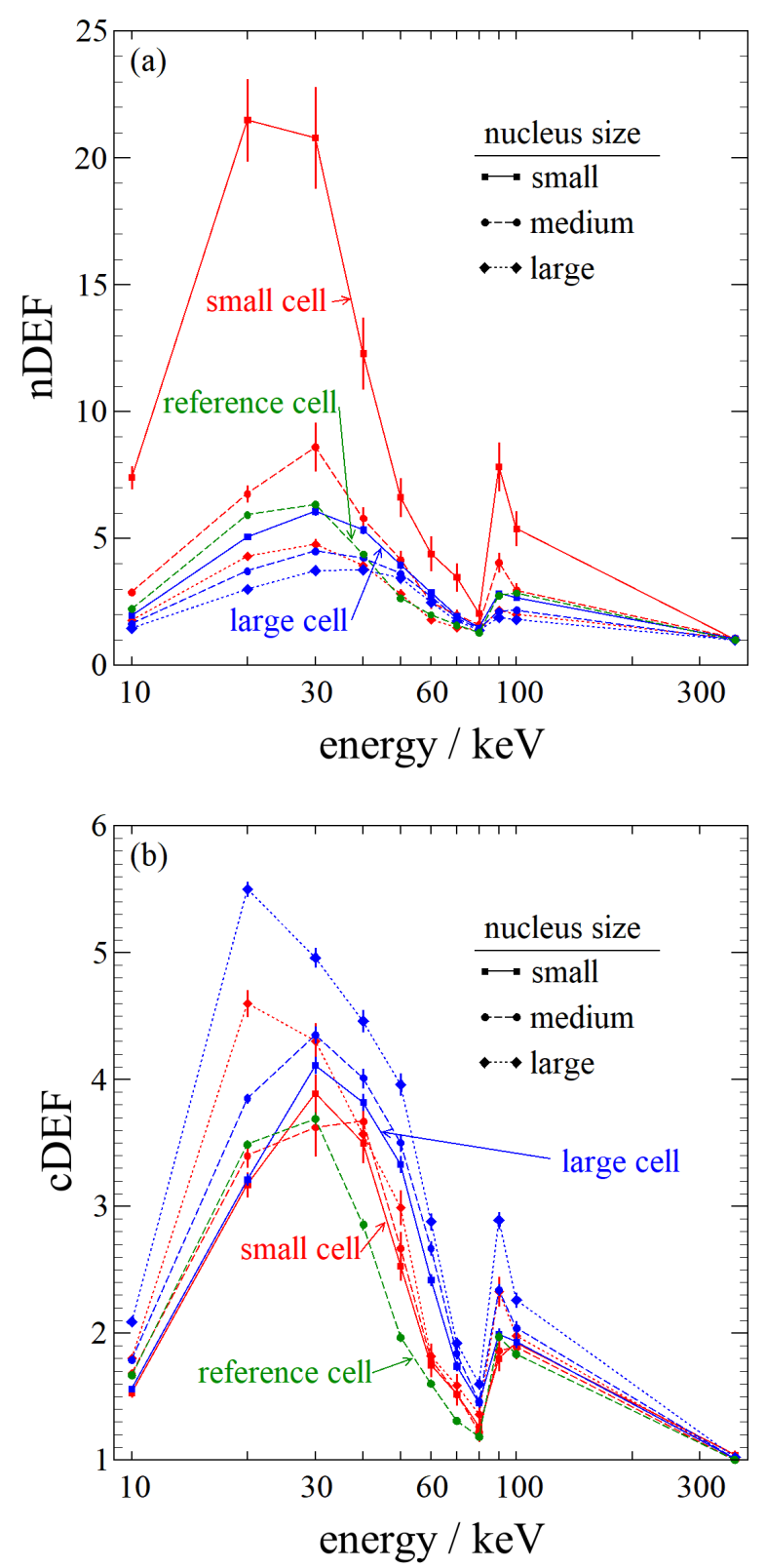

Figure 4.7: DEFs for (a) nucleus and (b) cytoplasm of the (red) small cells, $\left(r_{\text {cell }}, r_{\text {nuc }}\right)=(5$, $2),(5,3),(5,4)$, and the (blue) large cells, $(10,7),(10,8)$, and $(10,9) \mu \mathrm{m}$, as well as the (green) reference cell, $(7.35,5) \mu \mathrm{m}$, as a function of energy for $20 \mathrm{mg} / \mathrm{g}$ and gold in the "peri" configuration. 


\subsection{Discussion}

The research presented in this chapter uncovered a considerable range of cell nucleus and cytoplasm DEFs by investigating different gold configurations, gold concentrations, cell/nucleus sizes, and incident photon energies, as well as different MC approaches to modelling GNPs in cells. The hexagonal lattice of discrete GNPs model was thought to be to be the most appropriate choice for calculating n,cDEFs for all scenarios as it models GNPs discretely. GNPs localized in the perinuclear region lead to the largest DEF for both nucleus and cytoplasm. The increase in nDEFs and cDEFs as a function of gold concentration is linear for concentrations ranging 4 to $24 \mathrm{mg} / \mathrm{g}$, regardless of configuration or energy. The variation in $\mathrm{n}, \mathrm{cDEF}$ as a function of cell/nucleus size shows a significant dependence on scoring volume size. Though some of these scenarios have been investigated to some extent

in other MC studies of DEF on a cellular scale, ${ }^{58,60-62,106,107}$ many of them investigated a very limited parameter space and only use a single modelling approach to GNPs within a cell.

When comparing different gold configurations within a cell, "peri" n,cDEFs are larger than or equal to "1-endo" and " 4 -endo" n,cDEFs for the same gold concentration and energy. There are several effects at play in the "peri" model to cause this increase: the perinuclear region is in close proximity to both the nucleus and cytoplasm scoring volumes, the endosomes self-absorb many of the photoelectrons not generated in the periphery, ${ }^{111}$ and the "peri" configuration gold-containing region occupies a larger volume. Although there are some discrepancies when comparing cDEF between the "1-endo" and " 4 -endo" configurations, such as in table 4.1 and figure 4.6, for any one energy and concentration, the cDEF discrepancy is not much larger than the statistical uncertainty of either cDEF. Thus, because of the small discrepancies and the inability to fit a single endosome in a cell for a $20 \mathrm{mg} / \mathrm{g}$ concentration using our model, many of the "1-endo" results were omitted. 
Section 4.3.1 outlines many of the physical differences in modelling approaches investigated, such as the proximity of the gold-containing region to the scoring volume in the "peri" configuration. For the "peri" scenario there are many effects at play that differ between the modelling approaches. The contiguous and homogeneous models do not provide gaps of only tissue (photons entering the gold scoring-region are guaranteed to travel through some gold). Additionally, the three models affect photoelectrons generated in the gold differently. Photoelectrons generated in the contiguous and discrete model need to travel through some amount of gold (density of $19.32 \mathrm{~g} / \mathrm{cm}^{3}$ ), a $3-11 \mathrm{~nm}$ thick gold shell for contiguous and one (or more) $50 \mathrm{~nm}$ diameter thick GNPs; whereas, photoelectrons generated in the homogeneous model do not travel in gold, but rather in the mixture (density of 1.005-1.019 g/ $\mathrm{cm}^{3}$ ). This will impact the loss of energy of the photoelectrons before they potentially reach the scoring regions (or even the local GNP gold volume ${ }^{55}$ ), affecting the overall DEFs.

For the "1-endo" and "4-endo" configurations, the above effects are rather minimal as most photoelectrons that aren't generated in the periphery will be self-absorbed and much of the nanostructure details which were important in the $50 \mathrm{~nm}$ thick (or less) perinuclear shell average out over the (relatively) large micron-scale endosomes. When comparing the speed of the simulations, the contiguous and homogeneous cell models ran at effectively the same efficiency. The discrete model introduced a drop of 11.4-23.9\% in efficiency with transport occurring exclusively within the cell (no extracellular regions), which, in simulations with extracellular volume, would be a very small overall decrease in efficiency.

Though the contiguous and homogeneous gold models do agree with the discrete GNP model DEFs in many scenarios, the discrete model utilizes the most realistic representation of GNPs in cells across all parameters at only a small efficiency cost and is recommended for general use in MC cell modelling. Simulations were also performed by placing whole GNPs (as opposed to the often partial GNPs modelled in the lattice configuration) in a spiral grid ${ }^{112}$ in the "peri" configuration using the default EGSnrc geometries. The spiral 
grid model ran at $99.995 \%$ slower than the contiguous gold model, and introduced errors in the simulations at concentrations as low as $5 \mathrm{mg} / \mathrm{g}$ due to the large memory requirements for individually modelled GNPs and difficulty placing GNPs at the cell's poles. For the few successful simulations at $2 \mathrm{mg} / \mathrm{g}$ or less, DEFs did agree with the discrete model for every energy investigated.

As shown in figure 4.5, the relationship between an individual (i.e., a single cell with little surrounding scatter medium) cell DEF and gold concentration is near linear for the range of concentrations explored in this work $(4-24 \mathrm{mg} / \mathrm{g})$. Therefore, in a local model of a single cell it is possible to calculate DEF as a factor of gold concentrations for different cell sizes, gold configurations and energies. This is mostly due to the cell model constraining the gold-containing region to be the same for the "peri" configuration and to be in a fixed location in the endosome configurations. Unfortunately, expanding the scenario to a tumoursized volume, the difference in attenuating effects throughout the scatter media must also be taken into account, as these affect local DEF (as seen in chapter 3). Select concentrations of $1 \mathrm{mg} / \mathrm{g}$ and less were investigated and were found to not follow the same linear trend for the concentrations of $4 \mathrm{mg} / \mathrm{g}$ and above, which is why several of the lines defined in table 4.1 do not have an intercept of 1 . The results are omitted as computing enough DEFs to demonstrate trends at concentrations below $1 \mathrm{mg} / \mathrm{g}$ proved computationally prohibitive due the small uncertainties needed to quantify the very small dose enhancement.

Often, comparison to other works in the literature are difficult due to the multitude of modelling choices required for modelling the source, phantom, cell, and GNPs; here we compare to the work of Douglass et $a l^{58}$ by extrapolating nDEF of a cell with matching nucleus size. Douglass et al calculated an nDEF of 55.7 (defined as Dose Enhancement Ratio in their paper) to a cell with GNPs in a "peri" configuration when irradiated by a $80 \mathrm{kVp}\left(40.6 \mathrm{keV}\right.$ average energy $\left.{ }^{113}\right)$ beam. Their cell model consisted of an ellipsoidal cell boundary (randomly sampled 9-13 $\mu \mathrm{m}$ semimajor axis length) with a small nucleus (3 $\mu \mathrm{m}$ 
radius) embedded within it surrounded by a $300 \mathrm{~nm}$ thick perinuclear shell of contiguous gold. This quantity of gold would be 10.85 times larger than the highest considered in this work (assuming reference cell density of $3 \times 10^{8}$ cells $/ \mathrm{cm}^{3}$ ), averaging out to a tumour gold concentration of $216 \mathrm{mg} / \mathrm{g}$ (magnitudes larger than some of the highest proposed concentrations of $30,{ }^{63} 43,{ }^{67}$ and $66 \mathrm{mg} / \mathrm{g}^{26}$ in the GNPT literature). Using the linear relationship demonstrated in table 4.1 , the extrapolation of $\left(r_{\text {cell }}, r_{\text {nuc }}\right)=(5,3) \mu \mathrm{m}$ cell nDEFs at 5 and $20 \mathrm{mg} / \mathrm{g}$ concentrations predicts an nDEF of 57.9 at $216 \mathrm{mg} / \mathrm{g}$, only $4.0 \%$ higher than that calculated by Douglass et al.

In figure 4.7, the DEF trends over energy for various cell sizes are similar to those of the reference cell. There is a large change in DEF with cell radii, which is caused by the GNP uptake for different cell concentration with cell radius (see equation (4.1)). For cells of the same radius but with different nucleus radii, there is an additional effect caused by the relative proximity of the scoring volumes to the gold-containing regions (i.e., scoring volume in the small nuclei is, on average, closer to the GNPs in the "peri" configuration). Outside of the aforementioned effects, there is also a difference in the n,cDEF trends with energy at points above the L- (20 and $30 \mathrm{keV}$ ) and K-edges (90 and $100 \mathrm{keV}$ ). For example, cDEFs of the $(10,7) \mathrm{\mu m}$ cell when going from 20 to $30 \mathrm{keV}$ are 5.5 to 5.0 (0.5 decrease in DEF) and for the $(10,9) \mu \mathrm{m}$ cDEFs are 3.2 to 4.1 (0.9 increase in DEF).

This discrepancy in cDEF trends going from $20 \mathrm{keV}$ to $30 \mathrm{keV}$ for different nucleus radii for the large cell stems from several competing effects at this energy range. The first has to do with the photelectric cross section of gold, which decreases with energy above the L-edge $(\sim 13.3 \mathrm{keV})$, leading to fewer photoelectrons being generated at $30 \mathrm{keV}$ than $20 \mathrm{keV}$. The photoelectrons generated in the two cases will also have different ranges and stopping powers (10 and $20 \mathrm{keV}$ energy electrons have ranges of 2.5 and $8.6 \mu \mathrm{m}$ in water, respectively ${ }^{90}$ ). Often, the decrease in DEF due to the lower total number of photoelectrons at $30 \mathrm{keV}$ is outweighed by the increased energy deposited by the more energetic photoelectrons that are 
generated, leading to an overall increase in $\operatorname{DEF}($ e.g., $(10,7) \mu \mathrm{m}$ cell). This increase only occurs when the scoring volume is large enough that the more energetic (and long range) electrons still deposit most of their energy before leaving the volume. In cases where the scoring volume is very small (e.g., $(10,9) \mu \mathrm{m}$ cell with its $1 \mu \mathrm{m}$ thick cytoplasm shell), the more energetic electrons escape before depositing most of their energy. Thus, the increased energy of the photoelectrons generated at $30 \mathrm{keV}$ does not compensate for the lower number of total photoelectrons generated in the cell, leading to a drop in DEF going from 20 to $30 \mathrm{keV}$. This difference in $\mathrm{n}, \mathrm{cDEFs}$ trends is notable for several different cell/nucleus sizes when going from 20 to $30 \mathrm{keV}$ and 90 to $100 \mathrm{keV}$.

The single cell nDEF trends in the "peri" configuration (figure 4.4) are similar to those found by Rasouli and Masoudi ${ }^{106}$ in their heterogeneous gold-tissue mixture model and by Cai $e t a l^{61}$ in their concentric sphere cell model, but with differences in absolute value due to different cell parameters, gold concentrations and incident beam configurations.

All DEFs presented in this chapter have been calculated using the average doses to one of nine cell models. Though the range of DEFs with changing cell size can be used as a first order estimate of variation over a cell population, in typical microdosimetry studies, specific energy distributions are actually measured over a large population of cells. ${ }^{114}$ In other words, this chapter has focused on the expectation value of that distribution, presenting 768 expected values of the 4261 single cell DEF calculations performed. An investigation calculating the DEF in different scenarios as a probability distribution (analogous to specific energy distributions in microdosimetry) would be a natural progression of the work in this chapter. 


\subsection{Conclusion}

Cellular DEFs vary greatly with cell size, gold concentration, and incident source energy. The n,cDEFs were calculated using the lattice model as the discrete GNPs represent the most realistic model, though the contiguous volume of pure gold or homogeneous gold-tissue mixture model are adequate in some scenarios. DEFs can range from unity to as high as 21.5 and 5.5 for nDEF and $\mathrm{cDEF}$, respectively. Thus, when proposing novel GNPT treatments, many factors beyond the typical radiotherapy considerations are required, such as target cell size and GNP configuration in the cell of the proposed delivery method. Comparing perinuclear and endosome gold configurations, the large surface area and proximity to scoring volumes of GNPs in the perinuclear configuration lead to the largest DEFs for both the cytoplasm and nucleus at all energies and concentrations. nDEFs and cDEFs vary with cell size, and incident beam energy to maximize DEFs varies with cell size as well. The wide parameter space (all permutations of cell size, gold concentration, photon energy and gold configuration) investigated in this chapter is much broader than that explored in MC GNPT cell dosimetry studies, and the wide array of trends demonstrated herein can help to broaden the understanding of GNPT. 


\section{Chapter 5}

\section{Cell DEF in GNPT with HetMS}

\subsection{Introduction}

Studies that propose new GNPT treatments often avoid modelling both whole tumour volumes and microscopic scoring volumes due to the complexity of the required simulation geometry and the need to extract dose at many points. Brivio et $a l^{23}$ performed calculations for GNPs added in prostate brachytherapy using a homogeneous gold-tissue mixture to represent GNPs within the prostate. Asadi $e t a l^{63}$ distributed GNPs within an eye model for their proposed GNPT eye plaque brachytherapy treatment, and measured DEFs of bulk tissue (as opposed to cell models). The shortcomings of these models have been pointed out before, ${ }^{16,39,52}$ but even studies modelling targets on a cellular scale ${ }^{61,62}$ in larger scatter volumes are often limited to a fixed depth/position and with very few changes to their microscopic models. Thus, these simulations do not account for variations in DEF with distance from the source and the fluctuations in cell size ${ }^{115}$ and local GNP concentration. ${ }^{116}$

This chapter uses the HetMS model introduced in chapter 3 combined with the cell models studied in chapter 4 to efficiently compute nucleus and cytoplasm DEFs on tumour length scales. DEFs are computed for several gold concentrations, source energies, and GNP 
configurations in a large cylinder with a constant macroscopic gold concentration throughout it and a sphere with GNPs diffusing over time from its center. To quantify the aforementioned cell size and gold concentration fluctuation, calculations are performed to compute DEFs when introducing a fluctuation in gold concentration to a target cell and/or all of its neighbouring cells. The expected variation in DEFs with fluctuation in gold concentrations is then combined with the variations in DEF when fluctuating cell size (calculated in the previous chapter) to compute the expected range of DEFs for different GNPT scenarios. This expected range is then applied to the HetMS cylinder simulations to demonstrate the large variation in DEFs one would expect over a population of cells in GNPT.

\subsection{Methods}

The Heterogeneous MultiScale (HetMS) model, introduced in chapter 3, is used in this chapter to simulate GNPT scenarios. HetMS combines an efficient (in terms of particle transport) model for bulk media with detailed models for feature extraction in regions of interest into a single phantom. This chapter takes the cell models investigated in chapter 4 , and uses them to populate microscopic scoring regions which are then placed into a cylinder containing a constant macroscopic gold concentration or a sphere containing gold concentrations that decrease as a function of radius, two phantoms used in the introductory HetMS chapter with far simpler microscopic scoring regions.

All cells simulated in this chapter are modelled as a concentric nuclear sphere of radius $5 \mu \mathrm{m}$ within a cytoplasm sphere of radius $7.35 \mu \mathrm{m}$, referred to as the reference cell. The only media used in this chapter are ICRU four-component tissue (10.1\% hydrogen, $11.1 \%$ carbon, $2.6 \%$ nitrogen, and $76.2 \%$ oxygen by mass $^{20}$ ) for all non-gold biological media, pure gold used for GNPs or a homogeneous mixture of the two materials used for the bulk scatter media. A hexagonal close-packed lattice is used for modelling GNPs clustered within cells, 
to place cells within the microscopic scoring regions, and to model a 13 cell cluster, i.e., a target cell and its 12 neighbours. Cells are modelled with $2.06 \mu \mathrm{m}$ spacing between them and all 12 neighbouring cells, ${ }^{82,117}$ shown in figure 5.1, which equates to a reference cell number density of $3 \times 10^{8}$. Three different configurations are used to model GNPs for a cell containing gold; GNPs clustered closely about the nucleus ("peri") or the GNPs clustered tightly into a single ("1-endo") or four ("4-endo") compartment(s). A detailed description and diagram of these configurations is given in chapter 4 (section 4.2.1 and figure 4.1). EGSnrc transport parameters are the same as those used in chapter 2.

\subsubsection{Expected variation in cell DEF in GNPT}

A set of simulations are performed to quantify the DEF variations with a $20 \%$ fluctuation in target cell and/or all neighbouring cell gold concentration, computing a population of nine DEFs; this is analogous to the study of cell/nucleus size fluctuations in section 4.2.2 of chapter 4 . The $20 \%$ fluctuation is an arbitrary (ideal) fluctuation that is equivalent to the $20 \%$ fluctuation of nucleus radius for the reference cell in chapter 4 . For example, for a $5 \mathrm{mg} / \mathrm{g}$ concentration the nine permutations of combining target and neighbour concentrations $\left(c_{\text {target }}, c_{\text {neighbour }}\right)$ investigated are $(4,4),(4,5),(4,6),(5,4),(5,5),(5,6),(6,4),(6,5)$, and $(6,6) \mathrm{mg} / \mathrm{g}$. Simulations of a target cell and its 12 neighbouring cells, configured in a hexagonal close-packed lattice model with a nearest neighbour distance of $2.06 \mu \mathrm{m}$ between outer radii, ${ }^{82}$ are performed. The group of 13 cells is placed at the centre of a $100 \mu \mathrm{m}$ radius sphere containing only tissue. Simulations are performed for three gold configurations ("peri", "1-endo", and "4-endo"), concentrations of 5, 10, and $20 \mathrm{mg} / \mathrm{g}$, and 10-370 keV energies. Simulations are run for $4 \times 10^{10}$ histories and no variance reduction techniques are used.

The variation in cell (and nucleus) size and local gold concentration (to a target cell and/or its neighbours) is quantified in terms of an upward variation, $\% \Delta_{\mathrm{DEF}}^{+}$, and downward 
variation, $\% \Delta_{\mathrm{DEF}}^{-}$, defined as

$$
\begin{aligned}
& \% \Delta_{\mathrm{DEF}}^{+}=\frac{Q_{3}^{\mathrm{DEF}}-Q_{2}^{\mathrm{DEF}}}{\mathrm{DEF}_{\text {ref }}} \times 100 \% \\
& \% \Delta_{\mathrm{DEF}}^{-}=\frac{Q_{2}^{\mathrm{DEF}}-Q_{1}^{\mathrm{DEF}}}{\mathrm{DEF}_{\text {ref }}} \times 100 \%
\end{aligned}
$$

where $Q_{i}^{\mathrm{DEF}}$ is the DEF of the $i$ th quartile and $\mathrm{DEF}_{\text {ref }}$ is the DEF of the reference cell with a local gold concentration matching the overall gold concentration. Values are normalized as a percentage of the reference cell DEF so that they may later be used to determine an expected variation range of DEF using a calculation of nominal reference cell DEF. The inner quartiles are chosen as they significantly reduce the overall variation while still providing a close estimate of the lower bound, discussed further in section 4.3 .4 of chapter 4.

The second set of $\% \Delta_{\mathrm{DEF}}^{+}$and $\% \Delta_{\mathrm{DEF}}^{-}$are calculated using the statistical data from the DEFs calculated for various permutations of cell and nucleus size in section 4.3.4 (chapter 4). The quartile data are taken from the set of cells with sizes $\left(r_{\text {cell }}, r_{\text {nuc }}\right)=(5,2),(5,3)$, $(5,4),(10,7),(10,8)$, and $(10,9) \mu \mathrm{m}$, shown in figure 4.6. Data are taken for three gold configurations ("peri", "1-endo", and "4-endo"), concentrations of 5, 10, and $20 \mathrm{mg} / \mathrm{g}$, and $10-370 \mathrm{keV}$ energies. The separate $\% \Delta_{\mathrm{DEF}}^{+}$and $\% \Delta_{\mathrm{DEF}}^{-}$for both the cell/nucleus size and target/neighbour concentrations are then combined by quadrature sum. The resulting combined $\% \Delta_{\mathrm{DEF}}^{+}$and $\% \Delta_{\mathrm{DEF}}^{-}$(referred to together as $\% \Delta_{\mathrm{DEF}}$, the total expected variation) are then compiled, to be used to compute a range of expected DEFs over a cell population based on a nominal cell DEF calculation.

The variations for nucleus/cell size and target/neighbour gold concentration fluctuations are combined using quadrature addition (which assumes independence) even though both fluctuations can affect the GNP uptake of the cell ( $\left.n_{\mathrm{GNP}}\right)$. Equation (4.1) shows the relationship between $n_{\mathrm{GNP}}, N_{\text {cell }}$ (number of cells per gram of tissue) and $c$ (gold concentration); the two fluctuations (nucleus/cell and target/neighbour gold) are performed on the 
variables $N_{\text {cell }}$ and $c$, respectively. Thus the effect either fluctuation has on cell GNP uptake $\left(n_{\mathrm{GNP}}\right)$ is independent in our model.

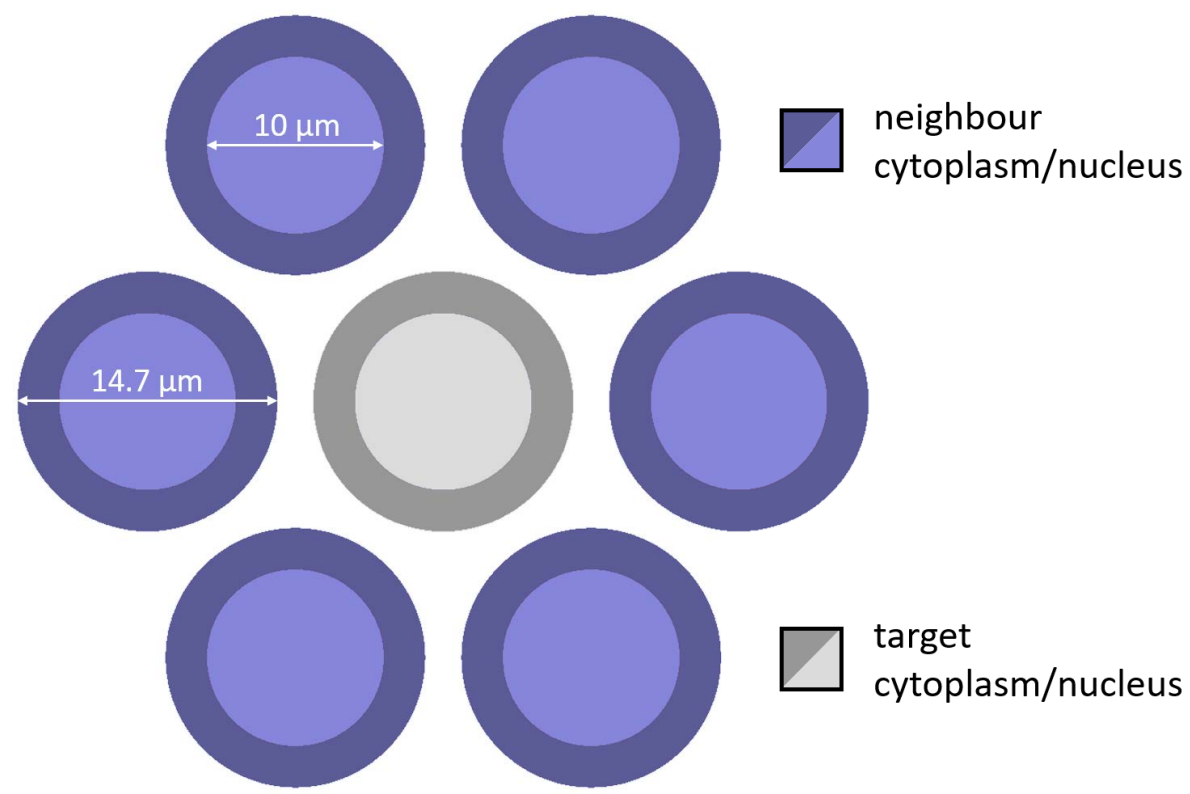

Figure 5.1: Cross-sectional diagram of a central scoring cell (grey) surrounded by all of its neighbouring cells (blue) in a close-packed hexagonal lattice configuration.

\subsubsection{Microscopic scoring regions containing cells for HetMS}

The microscopic scoring region used for HetMS simulations contains cell models; its average elemental composition is that of the single medium mixture of gold and tissue used in the bulk of the phantom. Cells are placed in a hexagonal close-packed lattice with $2.06 \mu \mathrm{m}$ spacing between outer radii (based on a number cell density of $3 \times 10^{8} \mathrm{cell} / \mathrm{cm}^{3}$ ). ${ }^{82}$ The total mass of gold in each cell is chosen such that the average mass of gold over mass of tissue in the scoring region matches the gold concentration (i.e., 5, 10, or $20 \mathrm{mg} / \mathrm{g}$ ) of the bulk tissue (see equation (4.1)). A buffer region spanning a cell diameter (14.7 $\mu \mathrm{m})$, where cells are still modelled but no nucleus or cytoplasm is scored in, is placed along every boundary that interfaces with macroscopic homogeneous media. The boundary ensures that only fully modelled cells are scored in, achieving proper local scatter conditions. Figure 5.2 shows a 
spherical microscopic scoring region cross section with a buffer region embedded in a goldtissue mixture, and figure 5.3 shows an equivalent scoring region for a spherical shell.

An additional microscopic scoring region is also used, similar to that in chapter 3, where GNPs are placed evenly (hexagonal lattice) throughout the same volume as the cellular microscopic scoring cavity to score dose to all the non-buffer tissue in the phantom, and will be referred to from here on as the dispersed scenario. When either scoring region is used in a simulation with a parallel beam as a source, the lattice is rotated such that the primary source direction does not align with any axis of symmetry of the lattice (avoiding shadowing effects).

\subsubsection{Tumour with constant macroscopic gold concentration}

A HetMS simulation is performed with a $3 \mathrm{~cm}$ long, $1 \mathrm{~cm}$ radius cylinder composed of a gold-tissue mixture (concentrations of 5, 10 or $20 \mathrm{mg} / \mathrm{g}$ ) irradiated by a parallel photon beam (monoenergies of $10-370 \mathrm{keV}$ as well as ${ }^{125} \mathrm{I},{ }^{131} \mathrm{Cs}$, and ${ }^{103} \mathrm{Pd}$ spectra ${ }^{98,100}$ ) incident over one of its flat faces. Microscopic scoring regions defined as $100 \mu \mathrm{m}$ radius spheres (including the $14.7 \mu \mathrm{m}$ buffer) are placed at 119 evenly spaced positions along the central cylindrical axis. A set of simulations are performed for which the microscopic scoring cavity is filled with dispersed $25 \mathrm{~nm}$ diameter GNPs in a hexagonal lattice, scoring dose to all tissue (analogous to the cubic lattice GNP simulations in chapter 3). Another set of simulations are performed with the scoring cavity defined in section 5.2.2 with cell models for each gold configuration ("peri", "1-endo" and "4-endo"), see figure 5.2. A set of simulations with only tissue are performed for DEF calculations using two microscopic models: cells containing no GNPS or a cavity filled with only tissue. Russian roulette with a factor of 64 (1 in 64 chance of survival and survivors have 64 times higher weight) is applied to all electrons that are at a distance from any scoring volume larger than their range under the continuous slowing down approximation. Simulations are run for $10^{10}$ histories. 


\section{cytoplasm GNP}

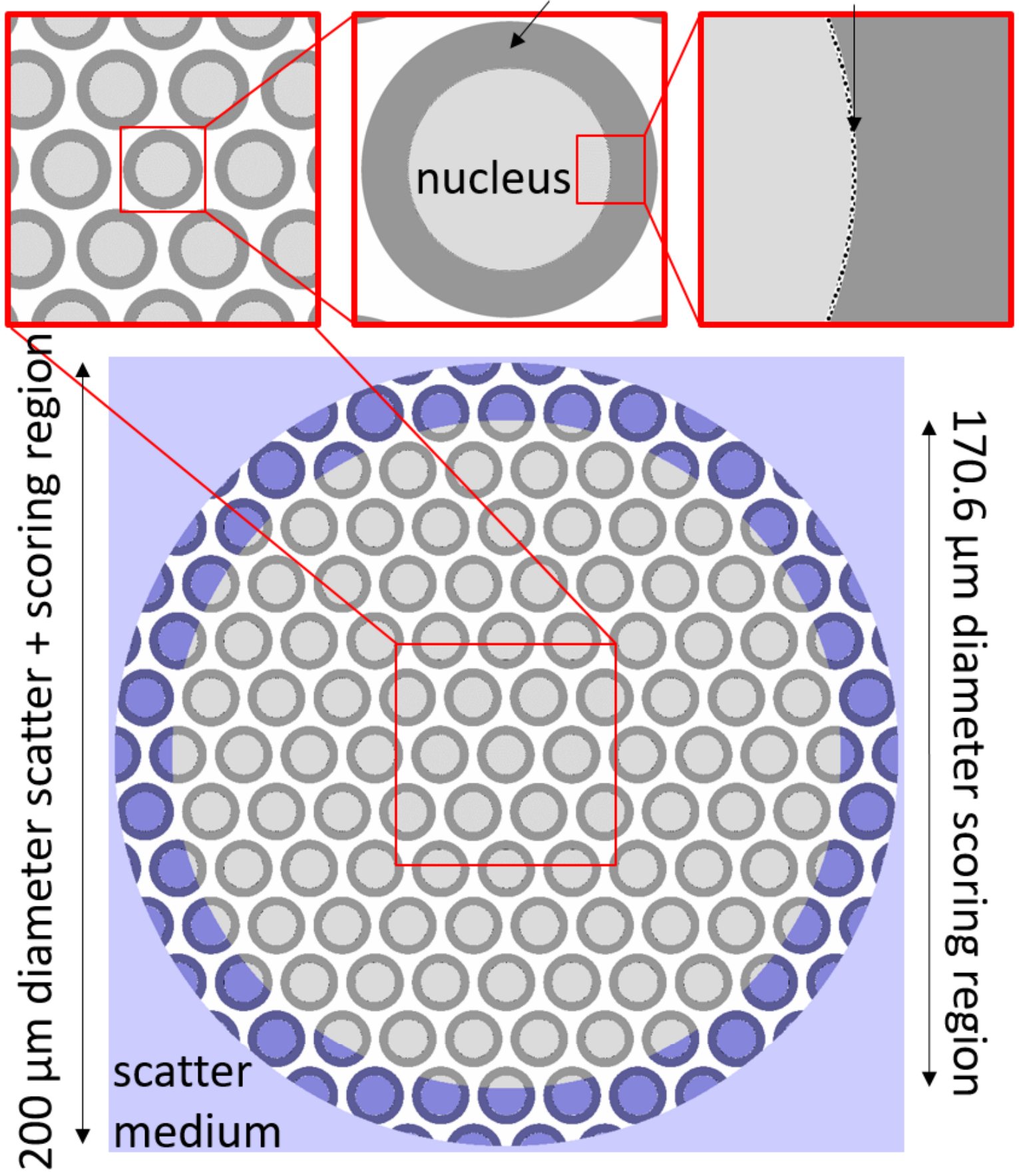

Figure 5.2: Microscopic scoring region (100 $\mu \mathrm{m}$ radius sphere) containing cells with GNPs in the perinuclear configuration generated using EGSnrc. Grey nucleus and cytoplasm are the scoring volumes, blue regions are the buffer. The sphere is embedded in a single, homogeneous scatter medium with matching average elemental composition. 


\subsubsection{Tumour with varying gold concentration}

A HetMS simulation is performed with a $2.5 \mathrm{~cm}$ radius sphere containing a GNP eluter and

point source in the centre. This model is based on the work of Sinha $e^{2} a l^{33}$ (a continuation of the work in chapter 3) where the sphere is divided into 51 subregions (a $0.025 \mathrm{~mm}$ thick region, 49 regions with a thickness of $0.05 \mathrm{~mm}$ and another $0.025 \mathrm{~mm}$ thick region), each with a different gold concentration based on the time the eluted GNPs took to diffuse to different radii. A plot of gold concentration as a function of radius at different times is shown in figure 3.2 of chapter 3 , described in more detail in section 3.2.3. A microscopic scoring region (100 $\mathrm{\mu m}$ thick shell) is placed at the centre of the $0.05 \mathrm{~mm}$ thick regions, which consists of cells with one of three configurations ("peri", "1-endo" and "4-endo") or dispersed GNPs in a hexagonal lattice. EGSnrc generated microscopic scoring cavity images are shown in figure 5.3. A simulation with only tissue (for DEF calculation) is performed with two different microscopic models: cells containing no GNPs or a cavity filled with only tissue. Typical brachytherapy spectra ${ }^{98,100}$ of ${ }^{125} \mathrm{I},{ }^{131} \mathrm{Cs}$, and ${ }^{103} \mathrm{Pd}$ and monoenergies ranging $10-370 \mathrm{keV}$ are used in an isotropic point source in the centre of the phantom. Simulations are run for $10^{10}$ histories and no variance reduction techniques are used.

\subsection{Results}

\subsubsection{Expected variation in cell DEF in GNPT}

In simulations of the 13 cell cluster, the differences in DEFs when varying the concentration of the 12 neighbouring cells by $20 \%$ is found to be very small (0-2\% differences in DEFs) compared to DEFs when varying target cell concentration (5-21\% differences in DEFs). The differences when varying the target cell gold by $20 \%$ agrees with the results found in figure 4.5 of chapter 4, where DEFs varied near linearly with gold concentration. 


\section{$70.6 \mu \mathrm{m}$ thick scoring region}

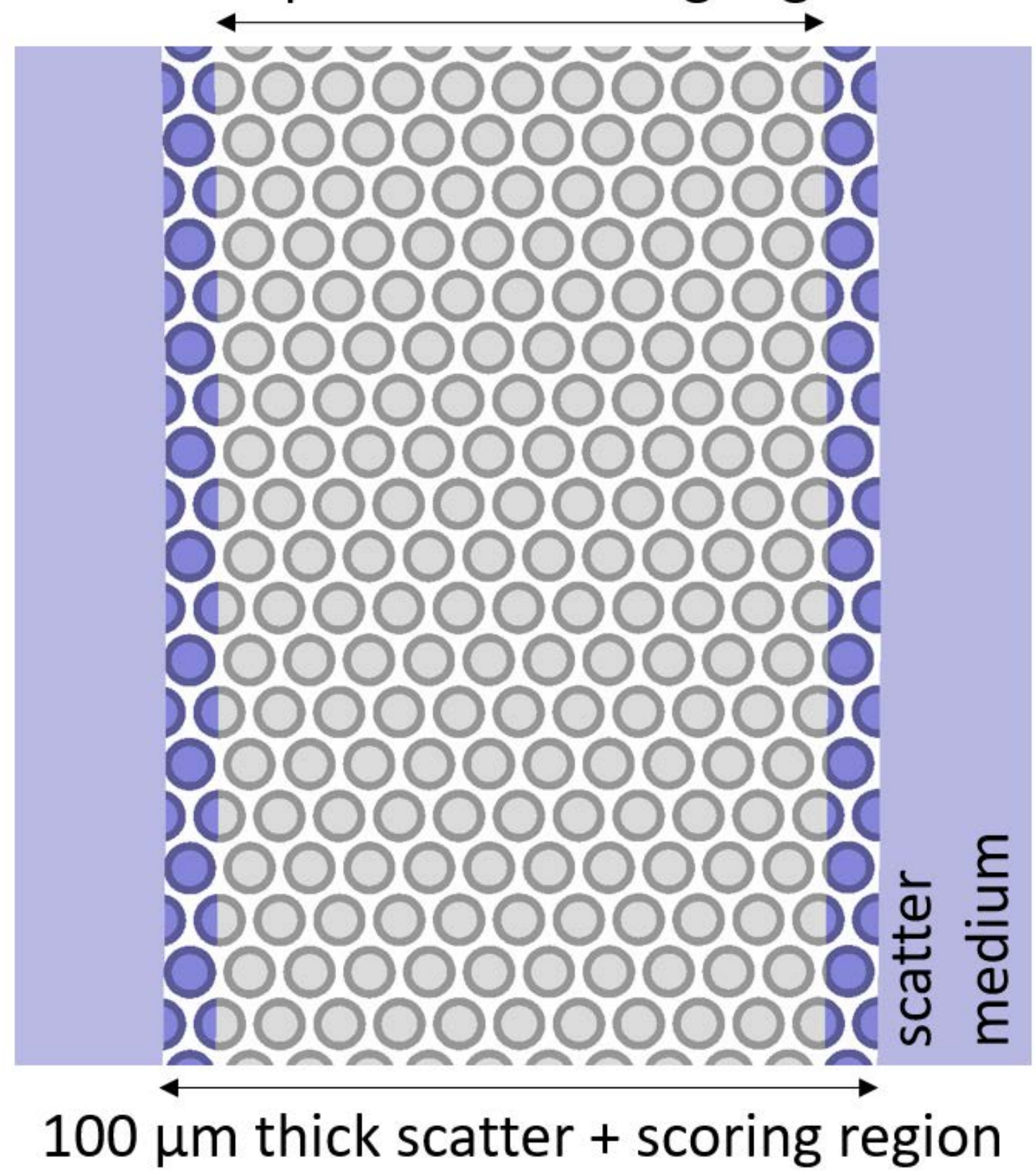

Figure 5.3: Microscopic scoring region (100 $\mu \mathrm{m}$ thick spherical shell) containing cells with GNPs in the perinuclear configuration generated using EGSnrc. Grey nucleus and cytoplasm are the scoring volumes, blue regions are the buffer. The shell is embedded in a single, homogeneous scatter medium with matching average elemental composition. 
Table 5.1 lists variations when fluctuating cell/nucleus size and target/neighbour gold concentration for a "peri" configuration cell in tissue with a concentration of $20 \mathrm{mg} / \mathrm{g}$ and shows a sample calculation of $\% \Delta_{\mathrm{DEF}}^{+}$and $\% \Delta_{\mathrm{DEF}}^{-}$. Scenarios for which the gold concentration or cell size would not allow for an endosome that fits within the cytoplasm are omitted from the statistical populations. A set of $\% \Delta_{\mathrm{DEF}}^{+}$and $\% \Delta_{\mathrm{DEF}}^{-}$values for parameters investigated in this chapter are compiled in tables 5.2 and 5.3, respectively.

Both $\% \Delta_{\mathrm{DEF}}^{+}$and $\% \Delta_{\mathrm{DEF}}^{-}$are largest for the "peri" configuration, with $\% \Delta_{\mathrm{DEF}}$ values for nucleus typically larger than those for cytoplasm. For the "1-endo" and "4-endo" configurations, $\% \Delta_{\mathrm{DEF}}$ values are at or below $10 \%$ for all but a few cases. The largest $\% \Delta_{\mathrm{DEF}}$ values occur at energies for which DEFs are highest, i.e., 20, 30, and $90 \mathrm{keV}$ (shown in figure 4.4 in chapter 4 ), and can drop to a few percent at energies for which DEFs are close to unity. In cases with a $\% \Delta_{\mathrm{DEF}}^{+}$of $10 \%$ or higher, the $\% \Delta_{\mathrm{DEF}}^{+}$is always larger than or equal to the equivalent $\% \Delta_{\mathrm{DEF}}^{-}$, often larger by a factor of 2 or higher; this follows from the ranges found in nucleus and cytoplasm DEFs (n,cDEFs) in figure 4.6 in chapter 4 . The largest overall variation in DEFs is for nDEFs in the "peri" configuration from a $90 \mathrm{keV}$ beam, for which the $\% \Delta_{\mathrm{DEF}}^{+}$is $76 \%$. The smallest variation in DEF is also found for nDEFs from a $90 \mathrm{keV}$ beam but for the "4-endo" configuration, for which $\% \Delta_{\mathrm{DEF}}^{-}$is $4 \%$.

Table 5.1: $\% \Delta_{\text {DEF }}^{+}$(top) and $\% \Delta_{\text {DEF }}^{-}$(bottom) variation for a "peri" configuration reference cell in $20 \mathrm{mg} / \mathrm{g}$ concentration as the quadrature sum of cell/nucleus size and target/neighbour gold fluctuation.

\begin{tabular}{|c|c|c|c|c|c|c|}
\hline & \multirow{2}{*}{ fluctuation } & \multicolumn{5}{|c|}{ energy $(\mathrm{keV})$} \\
\hline & & 20 & 30 & 50 & 70 & 90 \\
\hline \multirow{3}{*}{ increase } & cell size & $22 \%$ & $25 \%$ & $8 \%$ & $7 \%$ & $21 \%$ \\
\hline & gold conc. & $18 \%$ & $16 \%$ & $7 \%$ & $2 \%$ & $11 \%$ \\
\hline & $\% \Delta_{\mathrm{DEF}}^{+}$ & $29 \%$ & $29 \%$ & $11 \%$ & $7 \%$ & $24 \%$ \\
\hline \multirow{3}{*}{ decrease } & cell size & $29 \%$ & $40 \%$ & $11 \%$ & $7 \%$ & $51 \%$ \\
\hline & gold conc. & $14 \%$ & $14 \%$ & $2 \%$ & $6 \%$ & $13 \%$ \\
\hline & $\% \Delta_{\text {DEF }}^{-}$ & $32 \%$ & $42 \%$ & $11 \%$ & $9 \%$ & $52 \%$ \\
\hline
\end{tabular}




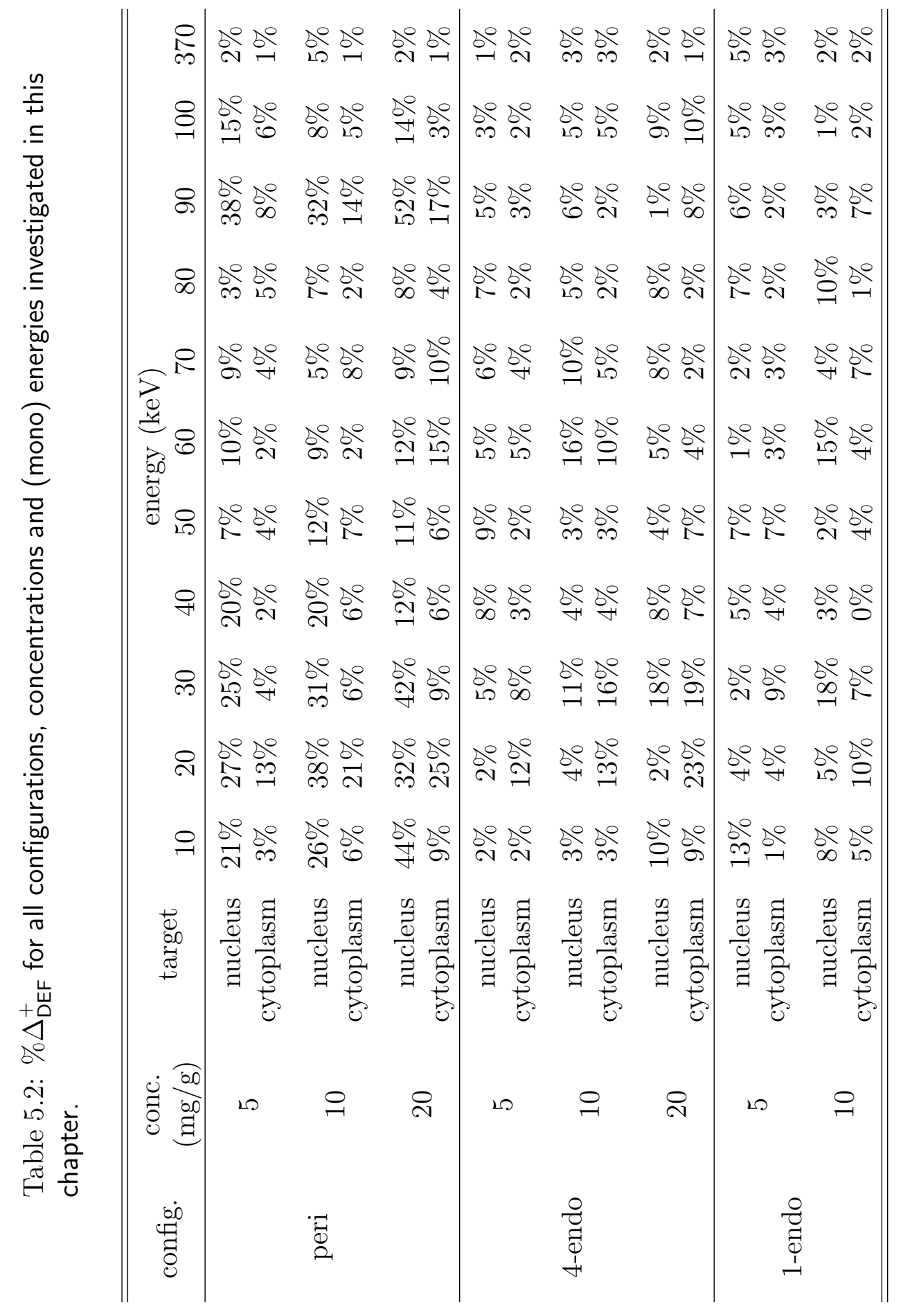




\begin{tabular}{|c|c|c|c|c|c|c|c|c|c|}
\hline & $\underset{\infty}{R}$ & 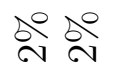 & ล̊ำ & ô & ลิ ลิ ลิ & ลั ลे & se $^{0}$ & ลั & ํํำ \\
\hline.$\subseteq$ & $\underset{\Xi}{\S}$ & do & $\begin{array}{ll}00 \\
10\end{array}$ & $\begin{array}{l}\text { ô } \\
\text { के } \\
\text { ปे }\end{array}$ & ถิำ & do & 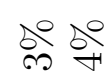 & 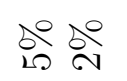 & $\sum_{\rightarrow}^{0}$ \\
\hline$\cdot \frac{00}{\tilde{n}}$ & \& & 起 & ปી & 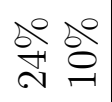 & de & 워 & के ది & के దे & 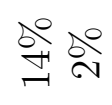 \\
\hline$\stackrel{. \subseteq}{y}$ & 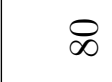 & ลे ลे & ถั่ & $\begin{array}{ll}0 \\
\infty\end{array}$ & 암 ลำ & îㅇำ & ลั ลำ & bํํㅁ & bo bo \\
\hline $\begin{array}{l}\overline{00} \\
\overline{\frac{d}{\alpha}} \\
\overline{\frac{1}{\alpha}}\end{array}$ & $\Omega^{R}$ & 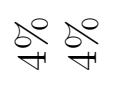 & मू & $\begin{array}{ll}0 \\
1\end{array}$ & $\begin{array}{ll}\text { se } & 0 \\
\text { के }\end{array}$ & 瓷 & ลे ลे & 登 & 암 웜 \\
\hline วิ & $\stackrel{\mathscr{C}}{=} 8$ & 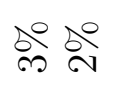 & $\begin{array}{l}00 \\
0 \\
7\end{array}$ & $\begin{array}{ll}0 & 0 \\
\infty & 0 \\
\infty & 1\end{array}$ & $\begin{array}{ll}2 & 0 \\
\infty & 0\end{array}$ & de & 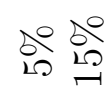 & $\begin{array}{lll} & 0 \\
10 & 0 \\
10\end{array}$ & $\begin{array}{l}\text { so } \\
\infty\end{array}$ \\
\hline$\underset{\frac{T}{\sigma}}{\stackrel{T}{\sigma}}$ & $\underset{0}{0}$ & 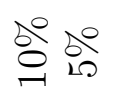 & $\begin{array}{l}0^{0} \\
\text { m } \\
\rightarrow\end{array}$ & 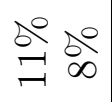 & $\begin{array}{ll}0 & 0 \\
\text { m } & 10\end{array}$ & $\begin{array}{ll}\text { do } \\
\text { in }\end{array}$ & $\begin{array}{l}\delta^{0} \\
b^{\circ}\end{array}$ & 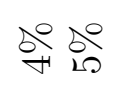 & $\begin{array}{ll} & \text { se } \\
\text { in } & \text { in }\end{array}$ \\
\hline 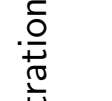 & $\stackrel{\bigodot}{\not}$ & $\begin{array}{ll}0 & 0 \\
10 & \infty\end{array}$ & & $\begin{array}{ll}D_{0}^{0} & 0 \\
\infty & 0 \\
\text { N } & 1\end{array}$ & $\begin{array}{l}30 \\
\text { in }\end{array}$ & 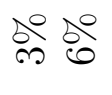 & ถి & be & 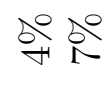 \\
\hline 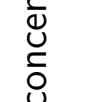 & $\stackrel{\curvearrowright}{\circ}$ & 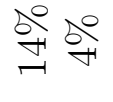 & 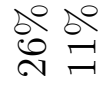 & 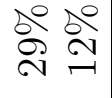 & S̊ำ & 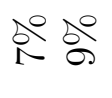 & do & $\begin{array}{ll}80 & 0 \\
10 & 0 \\
-1 & 0\end{array}$ & 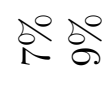 \\
\hline 뭉 & $\stackrel{\curvearrowright}{\curvearrowright}$ & $\begin{array}{l}\text { Do } \\
\text { ô } \\
\text { b }\end{array}$ & $\begin{array}{ll}\text { so } \\
\text { 금 }\end{array}$ & 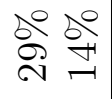 & $\begin{array}{ll}\text { de } \\
1 \\
1\end{array}$ & 然题 & se & $\begin{array}{ll}0 & 0 \\
\text { m } & 10\end{array}$ & 㲾 \\
\hline 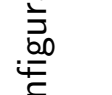 & $\stackrel{\varrho}{\varrho}$ & 良 & $\begin{array}{l}\delta^{0} \text { o } \\
\text { m } 0\end{array}$ & $\begin{array}{ll}0 & 0 \\
\infty & 0 \\
\infty & 0 \\
-1 & \end{array}$ & 方 & 盗 & $\begin{array}{l}50 \\
\text { in } \\
0\end{array}$ & ล̊ำ & $\stackrel{2}{a}$ \\
\hline 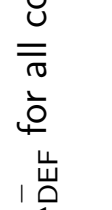 & 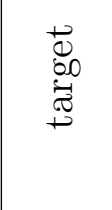 & 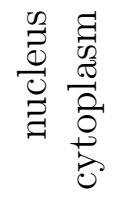 & 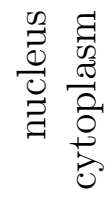 & 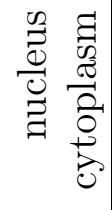 & 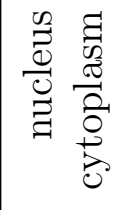 & 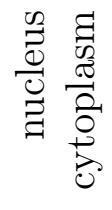 & 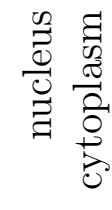 & 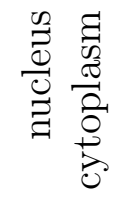 & 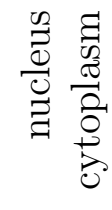 \\
\hline$\ddot{\dot{\theta}}$ & 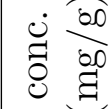 & 10 & $\underset{-}{\varrho}$ & ฉి & 10 & $\stackrel{ }{-}$ & $\stackrel{\curvearrowright}{\curvearrowright}$ & ס & $\stackrel{ }{-}$ \\
\hline & 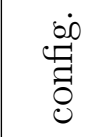 & & $\overrightarrow{0}$ & & & 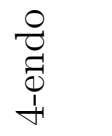 & & $\begin{array}{l}0 \\
\stackrel{0}{0} \\
\stackrel{1}{1} \\
\end{array}$ & \\
\hline
\end{tabular}




\subsubsection{DEF in a tumour with constant macroscopic gold concen- tration}

Figure 5.4 shows nucleus and cytoplasm DEF (nDEF and cDEF, respectively; n,cDEF, collectively) as a function of depth at the highest gold concentrations investigated $(20 \mathrm{mg} / \mathrm{g})$ for monoenergetic 20 and $90 \mathrm{keV}$ photons and an ${ }^{125} \mathrm{I}$ source. The coloured lines represent either "peri" or "4-endo" configurations with the corresponding shaded regions representing the variation in DEFs expected based on the values calculated in section 5.3.1. The shaded region for ${ }^{125} \mathrm{I}$ (average energy $27.5 \mathrm{keV}$ ) was calculated using the $30 \mathrm{keV}$ variations. The black line represents dose to tissue throughout the sensitive area of the microscopic scoring cavity with $25 \mathrm{~nm}$ radius GNPs spread out evenly in a hexagonal lattice geometry, called the dispersed model. DEFs in the dispersed $25 \mathrm{~nm}$ GNP model lie between the 10 and $50 \mathrm{~nm}$ radius GNPs investigated previously in chapter 3. Though DEFs are above unity for energies above $30 \mathrm{keV}$, DEFs $<1$ occur for both ${ }^{125} \mathrm{I}$ and $20 \mathrm{keV}$ energies with an nDEF below one as shallow as $3 \mathrm{~mm}$ depth for the "4-endo" configuration at $20 \mathrm{keV}$. Trends are similar to those in chapter 3 .

The highest nDEF occurs for the "peri" configuration in ${ }^{125} \mathrm{I}$, ranging $4.7-9.3$ at the surface and 0.9-1.7 at $3 \mathrm{~cm}$ depth. Though nDEFs at $20 \mathrm{keV}$ are close to those at ${ }^{125} \mathrm{I}$ near the surface (ranging 3.9-7.4), DEFs reduce markedly more as a function of depth for $20 \mathrm{keV}$ energies, dropping to 0.05-0.09 after $3 \mathrm{~cm}$. nDEFs in cells with "4-endo" configurations do not vary much from the nominal values. At $20 \mathrm{keV}$, the "4-endo" configuration nDEFs are 1.52-1.68 near the surface and fall to $\sim 0.05$ after $3 \mathrm{~cm}$. The change in cDEF as a function of energy is similar to the nDEF trends, though "peri" cDEFs are the same or lower and "4-endo" cDEFs are the same or higher than their equivalent nDEFs at the same energy. For all cases, the largest expected "4-endo" n,cDEFs are below the lowest expected "peri" n,cDEFs. 
The above energies were chosen as representative of general trends. The ${ }^{131} \mathrm{Cs}$ and ${ }^{103} \mathrm{Pd}$, as well as $30 \mathrm{keV}$, results were very close to the ${ }^{125} \mathrm{I} \mathrm{n}, \mathrm{cDEFs}$. The change in DEFs with energy on a macroscopic scale follow the results in chapter 3 and on a microscopic scale follow the results in chapter 4 . The interplay between them is not drastic for energies at or between 40 and $80 \mathrm{keV}$ or over $90 \mathrm{keV}$.

Figure 5.5 shows n,cDEFs for a $20 \mathrm{keV}$ source energy at concentrations of 5 and $10 \mathrm{mg} / \mathrm{g}$. The "peri" configuration has the highest nDEFs and cDEFs, as in the $20 \mathrm{mg} / \mathrm{g}$ case. Though the initial DEFs at 5 and $10 \mathrm{mg} / \mathrm{g}$ are lower than the analogous DEFs at $20 \mathrm{mg} / \mathrm{g}$, they become relatively larger than their analogues at depths between $0.2-1.0 \mathrm{~cm}$ due to the lower gold content in the scatter media. The "1-endo" and "4-endo" configurations nDEFs agree within uncertainty at all depths; when comparing cDEFs of the two configurations, the "1endo" configuration cDEFs are lower than the "4-endo" cDEFs at the same depth largely due to the increased absorption in GNPs of the larger endosome. Though the absolute values vary, the change in DEF as a function of depth in Figures 5.4 and 5.5 follows similar trends to those with dispersed GNPs in a cubic lattice in section 3.3.1 (chapter 3).

Figure 5.6 gives the n,cDEF over dispersed DEF ratios as a function of depth (using results from figure 5.4). The ratio at $90 \mathrm{keV}$ is constant (within uncertainty) with depth, showing that the (relative) difference in DEFs between the models does not vary with depth. In the $20 \mathrm{keV}$ case however, the 4 different ratios all trend upward (7-16\% increases) for the first $\mathrm{cm}$ or so before plateauing and getting very noisy due to the lack of photons penetrating deep into the gold-tissue mixture. A similar, albeit smaller, trend in ratios over the first $\mathrm{cm}$ is somewhat apparent in the ${ }^{125} \mathrm{I}$ case. 

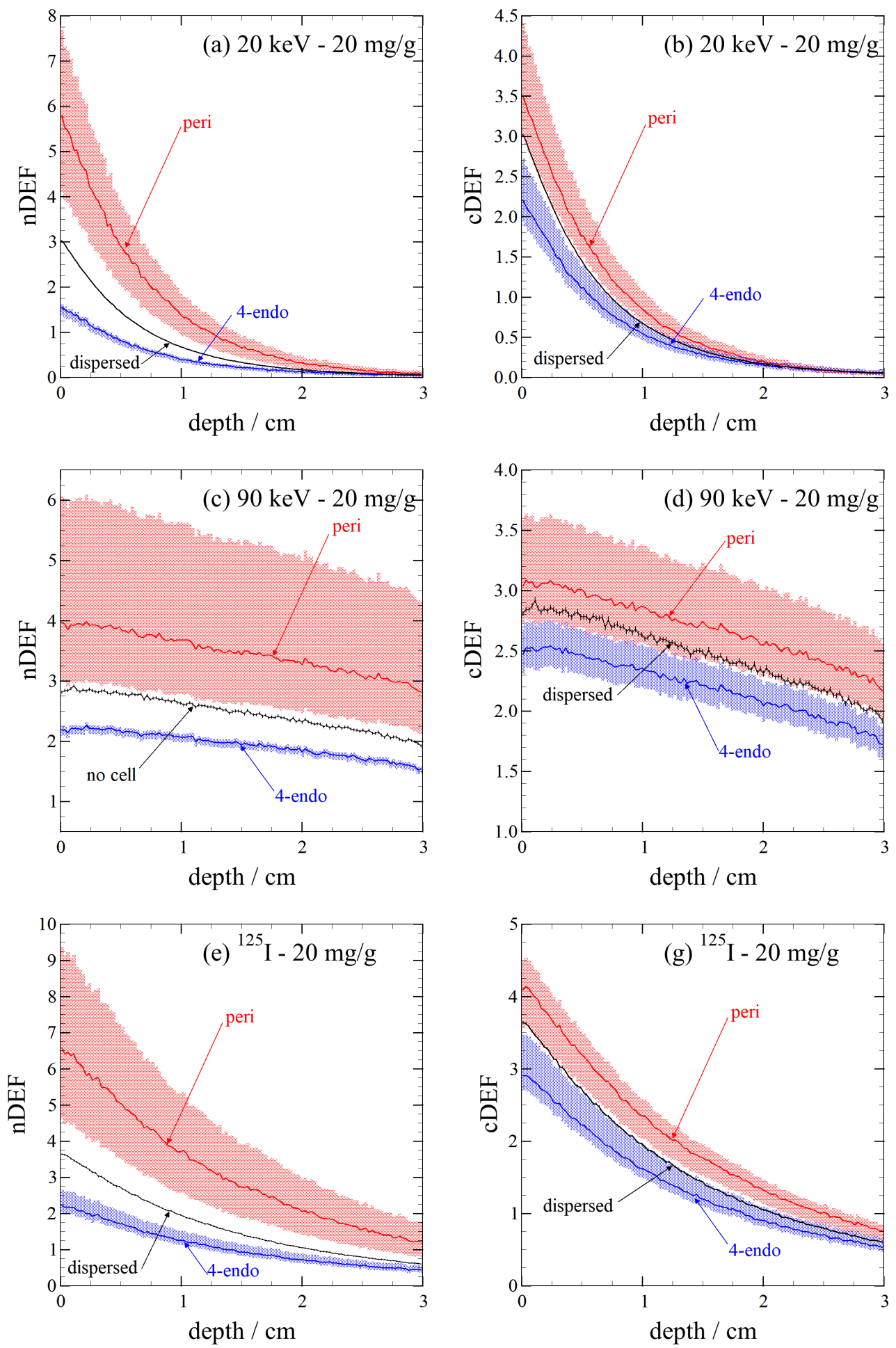

Figure 5.4: Dose enhancement factor of the nucleus (left) or cytoplasm (right) as a function of depth for perinuclear (red) and four endosome (blue) configurations for $20 \mathrm{keV}$ (top), $90 \mathrm{keV}$ (middle), and ${ }^{125} \mathrm{I}$ (bottom) source energies for a gold concentration of $20 \mathrm{mg} / \mathrm{g}$. Shaded regions represent $\% \Delta_{\text {DEF }}$ (tables 5.3 and 5.2) and the solid black line is tissue DEF to the scoring cavity when only modelling dispersed GNPs. 

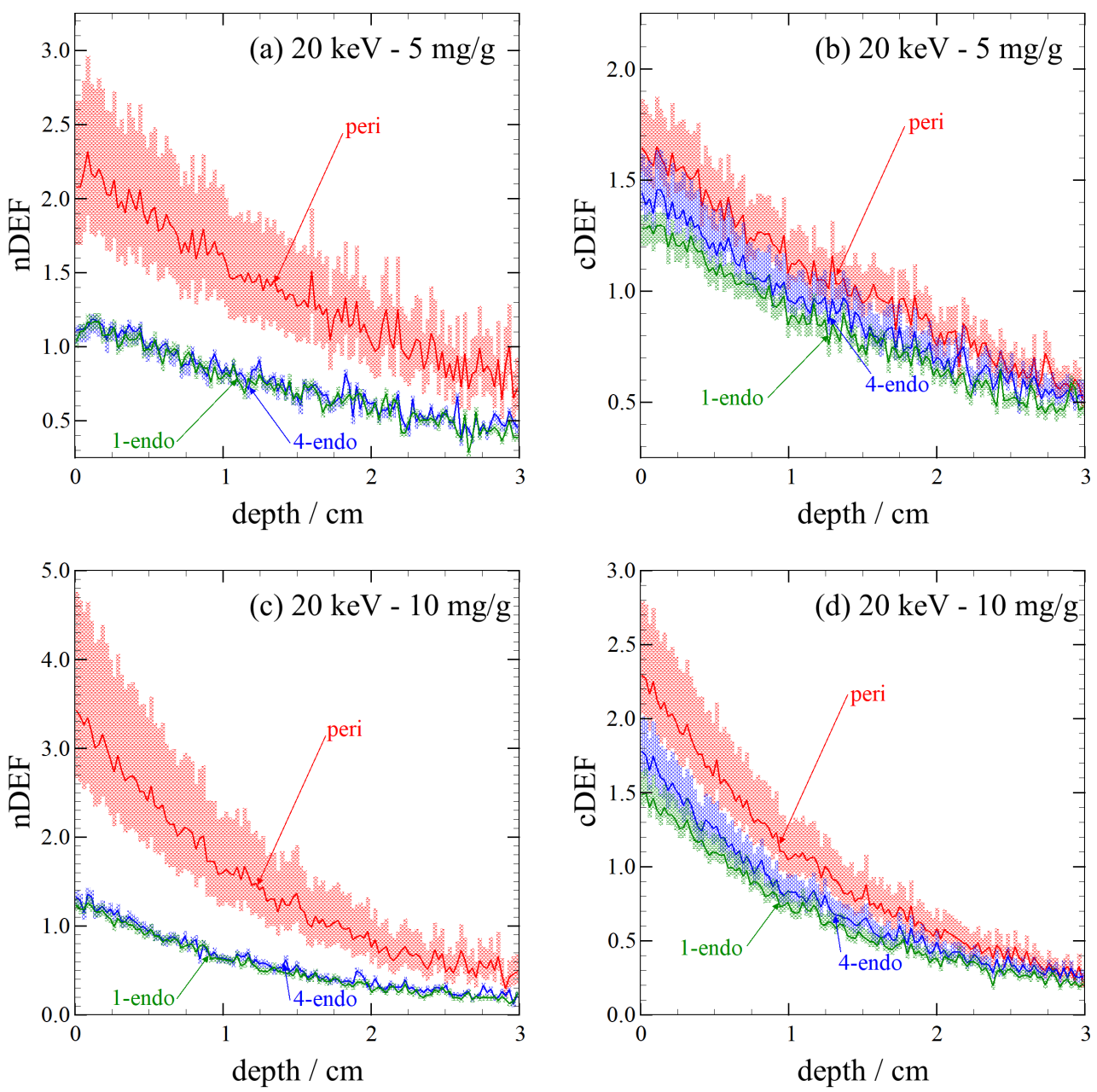

Figure 5.5: Dose enhancement factor of the nucleus (left) or cytoplasm (right) as a function of depth for perinuclear (red), four endosome (blue) and single endosome (green) configurations for $20 \mathrm{keV}$ photons for gold concentrations of 5 (top) and $10 \mathrm{mg} / \mathrm{g}$ (bottom). Shaded regions represent $\% \Delta_{\mathrm{DEF}}($ tables 5.3 and 5.2). 

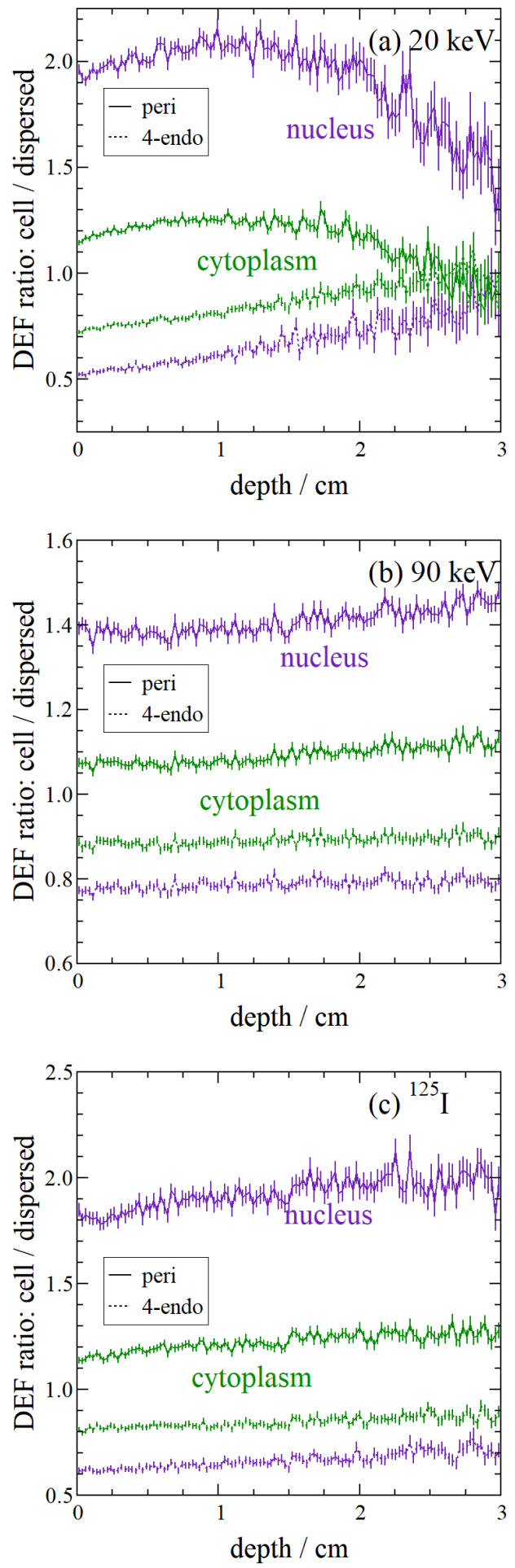

Figure 5.6: DEF ratio when scoring dose to cell sub-compartments ( $n, c D E F$; coloured lines in figure 5.4) over dose to tissue containing evenly dispersed GNPs (black lines in figure 5.4) in a cylinder with an average gold concentration of $20 \mathrm{mg} / \mathrm{g}$ for (a) $20 \mathrm{keV}$, (b) $90 \mathrm{keV}$, and (c) ${ }^{125}$ I sources. 


\subsubsection{DEF in a tumour with varying gold concentration in a brachytherapy scenario}

Figure 5.7 shows n,cDEF of the three cell GNP configurations ("peri", "1-endo", and "4endo") and dispersed model DEF as a function of radius, for $20 \mathrm{keV}, 90 \mathrm{keV}$, and ${ }^{125}$ I energies in a sphere containing gold eluted from the center over 33 days, with dose calculated on day 33. Analogous DEFs for 5 and 59 days of elution are plotted in figure 5.8. Results for 1 and 200 days are omitted as in the first case the local dose enhancement is very small and in the latter the attenuation from the gold near the center is very large. The 33 day $10 \mathrm{~nm}$ radius GNP results for the 6711 seed from figure 3.5a (chapter 3) are close to agreement with the results computed for the dispersed $25 \mathrm{~nm}$ radius GNPs model with ${ }^{125} \mathrm{I}$ in this section.

Looking at figure 5.7, all of the GNP configuration models (and the dispersed model) converge to DEFs of $\sim 0.86, \sim 0.99$, and $\sim 0.94$ at radii $>1 \mathrm{~cm}$ for $20 \mathrm{keV}, 90 \mathrm{keV}$, and

${ }^{125} \mathrm{I}$, respectively. This convergence occurs in the regions where the gold concentration is essentially zero and the choice of model in the microscopic scoring cavity no longer matters, as it is all modelled as tissue; the convergence occurs at a lower radius for 5 days and at a larger radius for 59 days, as expected based on gold concentration. Thus, the choice of model in the microscopic scoring cavity attenuates the primary photons equally, verifying that the models are equivalent on the macroscopic scale. For the point nearest the centre $(\mathrm{r}=0.05 \mathrm{~cm})$, the highest $\mathrm{nDEF}(2.9)$ and $\mathrm{cDEF}(2.2)$ is found for the "peri" configuration for the ${ }^{125}$ I spectrum and the lowest central nDEF (1.3) and cDEF (1.4) is for the "1-endo" and "4-endo", respectively, for $90 \mathrm{keV}$ photons.

Figure 5.9 shows the $\mathrm{n}, \mathrm{cDEF}$ over dispersed DEF ratios as a function of radius after 33 days for different energies (figure 5.10 shows the ratios after 5 and 59 days). In all cases, the DEF ratios converge to 1 at depths where the gold concentration is zero. The initial ratios range from 0.7 (endosome configuration nDEF ratios for $20 \mathrm{keV}$ photons after 59 days) to 
1.5 ("peri" configuration nDEF ratios for $20 \mathrm{keV}$ photons after 59 days), but all the ratios themselves approach unity as a function of depth. For 33 and 59 days, the DEF ratio versus depth trend appears to follow a first or second order polynomial drop-off until convergence. At 5 days, the ratios appear more nuanced; e.g., the "peri" configuration cDEF for $20 \mathrm{keV}$ photons initially trends away from unity for the first $0.2 \mathrm{~cm}$ until returning to unity at $0.4 \mathrm{~cm}$.

\subsection{Discussion}

The HetMS simulations of GNPT determined cellular DEFs (n,cDEFs) across a cm-scale phantom in a single simulation, characterizing DEF as a function of depth. This HetMS implementation allows for the exploration of a large parameter space (both macro- and microscopic parameters) which would otherwise not be computationally feasible, i.e., source energy, GNP configuration within the cell, depth in the tumour. The n,cDEFs computed in the HetMS cylinders varied significantly from the DEFs to tissue with GNPs evenly dispersed throughout it, indicating a need to calculate n,cDEFs in GNPT research, treatment planning, and dose estimation. The variation of $n, c D E F s$ with biological fluctuations in cell/nucleus size and target/neighbouring cell gold concentration are computed and compiled into reference tables of expected variations in DEFs $\left(\% \Delta_{\mathrm{DEF}}\right)$. The $\% \Delta_{\mathrm{DEF}}^{-}$values, the lower bound of the cell DEF variations, are particularly of interest as they represent the lowest DEFs expected across a population of cells (as low as $29 \%$ in some cases) which are relevant for both development research and clinical applications of GNPT. 

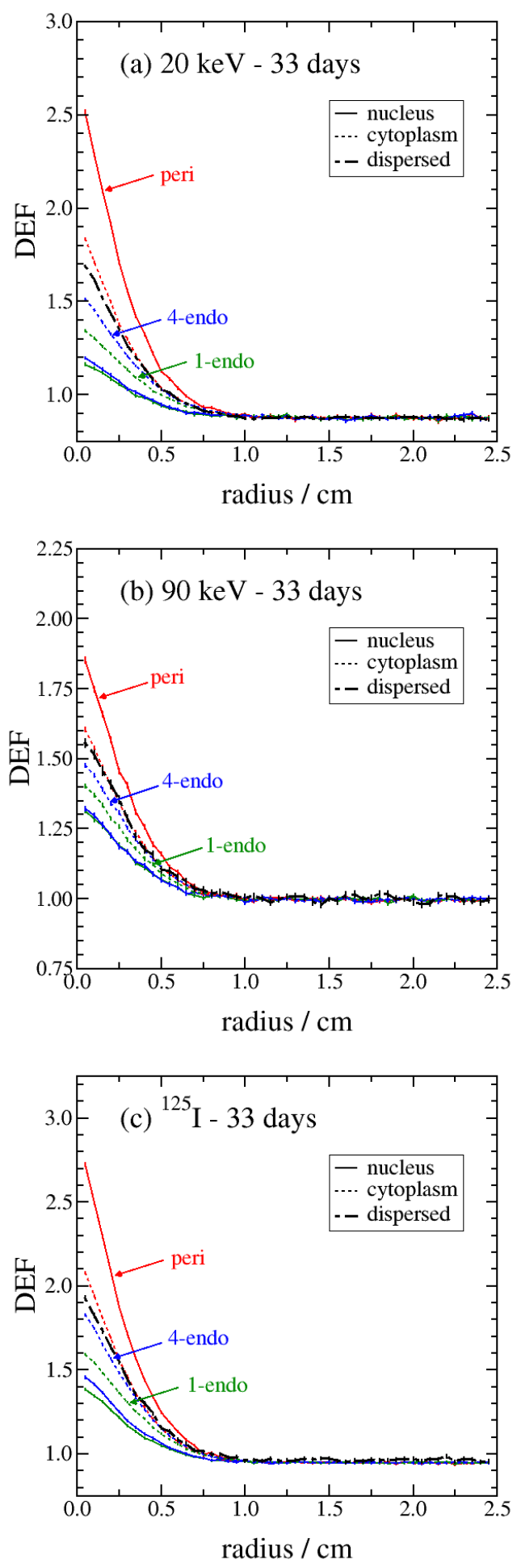

Figure 5.7: n,cDEF as a function of radius in a sphere after 33 days of elution containing a (a) $20 \mathrm{keV}$, (b) $90 \mathrm{keV}$, and (c) ${ }^{125}$ I point source in its centre for "peri", "1-endo", and "4-endo" configurations. Solid black line is tissue DEF to the scoring cavity when modelling only dispersed GNPs. 

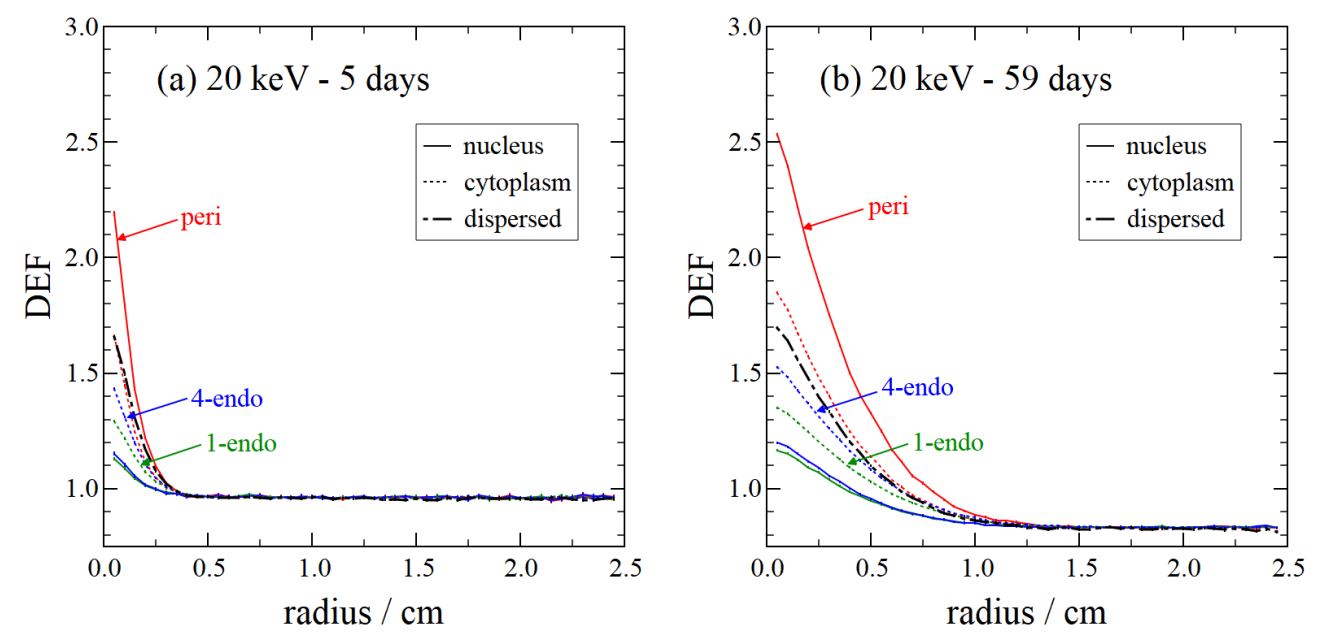

Figure 5.8: n,cDEF as a function of radius in a sphere after (a) 5 and (b) 59 days of elution containing a $20 \mathrm{keV}$ monoenergetic point source in its centre for "peri", "1-endo", and "4-endo" configurations. Solid black line is tissue DEF to the scoring cavity when modelling only dispersed GNPs.

The combined $\% \Delta_{\mathrm{DEF}}$ values (tables 5.2 and 5.3) computed for 5,10 and $20 \mathrm{mg} / \mathrm{g}$ concentrations and 10-370 keV energies provide an expected variation in DEFs in GNPT for "peri", "1-endo", and "4-endo" configurations to help account for many of the biological considerations of GNPT on a cellular level. ${ }^{109,115,118}$ Although a $20 \%$ fluctuation in gold concentration could be a large underestimate of the actual difference in cell-to-cell GNP uptake, ${ }^{40}$ the $20 \%$ variation was chosen to provide an (ideal) representative value for proposed GNPT deliveries/treatments in the literature. ${ }^{23,33,63,67}$ These factors can be used in a GNPT scenario to provide a range of expected nDEFs and cDEFs based on calculations of reference DEFs, thus providing an estimate of n,cDEFs over an entire population of tumour cells as opposed to a single nominal value. Figure 5.4 shows the ranges of n,cDEFs expected in a simple GNPT scenario as a function of depth for different energy photons, demonstrating the large differences (100\%) in n,cDEFs achieved over a cell population at the same position.

The nDEFs and cDEFs at the surface of the cylinder (figure 5.4) at $20 \mathrm{keV}$ are within a few percent of the equivalent reference n,cDEFs in figure 4.4 (chapter 4 ). The surface DEFs at $90 \mathrm{keV}$, on the other hand, are markedly larger in the cylinder simulation; this increase is due to the increased number of scattered photons in the much larger volume entering the 

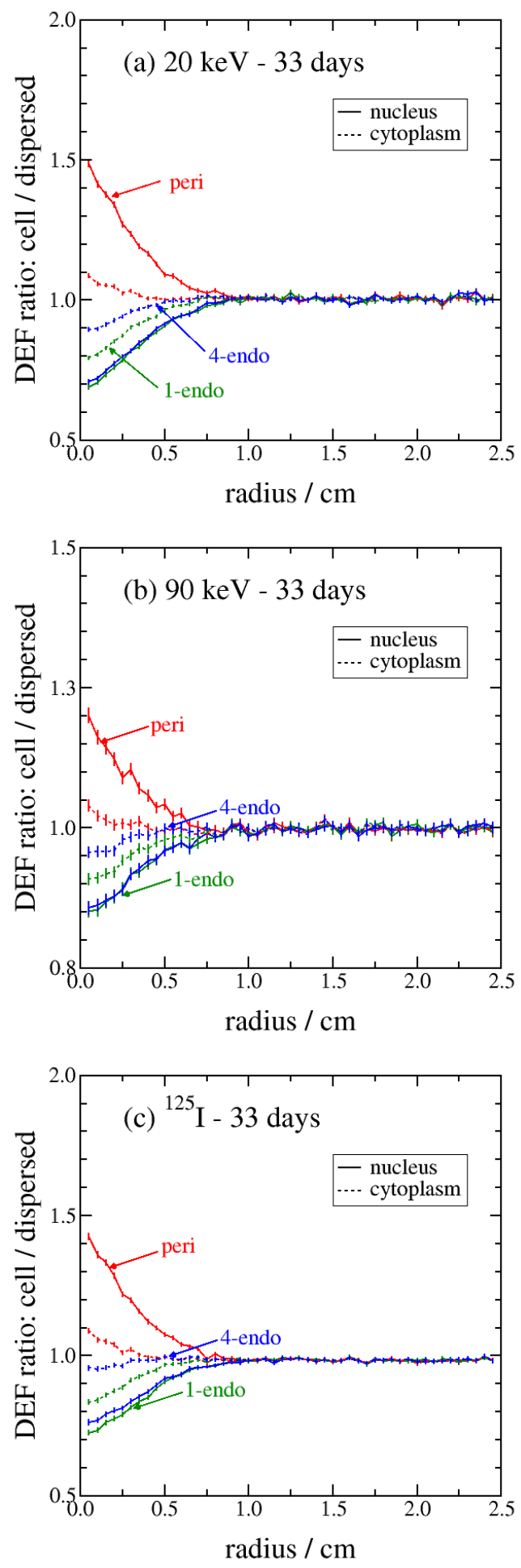

Figure 5.9: DEF ratio when scoring dose to cell sub-compartments ( $n, c D E F$; coloured lines in figure 5.7) over dose to tissue media containing evenly dispersed GNPs (black lines in figure 5.7) in a gold-filled sphere after 33 days of elution for (a) $20 \mathrm{keV}$, (b) $90 \mathrm{keV}$, and (c) ${ }^{125}$ I sources. 

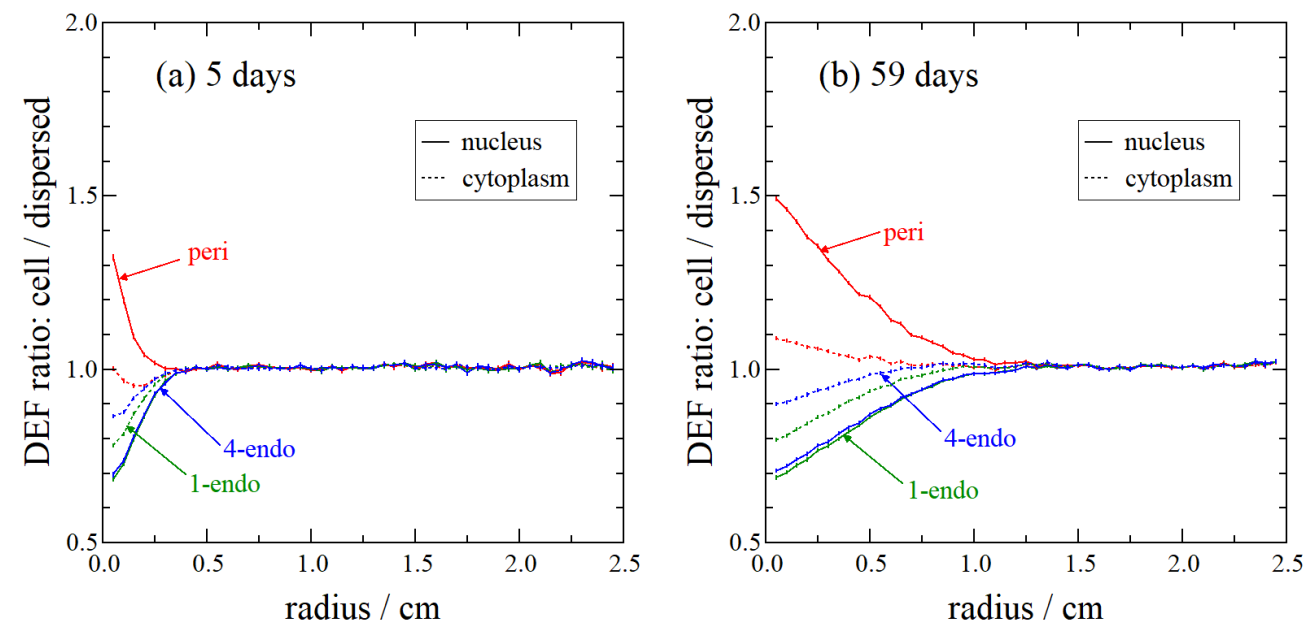

Figure 5.10: DEF ratio when scoring dose to cell sub-compartments ( $n, c D E F$; coloured lines in figure 5.8) over dose to tissue media containing evenly dispersed GNPs (black lines in figure 5.8) in a gold-filled sphere after (a) 5 or (b) 59 days of elution for a $20 \mathrm{keV}$ monoenergetic sources.

microscopic scoring cavity. In figure 5.4, the DEFs decrease with depth and can fall below unity, similar to the cylinder results from chapter 3 (figure 3.3). DEFs below one for ${ }^{125}$ I were also found in the homogeneous mixture DEF values calculated in the prostate simulations of Brivio et $a l,{ }^{23}$ with DEFs as low as 0.7 in parts of the treatment volume. The DEFs of the model with GNPs dispersed evenly in tissue (in agreement with the analogous results in chapter 3) lie between the "peri" and "4-endo" DEFs in all cases. The dispersed DEFs is larger than the endosome n,cDEFs due to the GNPs being spread evenly throughout the scoring volume, minimizing photoelectron absorption in neighbour GNPs. The dispersed DEFs are smaller than "peri" n,cDEFs, because there is also less photoelectron absorption in gold in the "peri" configuration, but, due to the thinness of the gold-containing region, the GNPs are positioned very close to the scoring volumes.

Figure 5.6 demonstrates a fairly constant DEF ratio of cell/dispersed models with depth at $90 \mathrm{keV}$ source energy. Thus, it is possible to apply a fixed factor converting dispersed DEF to nDEF and cDEF for different cell configurations. At lower energies, this factor varies by roughly $16 \%$ for $20 \mathrm{keV}$ energies, but perhaps several factors at choice depths could be used instead. Following the lead of Koger and Kirkby, ${ }^{53}$ who calculate conversion factors to 
determine tissue DEF in a dispersed GNP model using DEF to a homogeneous gold-tissue mixture, a similar approach can be taken with the cell models herein. Conversion factors that yield $\mathrm{nDEF}$ and $\mathrm{cDEF}$ from a homogeneous gold-tissue mixture DEF for a particular energy, gold concentration, GNP size, and gold configuration could be compiled to enable calculation of nominal cell DEF using a quick (and simple) macroscopic GNPT model ${ }^{49-51}$ for a parameter space matching tables 5.2 and 5.3. Sample tables of conversion factors computed for this purpose can be found in appendix C.

Though the ratio between cell and dispersed GNP models is near constant for the cylinder scenario with a constant macroscopic gold distribution, the ratio between the models for the sphere with a varying gold concentration changes with radius (figure 5.9). The change in ratio is due to the different approach in modelling the gold-containing region and scoring volumes in the cell and dispersed models. In the cell model, the gold-containing region is defined at fixed positions in the cell at a set distance from the scoring volumes (nucleus and cytoplasm), this distance does not vary with gold concentration. Conversely, in the dispersed model, all non-GNP medium is both the scoring volume and the gold-containing region. Thus, at low concentrations, GNPs are spaced much farther apart than in the cell case and the distance between two neighbouring GNPs further increases as concentration decreases. For very low concentrations, the distance between GNPs approaches the range of $10 \mathrm{keV}$ photoelectrons $\left(2.5 \mathrm{\mu m}^{84}\right)$. In this case, a larger fraction of the scoring volume is far from the dose increase around GNPs, ${ }^{54}$ causing a decrease in DEFs beyond the decrease due to the lower number of photoelectrons generated in gold (the primary contributor to DEF decrease in the cell models).

Therefore, in proposed GNPTs with varying gold concentrations, ${ }^{23,33}$ there is a need to accurately model cells (and GNPs within them) at every point of interest within a tumour. Simulations that do not account for the microscopic substructure of GNPs in cells are insufficient to provide accurate DEF estimates, and the relating cell models to simpler 
scoring regions would require a more complex formalism than single conversion factors (such as those made using the cylinder simulations in tables C.1, C.2, and C.3 in the appendix).

Both $\mathrm{nDEF}$ and $\mathrm{cDEF}$ results in all HetMS simulations in this chapter show that the perinuclear configuration is the desired configuration for maximizing dose. This is because GNPs are not clustered, thus generated photoelectrons leave the gold-containing region before absorption in GNPs. ${ }^{111}$ Therefore biological delivery methods that avoid GNPs clustering into a compartment within the cell are desired for higher nucleus and cytoplasm DEFs. In contrast, biological delivery methods that result in GNPs clustering into one or more endosomes can result in $\mathrm{nDEF}$ s ranging over as little as 1-1.5 within $0.25 \mathrm{~mm}$ from the source (figure 5.4a) with DEFs below unity at farther depths; this delivery method (if it achieves the same GNP uptake in cells) is therefore undesirable for any GNPT aiming to increase nucleus dose within even $1 \mathrm{~cm}$ of the treatment source(s).

In section 5.3.1, when calculating DEF ranges with fluctuating gold concentration, it was noted that even when varying the gold concentration up or down $20 \%$ in all the neighbouring cells of the target, there was at most $2 \%$ change in target cell dose. Hence, the contribution of local gold concentration fluctuations to the values in tables 5.2 and 5.3 is primarily dependent on the target cell fluctuations. The lack of sensitivity to neighbour concentration is the motivating factor as to the choice of buffer region size for the HetMS models. The buffer region was chosen to be one cell diameter to ensure all scoring volumes are within fully modelled cells, though they may be neighbouring a partially modelled cell.

Select simulations in section 5.3.2 were repeated with buffer shell sizes up to $50 \mu \mathrm{m}$ in thickness (i.e., the $100 \mu \mathrm{m}$ microscopic cavity sensitive volumes were set to within $50 \mu \mathrm{m}$ radius of the centre) and results were in agreement with the $14.7 \mu \mathrm{m}$ buffer. The use of $1 \mathrm{keV}$ energy transport cutoffs for photons and electrons was also tested with repeat simulations with cutoffs increasing in $125 \mathrm{eV}$ increments (from $1 \mathrm{keV}$ ) up to $2 \mathrm{keV}$ and results agreed within statistical uncertainties for cutoffs up to $1.75 \mathrm{keV}$. 


\subsection{Conclusion}

This chapter presents HetMS simulations of GNPT that account for both the macroscopic effects of introducing gold to a treatment volume and the microscopic effects of GNPs on a cellular level. This represents the culmination of the work in chapters 2-4, HetMS simulations in the modified and validated EGSnrc that would be impossible to perform with traditional MC approaches. Additionally, an expected variation of n,cDEFs accounting for fluctuations in cell (and nucleus) size and local gold concentration is computed to represent the range of DEFs over a population of cells (rather than a single DEF for a nominal cell). For a treatment volume with a macroscopically constant gold distribution, it is possible to use MC simulations of a simple homogeneous treatment volume and use it to calculate an expected nominal cell DEF at fixed depths using conversion factors based on HetMS simulations. With that nominal DEF, using expected variation in DEF also computed in this chapter, one could determine the range of possible DEFs over a population of cells while still only performing the simple, homogeneous simulation. Conversely, for a treatment volume with a varying gold concentration, the conversion factor approach is insufficient. An explicit cell simulation over the entire volume would be required, and this chapter demonstrated the HetMS model is a capable tool for the task. 


\section{Chapter 6}

\section{Conclusion}

The work of Hainfield et al in 2004 using GNPs during irradiation of tumour-bearing mice to increase survival ${ }^{21}$ sparked a large interest in the potential of GNPs in human radiotherapy treatments. Over the next decade, many in vitro and MC studies investigated the effects GNPs have on tissue during irradiation. This thesis began as part of a collaboration, attempting to simulate in vitro cell culture irradiations performed by a research group using EGSnrc. Soon after the work began, the shortcomings of both EGSnrc and many of the MC approaches of other codes in the literature at the time became apparent. The work then pivoted into the thesis as it is now, a work more focused on constructing fast and reliable MC simulations of GNPT in general, with many of the associated trials and tribulations discussed below.

The contiguous gold models of cells simulated in chapter 4 were among the first simulations performed for the thesis. In trying to perform simulations with discrete GNPs, the limitations of the default EGSnrc installation were quickly encountered. The cubic lattice geometry was implemented to avoid the memory limitations of individually modelling billions of GNPs. To ensure EGSnrc and the implemented cubic lattice were reliable for GNP simulations, a large amount of cross-comparison and self-consistency testing was performed. In 
the cross-comparisons with other MC simulations of GNPT, large discrepancies were found between different works, often due to the different MC codes, simulation limitations and approaches to modelling gold containing volumes. The HetMS model for MC simulations of radiation transport was developed to create a framework for reliable and efficient modelling approaches to any GNPT scenario.

To quantify the attenuation effects of gold on a macroscopic scale, effects often ignored in the literature, GNPT simulations using the cubic lattice for GNPs were performed, as detailed in chapter 3. Using HetMS and the cubic lattice, a large parameter space was investigated in a $9.42 \mathrm{~cm}^{3}$ volume. For comparison, the GNP containing volume would have been limited to a $\sim 2 \mathrm{~mm}^{3}$ volume using stock EGSnrc. To achieve the required packing density of GNPs and cells needed for biological modelling, the hexagonal close-packed lattice was developed. With the hexagonal lattice, a large range of parameters, including gold modelling approach, was investigated for a single cell model (chapter 4). The cell model was then used to fill the microscopic scoring cavities of a full GNPT HetMS model, the culmination of many aspects of this work, detailed in chapter 5. Along with those simulations, an expected range of cell DEFs over a population of cells was also tabulated.

Nearing the end of the thesis research, the work in validating EGSnrc had become so extensive that many aspects were pulled together to form the basis of chapter 2. The validation with Geant4-DNA and PENELOPE, as well as the verification through the electron Fano cavity test, demonstrated that EGSnrc can simulate transport reliably at the nanometer scale and score dose accurately at the micrometer scale. The verification work, though performed in the context of GNPT, is distinct from the GNPT investigations in latter chapters to serve as evidence that EGSnrc can be reliable used in the field of microdosimetry. 


\subsection{EGSnrc simulations at short length scales}

EGSnrc has been used to score dose on a microscopic length scale previously in the literature, ${ }^{82,117,119-121}$ with each separate work addressing and contextualizing its use in the field of microdosimetry (to varying degrees). Often used as a standard code for metrology, ${ }^{93}$ as ion chamber response calculations in EGSnrc do not vary with choice of Condensed History parameters ${ }^{74}$ its use in microdosimetry investigations is rare (relative to other codes). This thesis uses EGSnrc extensively to model complex GNPT scenarios on both the macroscopic (whole tumour) scale and the microscopic (individual cells containing GNPs) scale; extensive validation of EGSnrc, along with the geometry and application changes, was performed both for this current work but also for the purpose of validating EGSnrc for any future investigations in microdosimetry.

Chapter 2 shows a sample of the cross-validation performed with EGSnrc for this thesis, ${ }^{53,70}$ chosen because the works investigated a wide range of parameters and clearly defined simulation parameters. Agreement was found with PENELOPE and Geant4-DNA and further verification was performed using the electron Fano cavity test. Fano cavity tests passed for a $2 \mu \mathrm{m}$ nucleus (the smallest cell investigated in this work). However, the lowest bounds of EGSnrc's scoring capabilities were not explored and could prove interesting future research. Further validation was performed in chapters 3 and 5 using a cutoff convergence test. This work shows that the $1 \mathrm{keV}$ cutoff used in EGSnrc (equivalent to a $42 \mathrm{~nm}$ electron range in water ${ }^{122}$ ) does not impact the dose scored to cellular compartments. For scoring cavity sizes below the micron scale, further work would be needed to assess reliability.

The Fano cavity tests not only provided validation for EGSnrc's electron transport reliability, but also for the geometry transport code (e.g., the hexagonal lattice) used in the cell models. The Fano cavity test structure in chapter 2, using the cubic lattice geometry to form recursive boundary conditions can be used to test any geometry regardless of size 
without having to simulate electrons out to large ranges to maintain local charged particle equilibrium. The Fano cavity test, along with other verifications such as cutoff convergence tests, can and should be used to test the reliability of any MC simulation.

\subsection{Further applications of HetMS, in GNPT and be- yond}

The HetMS method introduced in chapter 3, a general methodology for efficient numerical computation of problems with multiple scales, has been used outside the field of medical physics. ${ }^{123}$ For example, it can used for complex models to solve fluid and microfluid problems ${ }^{124}$ (e.g., modelling an adhesive layer at the macro-scale combined with the complex damage evolution taking place at the micro-scale ${ }^{125}$ ) or even in modelling the catalytic ac-

tivity of GNPs. ${ }^{126}$ Although there have been two-stage approaches, ${ }^{16}$ combining macro- and micro-scale simulation components using different techniques (e.g., kernel convolution), the HetMS approach of modelling different levels of detail heterogeneously throughout a single phantom has not been used for MC simulations applied to radiation therapy. The research in this work is the first application of HetMS in this field, allowing for fast and flexible MC simulations of radiotherapy at a cellular level.

The use of HetMS models in MC allows for efficient extraction of microscopic metrics across centimetre scale, but HetMS simulations do require validation to ensure all scales have the same effect on fluence across the phantom (on the macroscopic scale). In this work, the HetMS model was tested and simulations had to be changed appropriately to maintain consistency. For example, one of the first issues encountered was with cm-scale circular source used for the cylinder simulations in chapter 3 ; the source took the square root of the random number generator output (resolution on the order of $10^{-8}$ ) by egs_chamber to determine the radius of the initial particles in the circle. The radius, then, for points

\subsection{FURTHER APPLICATIONS OF HETMS, IN GNPT AND BEYOND}


generated in the circle are discretized on the order of $10^{-4}$, leading to a granularity on the micron scale. This granularity is on the same scale as microscopic cavities used in this work and was causing massive dose fluctuations with change in cell and nucleus radius. Thus, the egs++ high-resolution random number generator had to be used for all HetMS simulations.

One method of validating the HetMS model is by replacing the microscopic scoring cavity with the material used in the macroscopic region and ensuring the primary and secondary scatter fluence is maintained throughout the phantom. This test discovered the need for the lattice geometries to be tilted off the parallel beam axis, as the shadowing effects of aligned GNPs affected the primary photon beam differently within the microscopic scoring regions than without. An alternate (and much slower) test can also be used, where the entire phantom is modelled to the same level of microscopic detail. Dose to the same microscopic scoring cavities in the standard HetMS can then be compared to a fully detailed simulation; this method can only be done for a select few cases, in practice, due to the vast computation times.

The HetMS simulations in this thesis only simulated GNPT on two scales, but the HetMS approach in general does not limit the number of scales. As many scales as needed can be used to maximize simulation efficiency while ensuring accurate scoring. For example, the HetMS method can be used on a detailed CT scan of a patient used to create a MC phantom with a very high-resolution grid. As before, microscopic scoring regions with cellular detail can be placed in the treatment volume to determine cell dose, but to further increase efficiency, the resolution of the phantom can be reduced in regions of the simulation far from the treatment volume.

The HetMS model in MC simulations, introduced in this thesis towards GNPT, is a powerful tool for microdosimetry. It allows for efficient simulations that can extract microscopic (subcellular) dose metrics on a macroscopic (tumour) scale, opening the door to exploring a wide range of parameters that were previously computationally infeasibly. 


\subsection{Considerations for GNPT}

GNPT is very complex, adding many new considerations to dose calculations for typical radiotherapy treatments. Cellular considerations such as GNP size, shape, and delivery method for cells of variable sizes and types. Treatment specific considerations such as the method in which GNPs are introduced to the patient and the distribution of GNPs (over time) in the treatment volume. As well as the interplay between these considerations and the non-GNPT treatment parameters (e.g., dose profiles around brachytherapy seeds in gold-tissue medium).

On a macroscopic scale, adding gold to a tumour can lead to so much photon attenuation through the treatment volume (especially for brachytherapy energies) that even at shallow depths the decrease in cell dose due to the attenuation is larger than the enhancement from the GNPs in the cell. In a brachytherapy scenario, this effect could markedly increase the inhomogeneity of the dose distribution, causing larger cold spots; these larger cold spots could, in turn, lead to treatments sparing more cells in tumours with GNPs than without. On a microscopic scale, GNP configuration in the cell has a large impact on nDEFs and cDEFs. GNPs distributed in a more dispersed configuration throughout the cell are desirable for both nucleus and cytoplasm dose, as the self-absorption of photoelectrons generated in clustered GNP configurations can be avoided. DEF is maximized at energies $10-20 \mathrm{keV}$ above the Land K-edges of gold, depending on cell geometry.

There are also considerations for GNPT that involve a combination of microscopic and macroscopic effects. The variation of cell DEFs with fluctuations in nucleus/cell size and target/neighbour concentration over a population of cells are compiled for a range of treatment parameters. These variations need to be accounted for when planning for GNPT, as a nominal DEF calculated for a single cell will not be representative over all the cells due to the intercellular fluctuations. Further complicating GNPT considerations, the relationship 
between different GNP configuration cell DEF (as it relates to dispersed GNP DEF) can vary with position in the tumour. This difference is especially large in phantoms with heterogeneous gold distributions.

A large parameter space of GNPT dosimetry was explored in chapters 4 and 5: gold concentration, source energy, gold configuration, cell size, nucleus size, target and neighbour cell gold fluctuation, and cell position within the tumour for both nucleus and cytoplasm DEFs. These are all parameters that need to be accounted for in treatment planning of proposed GNPT. This could introduce long, complex computations to clinical treatments (especially if full HetMS simulations of heterogeneous gold distributions are required) and a more heterogeneous dose distribution (with potential cold spots) in the treatment, both of which are undesirable. Compounded with the (relatively) small nDEFs and cDEFs computed for the endosome (the most common) configurations and the possible temporal considerations of gold concentration fluctuating over treatment time, achieving reliable dose increase in GNPT may prove challenging for clinical treatments and treatment plans. Though this work investigated many macro- and micro-scopic factors of GNPT, simulations were performed in (somewhat) limited models. More complex phantoms (with varying tissue composition) and cells (with more subcellular structures) could help push GNPT understanding further, and the HetMS framework presented herein would still prove an excellent tool for such simulations. 


\section{Appendix A}

\section{Cubic lattice implementation}

This section contains the isWhere(), hownear() and howfar() implementations in the egs_cubic_lattice c ++ geometry created for the thesis as well as the closestPoint() function used throughout them. Here, the variable spacing is defined at construction, and is the centre-to-centre distance between two neighbouring subgeometries.

Full geometry class available at https://github.com/MartinMartinov/EGSnrc/ at the time of publication, but a more general, clean implementation will be pushed to the main EGSnrc distribution in the future.

\section{A.1 closestPoint()}

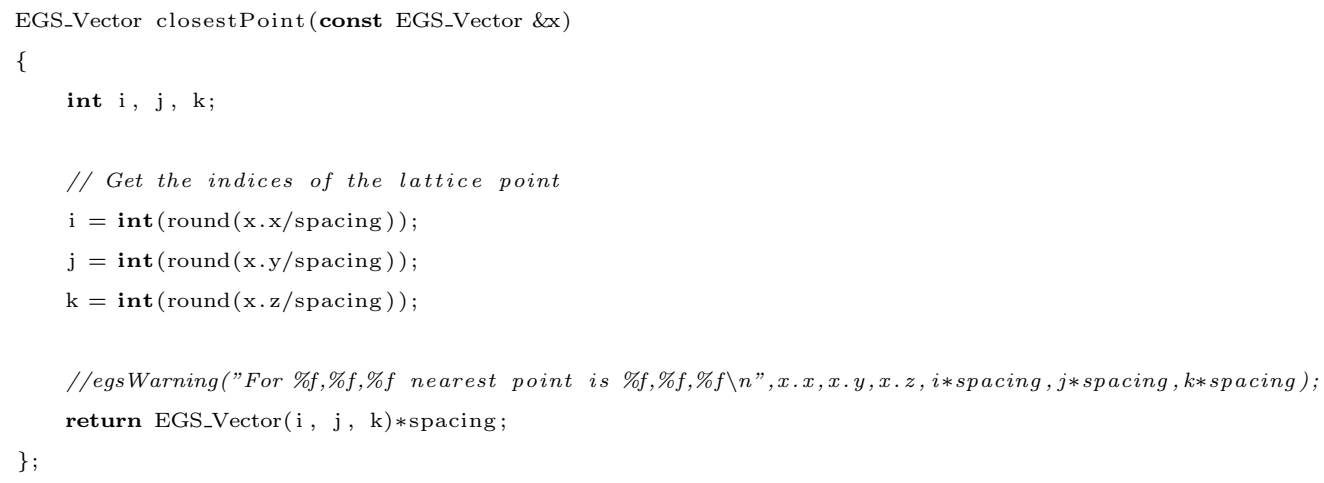




\section{A.2 is Where ()}

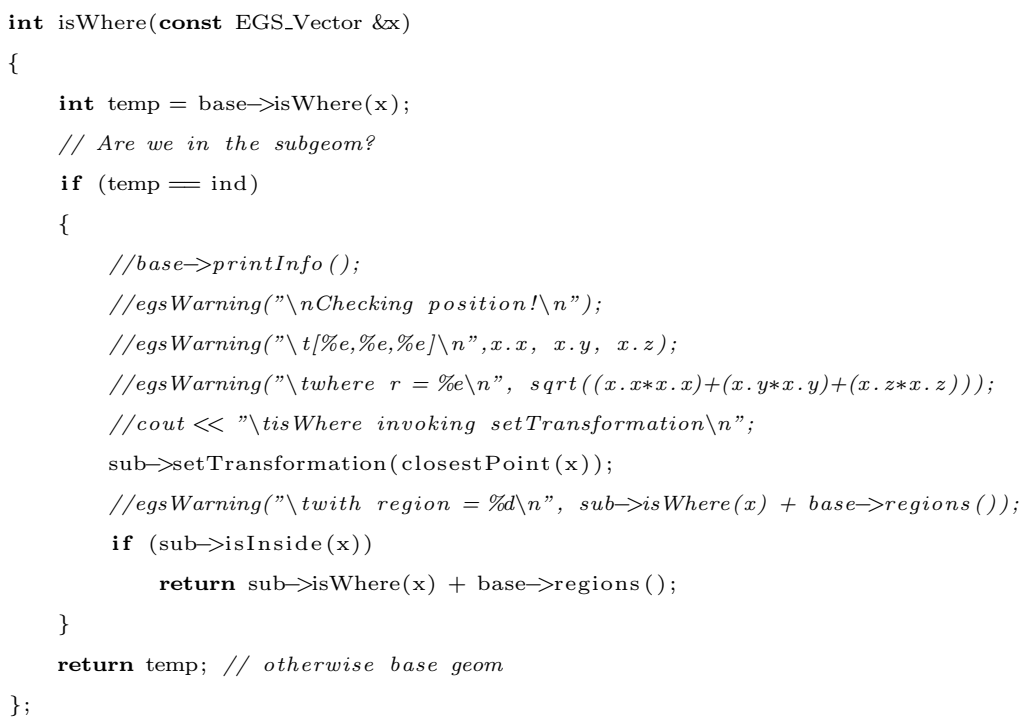




\section{A.3 hownear ()}

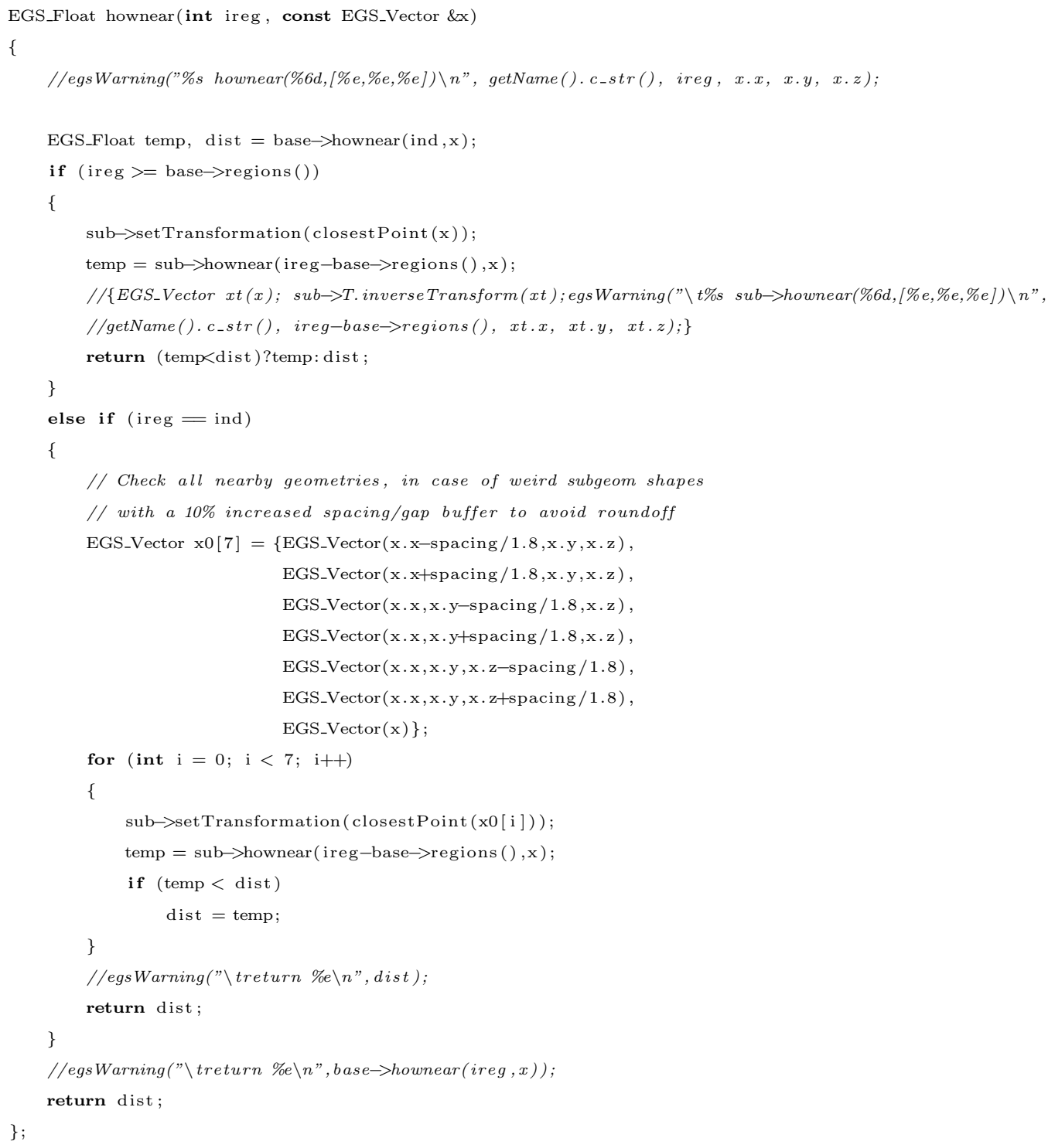




\section{A.4 howfar ()}

int howfar(int ireg, const EGS_Vector \&x, const EGS_Vector \&u, EGS_Float \&t, int *newmed=0, EGS_Vector *normal=0)

\{

//egsWarning("\%s howfar(\%7d,[\%e,\%e,\%e],[\%e,\%e,\%e],\%e)\n",getName().c_str(), ireg, x.x, x.y, x.z, u.x, u.y, u.z, t);

// Catch t=0 exception, which sometimes causes issues when making a lattice of lattices

if $(\mathrm{t}<$ epsilon)

\{

$\mathrm{t}=0.0$

return ireg;

\}

// Are we in the subgeom?

if (ireg $>=$ base $\rightarrow$ regions ())

\{

sub $\rightarrow$ setTransformation ( closestPoint $(x))$;

// Do the howfar call

EGS_Float tempT $=\mathrm{t}$;

$/ /\{$ EGS_Vector $x t(x) ;$ sub $\rightarrow$ T. inverseTransform $(x t) ;$ egsWarning("\%s sub $\rightarrow$ howfar $(\% 7 d,[\% e, \% e, \% e],[\% e, \% e, \% e], \% e) \backslash n "$, $/ /$ getName().c_str(),ireg-base->regions(), xt.x, xt.y, xt.z, u.x, u.y, u.z, tempT); $\}$

int tempReg $=$ sub $\rightarrow$ howfar $($ ireg-base $\rightarrow$ regions ()$, \mathrm{x}, \mathrm{u}$, tempT, newmed, normal $)$;

// If we do leave the subgeom, we want to return the index

// of the region of the base geometry we would be entering,

// which is not necessarily ind if subgeom is at a boundary

EGS_Vector newX $=\mathrm{u}$; newX.normalize ()$; \operatorname{newX}=\mathrm{x}+($ newX $*$ tempT $)$;

if (base->isWhere(newX) != ind) // If we leave ind region of base

\{

$\mathrm{t}=\mathrm{tempT}$

//egsWarning("\tbase->howfar(\%6d, [\%e,\%e,\%e], [\%e,\%e,\%e], \%e)\n",ind, x.x, x.y, x.z, u.x, u.y, u.z, t); tempReg $=$ base $\rightarrow$ howfar $($ ind $, \mathrm{x}, \mathrm{u}, \mathrm{t}, 0$, normal $)$;

if (newmed \&\& tempReg $>=0$ )

*newmed $=$ base $\rightarrow$ medium (tempReg);

//egsWarning("\treturn \%d (\%e)\n",tempReg, $t$ );

//egsWarning("\tnew x should be[\%e,\%e,\%e] $\backslash n$ ", x. $x+t * u . x, x . y+t * u . y, x . z+t * u . z)$;

if $(\mathrm{t}<0)$

\{

egsWarning("Returning_negative_t_from_subgeom_of_\%s $\backslash n$ ", getName ( ) . c_str ( ) );

egsWarning("howfar(\%7d,[\%e,\%e,\%e],[\%e,\%e,\%e],\%e) \n", ireg, x.x, x.y, x.z, u.x, u.y, u.z, t);

//egsFatal("Break 1 !n");

\}

return tempReg;

\}

if $(!($ tempReg+1)) // If we leave sub geom entirely

\{

//egsWarning("\treturn \%d (\%e)\n",tempReg+base->regions(), $t$ );

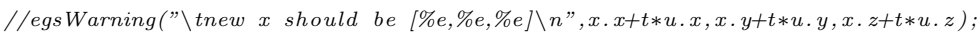

if (newmed) 


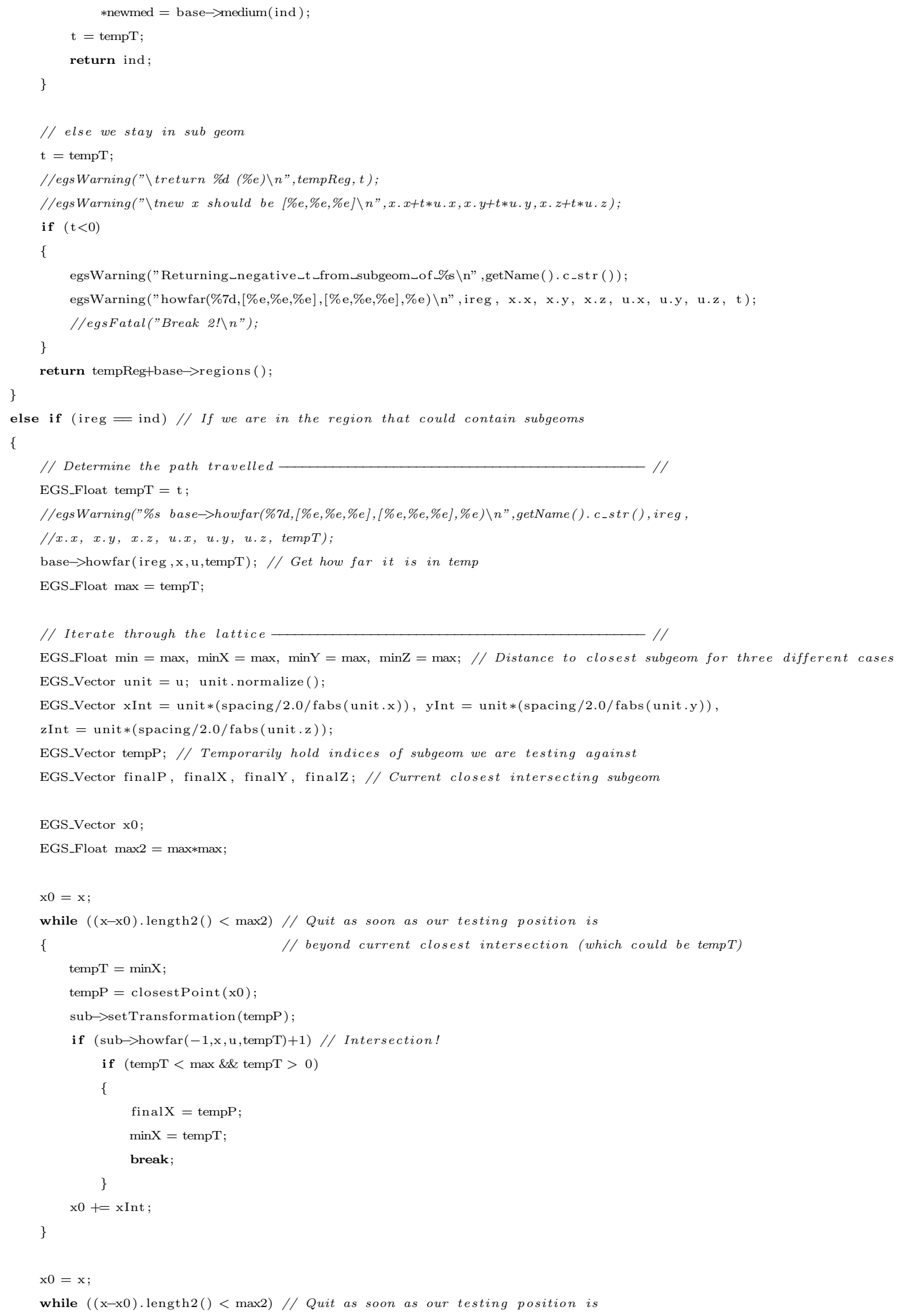


temp $\mathrm{T}=\min Y ;$

tempP $=$ closestPoint $(\mathrm{x} 0) ;$

sub $\rightarrow$ setTransformation (tempP);

if $(\mathrm{sub} \rightarrow \operatorname{howfar}(-1, \mathrm{x}, \mathrm{u}, \mathrm{tempT})+1) / /$ Intersection!

if $($ temp $T<\max \& \&$ temp $T>0)$

\{

finalY $=$ tempP;

$\min Y=$ tempT;

break;

\}

$\mathrm{x} 0+=\mathrm{yInt}$;

\}

$\mathrm{x} 0=\mathrm{x} ;$

while $((\mathrm{x}-\mathrm{x} 0)$. length 2()$<\max 2) / /$ Quit as soon as our testing position is

\{

// beyond current closest intersection (which could be tempT)

tempT $=\operatorname{minZ} ;$

tempP $=$ closestPoint $(\mathrm{x} 0)$;

sub $\rightarrow$ setTransformation (tempP);

if $(\operatorname{sub} \rightarrow \operatorname{howfar}(-1, \mathrm{x}, \mathrm{u}, \operatorname{temp} \mathrm{T})+1) / /$ Intersection!

if $($ temp $\mathrm{T}<\max \& \&$ tempT $>0)$

\{

finalZ $=$ tempP

$\min \mathrm{Z}=\mathrm{tempT}$

break;

\}

$\mathrm{x} 0+=\mathrm{zInt}$;

\}

finalP $=$ final $X ; \min =\min X ;$

if $(\min >\min Y)\{\min =\min Y ;$ finalP $=$ final $Y ;\}$

if $(\min >\min Z)\{\min =\min Z ;$ finalP $=$ final $;\}$

// Check last point

temp $\mathrm{T}=\max ;$

sub $\rightarrow$ setTransformation (closestPoint $(x+$ unit*tempT) );

$\operatorname{sub} \rightarrow \operatorname{howfar}(-1, \mathrm{x}, \mathrm{u}, \operatorname{tempT})$;

if $($ temp $T<\min \& \&$ temp $T>0)$

\{

$\min =$ temp $\mathrm{T}$

finalP $=\operatorname{closestPoint}(x+$ unit $*$ temp $T)$;

\}

$/ /$ We did intersect subgeom

if $(\min <\max )$

\{

tempT $=\mathrm{t} ;$

sub $\rightarrow$ setTransformation (finalP);

int tempReg $=$ sub $\rightarrow$ howfar $(-1, \mathrm{x}, \mathrm{u}$, tempT, newmed, normal $)$;

if $($ tempReg+1)

\{

if (newmed \&\& tempReg $>=0$ )

$*$ newmed $=$ sub $\rightarrow$ medium $($ tempReg $)$;

$\mathrm{t}=\operatorname{tempT}$ 


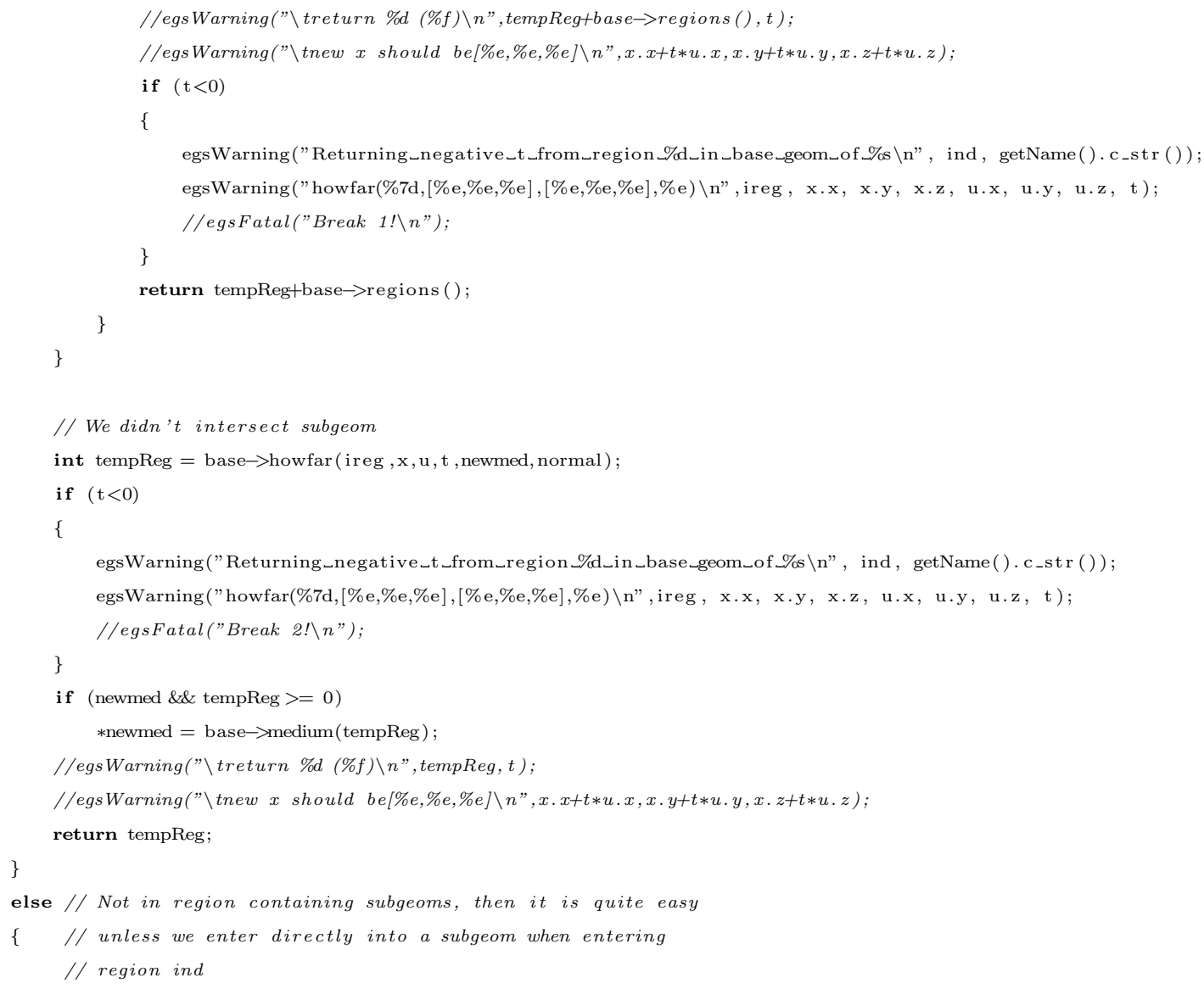


if (newmed \&\& tempReg $>=0$ )

*newmed $=$ base $\rightarrow$ medium (tempReg) ;

//egsWarning("\treturn \%d (\%e)\n",tempReg, $t)$;

//egsWarning("\tnew x should be[\%e,\%e,\%e] $\backslash n ", x . x+t * u . x, x . y+t * u . y, x . z+t * u . z)$;

if $(\mathrm{t}<0)$

\{

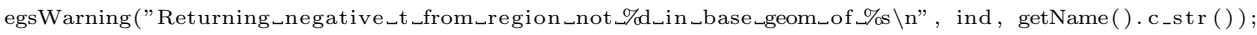

egsWarning("howfar(\%7d,[\%e,\%e,\%e],[\%e,\%e,\%e],\%e)\n",ireg, x.x, x.y, x.z, u.x, u.y, u.z, t);

//egsFatal("Break 2! $n ")$;

\}

return tempReg;

\}

\} 


\section{Appendix B}

\section{Hexagonal lattice implementation}

This section contains the isWhere(), hownear() and howfar() implementations in the egs_hexagonal_lattice c++ geometry created for the thesis as well as the closestPoint() function used throughout them. Here, the variable spacing and gap are defined at construction, where spacing centre-to-centre distance between two neighbouring subgeometries and gap is the spacing between different layers on the z-axis.

Full geometry class available at https://github.com/MartinMartinov/EGSnrc/ at the time of publication, but a more general, clean implementation will be pushed to the main EGSnrc distribution in the future.

\section{B.1 closestPoint()}

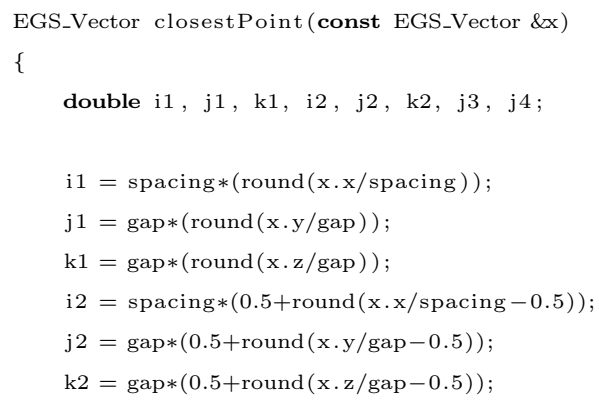


$\mathrm{j} 3=\operatorname{gap} *(-0.25+\operatorname{round}(\mathrm{x} \cdot \mathrm{y} / \mathrm{gap}+0.25)) ;$

$\mathrm{j} 4=\operatorname{gap} *(0.25+\operatorname{round}(\mathrm{x} \cdot \mathrm{y} / \mathrm{gap}-0.25)) ;$

EGS_Vector p1(i1, j1,k1);

EGS_Vector p2(i1, j3 ,k2);

EGS_Vector p3(i2, j2,k1);

EGS_Vector $\mathrm{p} 4(\mathrm{i} 2, \mathrm{j} 4, \mathrm{k} 2)$;

EGS_Float dist $1=(\mathrm{x}-\mathrm{p} 1)$. length2 () ;

EGS_Float dist $2=(\mathrm{x}-\mathrm{p} 2)$. length2 () ;

EGS_Float dist $3=(\mathrm{x}-\mathrm{p} 3)$. length2 () ;

EGS_Float dist $4=(\mathrm{x}-\mathrm{p} 4)$. length2 () ;

if $(\operatorname{dist} 1<\operatorname{dist} 2 \& \&$ dist $1<\operatorname{dist} 3 \& \& \operatorname{dist} 1<\operatorname{dist} 4)$

\{

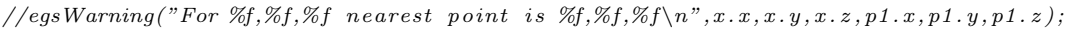

return $\mathrm{p} 1$;

\}

if $(\operatorname{dist} 2<\operatorname{dist} 3 \& \& \operatorname{dist} 2<\operatorname{dist} 4)$

\{

//egsWarning("For \%f,\%f,\%f nearest point is \%f,\%f, \%f\n",x.x,x.y,x.z,p2.x,p2.y,p2.z);

return $\mathrm{p} 2$;

\}

if $(\operatorname{dist} 3<\operatorname{dist} 4)$

\{

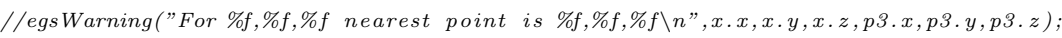
return $\mathrm{p} 3$;

\}

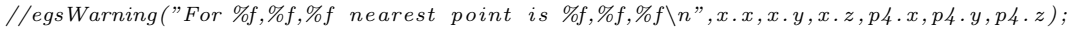

return $\mathrm{p} 4$;

\}; 


\section{B.2 is Where()}

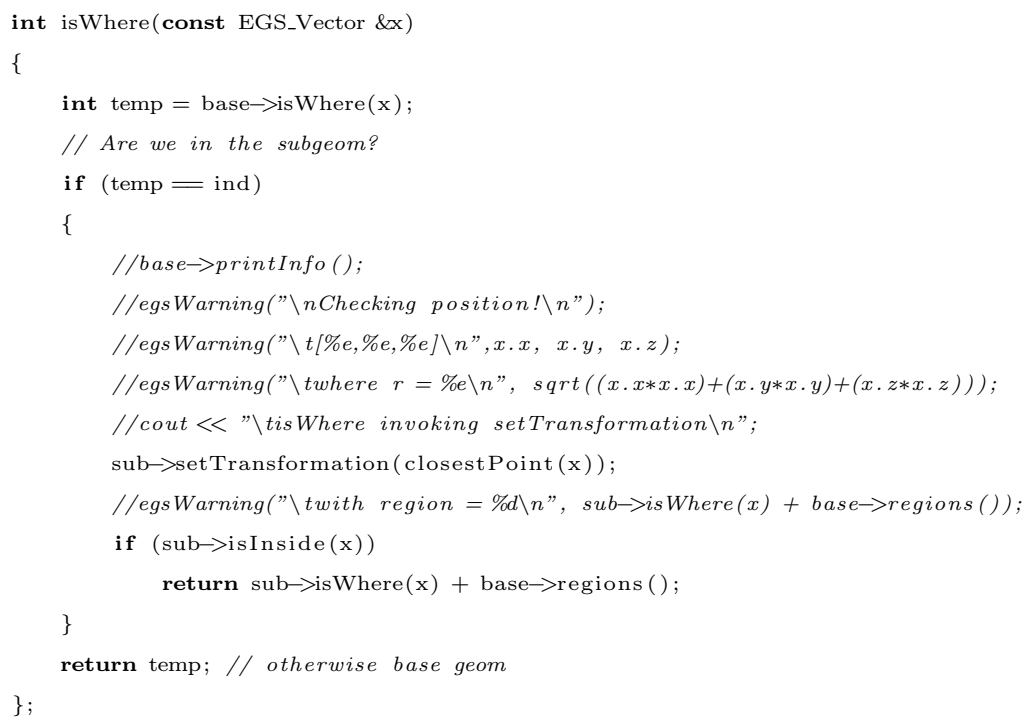




\section{B.3 hownear ()}

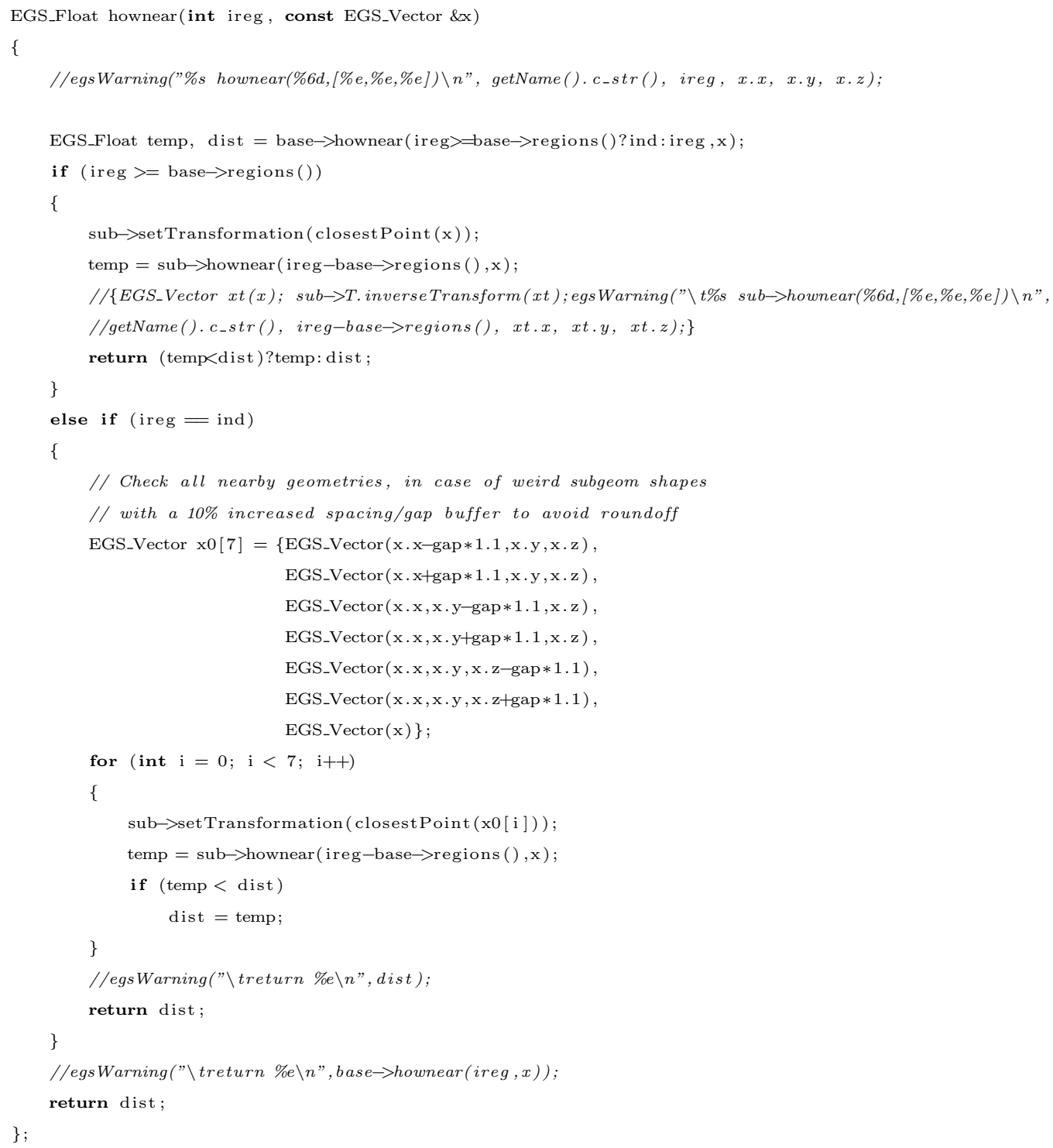




\section{B.4 howfar()}

int howfar(int ireg, const EGS_Vector \&x, const EGS_Vector \&u, EGS_Float \&t, int *newmed=0, EGS_Vector $*$ normal=0)

\{

//egsWarning("\%s howfar(\%7d,[\%e,\%e,\%e],[\%e,\%e,\%e],\%e)\n",getName().c_str(), ireg, x.x, x.y, x.z, u.x, u.y, u.z, t);

// Catch t=0 exception, which sometimes causes issues when making a lattice of lattices

if $(\mathrm{t}<$ epsilon)

\{

$\mathrm{t}=0.0$

return ireg;

\}

// Are we in the subgeom?

if $($ ireg $>=$ base $\rightarrow$ regions ())

\{

sub $\rightarrow$ setTransformation ( closestPoint ( $x)$ );

// Do the howfar call

EGS_Float tempT $=\mathrm{t}$;

$/ /\{$ EGS_Vector $x t(x) ;$ sub $\rightarrow$ T. inverseTransform $(x t) ;$ egsWarning("\%s sub $\rightarrow$ howfar $(\% 7 d,[\% e, \% e, \% e],[\% e, \% e, \% e], \% e) \backslash n "$, $/ / \operatorname{getName}() . c_{-} \operatorname{str}($ ),ireg-base->regions(), xt.x, xt.y, xt.z, u.x, u.y, u.z, tempT); $\}$

int tempReg $=$ sub $\rightarrow$ howfar $($ ireg-base $\rightarrow$ regions ()$, \mathrm{x}, \mathrm{u}$, tempT, newmed, normal $)$;

// If we do leave the subgeom, we want to return the index

// of the region of the base geometry we would be entering,

// which is not necessarily ind if subgeom is at a boundary

EGS_Vector newX $=\mathrm{u}$; newX.normalize ()$; \operatorname{newX}=\mathrm{x}+($ newX $*$ tempT $)$;

if (base->isWhere(newX) != ind) // If we leave ind region of base

\{

$\mathrm{t}=\mathrm{tempT}$

//egsWarning("\tbase->howfar(\%6d, [\%e,\%e,\%e], [\%e,\%e,\%e], \%e)\n",ind, x.x, x.y, x.z, u.x, u.y, u.z, t); tempReg $=$ base $\rightarrow$ howfar $($ ind $, \mathrm{x}, \mathrm{u}, \mathrm{t}, 0$, normal $)$;

if (newmed \&\& tempReg $>=0$ )

*newmed $=$ base $\rightarrow$ medium (tempReg);

//egsWarning("\treturn \%d (\%e)\n",tempReg, $t$ );

//egsWarning("\tnew x should be [\%e,\%e,\%e] $\backslash n ", x . x+t * u . x, x . y+t * u . y, x . z+t * u . z)$;

if $(\mathrm{t}<0)$

\{

egsWarning("Returning_negative_t_from_subgeom_of_\%s $\backslash n$ ", getName ( ) . c_str ( ) );

egsWarning("howfar(\%7d,[\%e,\%e,\%e],[\%e,\%e,\%e],\%e) \n", ireg, x.x, x.y, x.z, u.x, u.y, u.z, t);

//egsFatal("Break 1 !n");

\}

return tempReg;

\}

if $(!($ tempReg+1)) // If we leave sub geom entirely

\{

//egsWarning("\treturn \%d (\%e)\n",tempReg+base->regions(), $t$ );

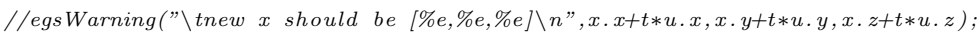

if (newmed) 


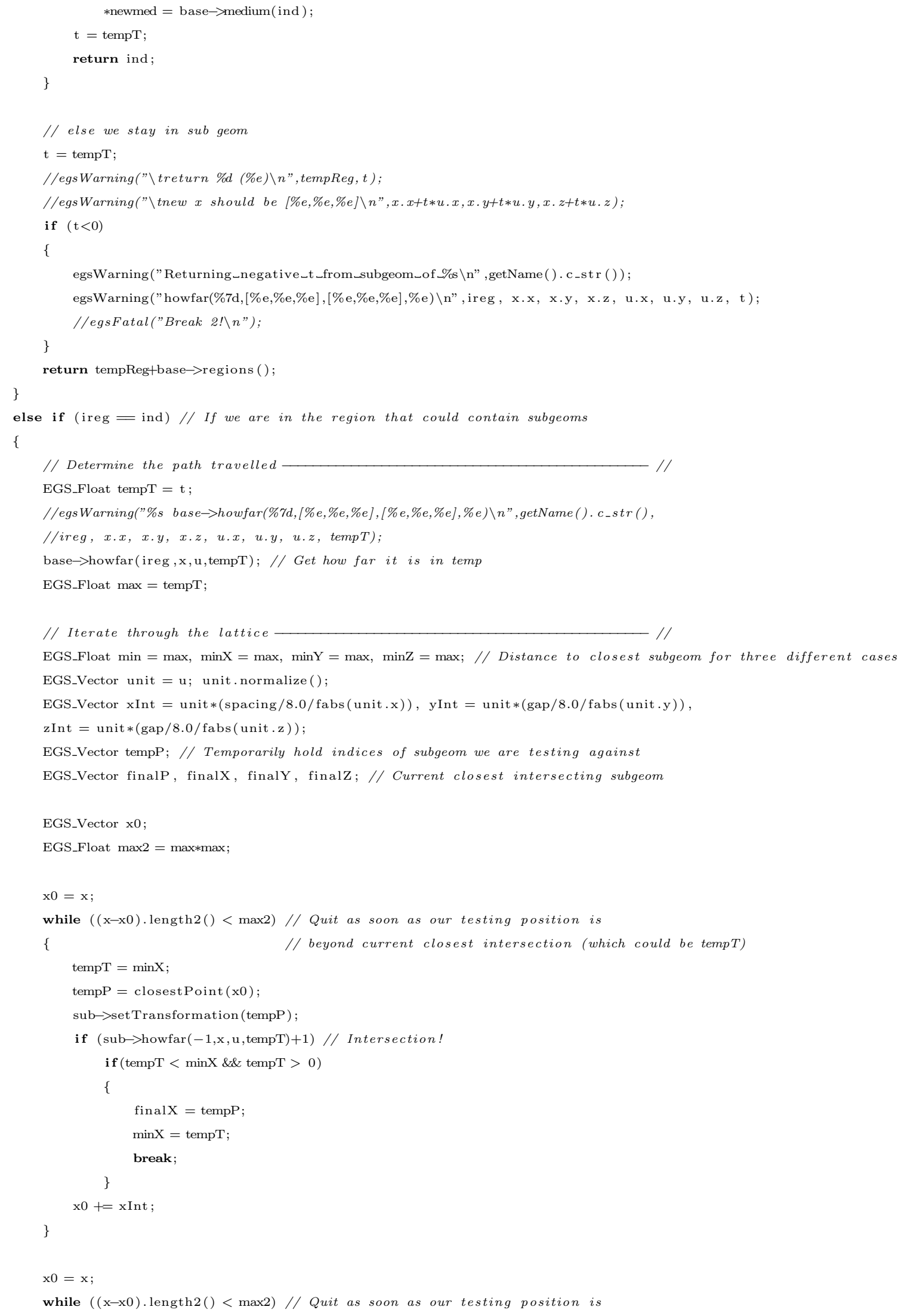




$$
\text { temp } \mathrm{T}=\min Y \text {; }
$$




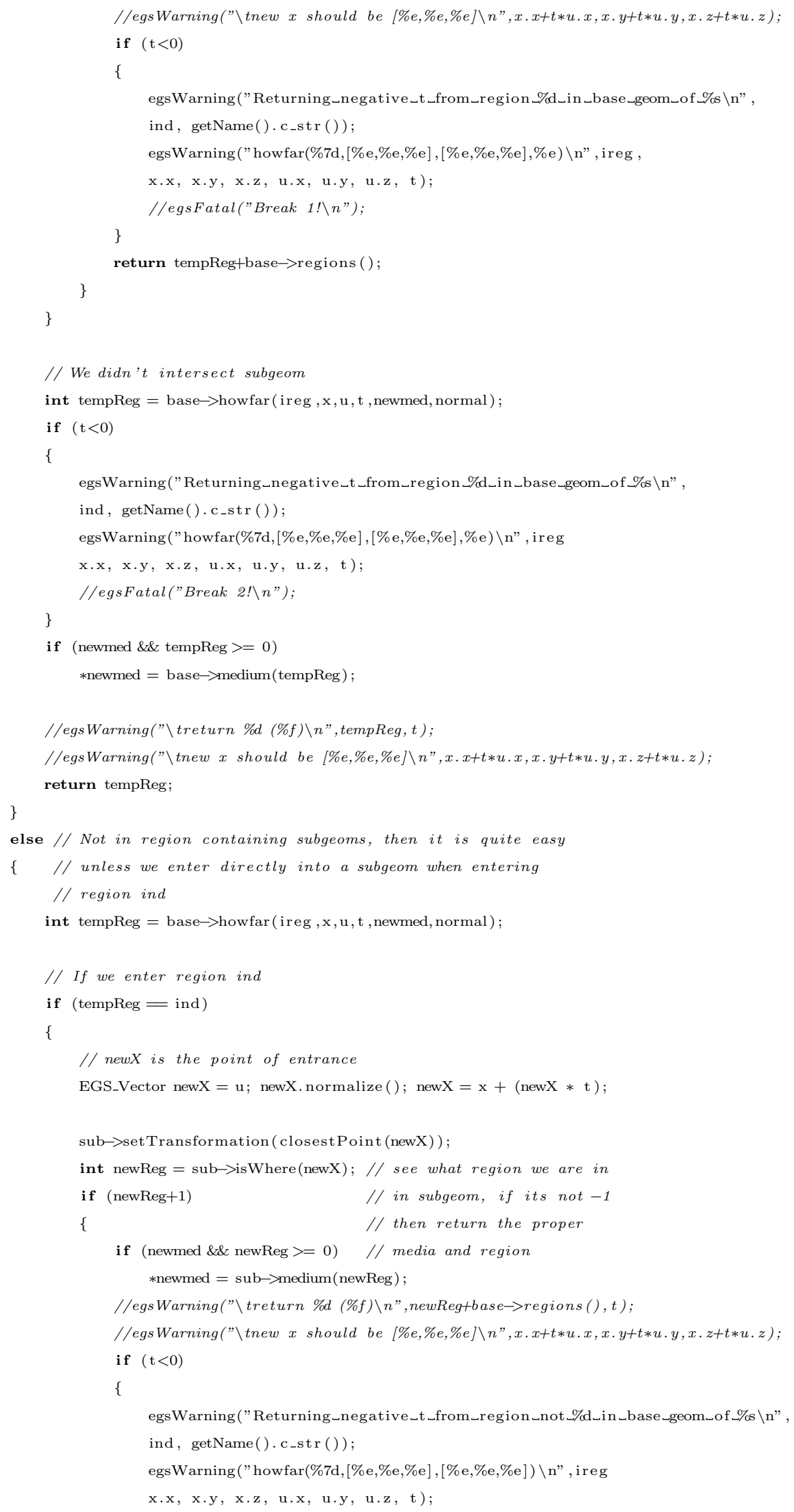




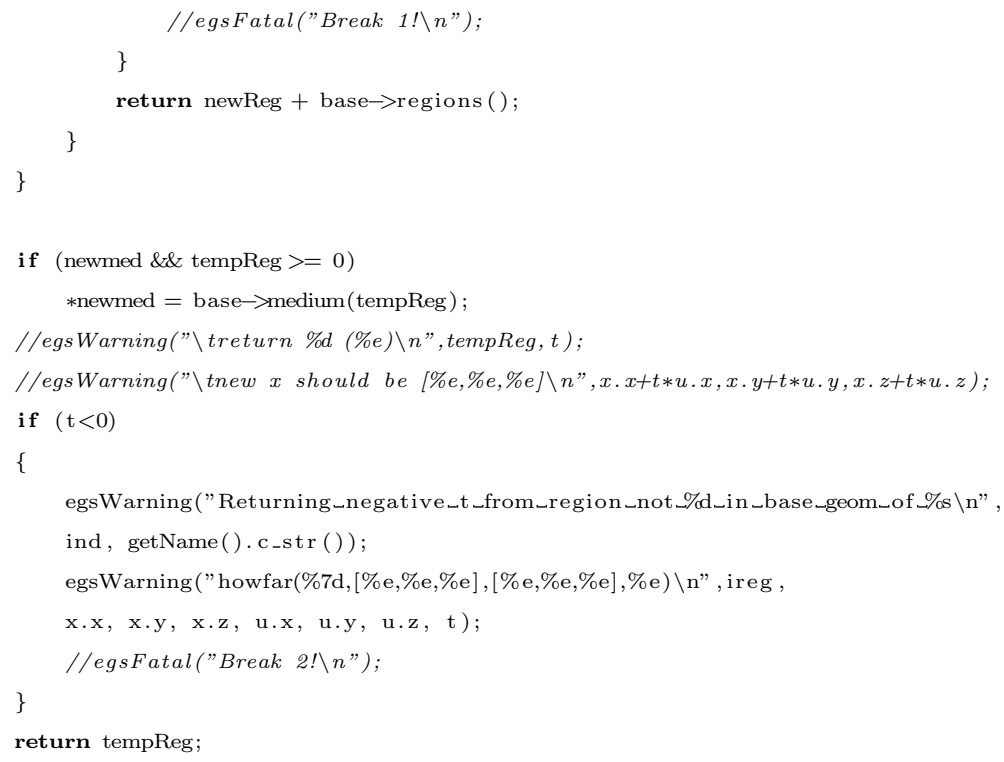




\section{Appendix $\mathrm{C}$}

\section{Homogeneous to cellular DEF}

\section{conversion factors}

This section provides conversion factors to calculate cell nDEF and cDEF using the DEF of a homogeneous gold-tissue mixture in tissue with a gold concentration of 5, 10 and $20 \mathrm{mg} / \mathrm{g}$ for "peri", "4-endo", and "1-endo" (when allowed) gold configurations within the cells at source energies of 20 and $90 \mathrm{keV}$, as well as an ${ }^{125} \mathrm{I}$ spectrum. These data are calculated using the cell DEFs computed in chapter 5 (plotted in figure 5.4) at depths of 0.0126, 0.4914 and $0.9954 \mathrm{~cm}$ and dividing them by the analogous DEF when the microscopic cavities are entirely replaced by tissue (in the no gold case) and homogeneous gold-tissue mixture; the entire mass within the buffer is the scoring volume. This table serves as an example of conversion factors that could be used to determine cell DEF using very simple homogeneous gold-tissue DEF calculations. It must be stressed, as discussed in chapter 5, that these conversion factors should not be used for any GNPT scenarios where the gold concentration varies with position as the relationship between the homogeneous mixture and cell models is far more complex. 
Table C.1: Conversion factors, $\frac{\mathrm{D}_{\text {target }}}{\mathrm{D}_{\text {homog }}}$, with absolute $(k=1)$ uncertainty, $s$, that can be used to calculate cellular (nucleus and cytoplasm) DEFs using the equivalent homogeneous DEFs at 3 different depths (relevant for low energies) in $20 \mathrm{mg} / \mathrm{g}$ gold concentration gold-tissue mixture.

\begin{tabular}{|c|c|c|c|c|c|c|c|c|}
\hline \multirow{2}{*}{ source } & \multirow{2}{*}{ config. } & \multirow{2}{*}{ target } & \multicolumn{2}{|c|}{ surface } & \multicolumn{2}{|c|}{ depth $\approx 0.5 \mathrm{~cm}$} & \multicolumn{2}{|c|}{ depth $\approx 1 \mathrm{~cm}$} \\
\hline & & & $\frac{D_{\text {target }}}{D_{\text {homog }}}$ & $s$ & $\frac{\mathrm{D}_{\text {target }}}{\mathrm{D}_{\text {homog }}}$ & $s$ & $\frac{D_{\text {target }}}{D_{\text {homog }}}$ & $s$ \\
\hline \multirow{4}{*}{$20 \mathrm{keV}$} & \multirow{2}{*}{ peri } & nucleus & 1.6849 & 0.0657 & 1.7205 & 0.0391 & 1.8489 & 0.0255 \\
\hline & & cytoplasm & 0.9818 & 0.0305 & 1.0486 & 0.0194 & 0.6508 & 0.0155 \\
\hline & \multirow{2}{*}{ 4-endo } & nucleus & 0.4475 & 0.0270 & 0.4759 & 0.0175 & 0.5282 & 0.0123 \\
\hline & & cytoplasm & 0.6204 & 0.0244 & 1.0722 & 0.0125 & 0.6942 & 0.0103 \\
\hline \multirow{4}{*}{$90 \mathrm{keV}$} & \multirow{2}{*}{ peri } & nucleus & 1.2870 & 0.0464 & 1.2623 & 0.0459 & 1.2868 & 0.0455 \\
\hline & & cytoplasm & 1.0150 & 0.0310 & 0.9779 & 0.0305 & 1.0077 & 0.0304 \\
\hline & \multirow{2}{*}{ 4-endo } & nucleus & 0.7222 & 0.0321 & 0.7119 & 0.0319 & 0.7247 & 0.0316 \\
\hline & & cytoplasm & 0.8377 & 0.0283 & 0.8013 & 0.0278 & 0.8247 & 0.0276 \\
\hline \multirow{4}{*}{${ }^{125} \mathrm{I}$} & \multirow{2}{*}{ peri } & nucleus & 1.6540 & 0.1068 & 1.6837 & 0.0871 & 1.6618 & 0.0700 \\
\hline & & cytoplasm & 1.0173 & 0.0548 & 1.0501 & 0.0454 & 1.0551 & 0.0374 \\
\hline & \multirow{2}{*}{ 4-endo } & nucleus & 0.5515 & 0.0505 & 0.5718 & 0.0419 & 0.5593 & 0.0344 \\
\hline & & cytoplasm & 0.7252 & 0.0463 & 0.7354 & 0.0379 & 0.7256 & 0.0311 \\
\hline
\end{tabular}


Table C.2: Conversion factors, $\frac{D_{\text {target }}}{D_{\text {homog }}}$, with absolute $(k=1)$ uncertainty, $s$, that can be used to calculate cellular (nucleus and cytoplasm) DEFs using the equivalent homogeneous DEFs at 3 different depths (relevant for low energies) in $10 \mathrm{mg} / \mathrm{g}$ gold concentration gold-tissue mixture.

\begin{tabular}{|c|c|c|c|c|c|c|c|c|}
\hline \multirow{2}{*}{ source } & \multirow{2}{*}{ config. } & \multirow{2}{*}{ target } & \multicolumn{2}{|c|}{ surface } & \multicolumn{2}{|c|}{ depth $\approx 0.5 \mathrm{~cm}$} & \multicolumn{2}{|c|}{ depth $\approx 1 \mathrm{~cm}$} \\
\hline & & & $\frac{D_{\text {target }}}{D_{\text {homog }}}$ & $s$ & $\frac{D_{\text {target }}}{D_{\text {homog }}}$ & $s$ & $\frac{D_{\text {target }}}{D_{\text {homog }}}$ & $s$ \\
\hline \multirow{6}{*}{$20 \mathrm{keV}$} & \multirow{2}{*}{ peri } & nucleus & 1.503 & 0.019 & 1.543 & 0.023 & 1.570 & 0.029 \\
\hline & & cytoplasm & 0.992 & 0.009 & 1.040 & 0.012 & 1.053 & 0.015 \\
\hline & \multirow{2}{*}{ 4-endo } & nucleus & 0.573 & 0.008 & 0.605 & 0.010 & 0.614 & 0.014 \\
\hline & & cytoplasm & 0.759 & 0.007 & 0.781 & 0.009 & 0.784 & 0.012 \\
\hline & \multirow{2}{*}{ 1-endo } & nucleus & 0.552 & 0.008 & 0.579 & 0.010 & 0.593 & 0.013 \\
\hline & & cytoplasm & 0.644 & 0.006 & 0.685 & 0.008 & 0.690 & 0.010 \\
\hline \multirow{6}{*}{$90 \mathrm{keV}$} & \multirow{2}{*}{ peri } & nucleus & 1.228 & 0.018 & 1.187 & 0.018 & 1.209 & 0.019 \\
\hline & & cytoplasm & 1.004 & 0.012 & 0.990 & 0.012 & 0.988 & 0.013 \\
\hline & \multirow{2}{*}{ 4-endo } & nucleus & 0.796 & 0.013 & 0.772 & 0.012 & 0.794 & 0.013 \\
\hline & & cytoplasm & 0.883 & 0.011 & 0.879 & 0.011 & 0.881 & 0.011 \\
\hline & \multirow{2}{*}{ 1-endo } & nucleus & 0.796 & 0.013 & 0.781 & 0.012 & 0.796 & 0.013 \\
\hline & & cytoplasm & 0.842 & 0.010 & 0.842 & 0.010 & 0.840 & 0.011 \\
\hline \multirow{6}{*}{$\mathrm{I}^{125}$} & \multirow{2}{*}{ peri } & nucleus & 1.479 & 0.018 & 1.520 & 0.023 & 1.539 & 0.028 \\
\hline & & cytoplasm & 0.997 & 0.009 & 1.016 & 0.012 & 1.035 & 0.015 \\
\hline & \multirow{2}{*}{ 4-endo } & nucleus & 0.557 & 0.008 & 0.591 & 0.010 & 0.601 & 0.013 \\
\hline & & cytoplasm & 0.757 & 0.007 & 0.766 & 0.009 & 0.765 & 0.011 \\
\hline & \multirow{2}{*}{ 1-endo } & nucleus & 0.535 & 0.008 & 0.576 & 0.010 & 0.575 & 0.013 \\
\hline & & cytoplasm & 0.641 & 0.006 & 0.672 & 0.008 & 0.689 & 0.010 \\
\hline
\end{tabular}


Table C.3: Conversion factors, $\frac{D_{\text {target }}}{D_{\text {homog }}}$, with absolute $(k=1)$ uncertainty, $s$, that can be used to calculate cellular (nucleus and cytoplasm) DEFs using the equivalent homogeneous DEFs at 3 different depths (relevant for low energies) in $5 \mathrm{mg} / \mathrm{g}$ gold concentration gold-tissue mixture.

\begin{tabular}{|c|c|c|c|c|c|c|c|c|}
\hline \multirow{2}{*}{ source } & \multirow{2}{*}{ config. } & \multirow{2}{*}{ target } & \multicolumn{2}{|c|}{ surface } & \multicolumn{2}{|c|}{ depth $\approx 0.5 \mathrm{~cm}$} & \multicolumn{2}{|c|}{ depth $\approx 1 \mathrm{~cm}$} \\
\hline & & & $\frac{D_{\text {target }}}{D_{\text {homog }}}$ & $s$ & $\frac{D_{\text {target }}}{D_{\text {homog }}}$ & $s$ & $\frac{D_{\text {target }}}{D_{\text {homog }}}$ & $s$ \\
\hline \multirow{6}{*}{$20 \mathrm{keV}$} & \multirow{2}{*}{ peri } & nucleus & 1.369 & 0.018 & 1.349 & 0.021 & 1.377 & 0.026 \\
\hline & & cytoplasm & 0.994 & 0.010 & 1.027 & 0.012 & 1.036 & 0.015 \\
\hline & \multirow{2}{*}{ 4-endo } & nucleus & 0.721 & 0.011 & 0.715 & 0.012 & 0.743 & 0.016 \\
\hline & & cytoplasm & 0.874 & 0.009 & 0.877 & 0.010 & 0.870 & 0.013 \\
\hline & \multirow{2}{*}{ 1-endo } & nucleus & 0.715 & 0.010 & 0.713 & 0.012 & 0.702 & 0.015 \\
\hline & & cytoplasm & 0.791 & 0.008 & 0.822 & 0.010 & 0.807 & 0.012 \\
\hline \multirow{6}{*}{$90 \mathrm{keV}$} & \multirow{2}{*}{ peri } & nucleus & 1.170 & 0.016 & 1.138 & 0.016 & 1.136 & 0.017 \\
\hline & & cytoplasm & 1.005 & 0.011 & 0.968 & 0.011 & 0.980 & 0.011 \\
\hline & \multirow{2}{*}{ 4-endo } & nucleus & 0.862 & 0.013 & 0.869 & 0.013 & 0.863 & 0.013 \\
\hline & & cytoplasm & 0.950 & 0.011 & 0.926 & 0.010 & 0.932 & 0.011 \\
\hline & \multirow{2}{*}{ 1-endo } & nucleus & 0.877 & 0.013 & 0.865 & 0.013 & 0.859 & 0.013 \\
\hline & & cytoplasm & 0.922 & 0.010 & 0.892 & 0.010 & 0.892 & 0.011 \\
\hline \multirow{6}{*}{$\mathrm{I}^{125}$} & \multirow{2}{*}{ peri } & nucleus & 1.319 & 0.025 & 1.341 & 0.027 & 1.311 & 0.029 \\
\hline & & cytoplasm & 1.008 & 0.015 & 1.028 & 0.016 & 1.006 & 0.017 \\
\hline & \multirow{2}{*}{ 4-endo } & nucleus & 0.756 & 0.015 & 0.756 & 0.016 & 0.749 & 0.018 \\
\hline & & cytoplasm & 0.938 & 0.014 & 0.934 & 0.015 & 0.912 & 0.016 \\
\hline & \multirow{2}{*}{ 1-endo } & nucleus & 0.732 & 0.015 & 0.733 & 0.016 & 0.719 & 0.017 \\
\hline & & cytoplasm & 0.829 & 0.013 & 0.844 & 0.014 & 0.822 & 0.014 \\
\hline
\end{tabular}




\section{References}

1 H. E. Johns, L. M. Bates, E. R. Epp, D. V. Cormack, S. O. Fedoruk, A. Morrison, W. R. Dixon, and C. Garrett, 1000-Curie Cobalt-60 Units for Radiation Therapy, Nature 168, 1035 - 1036 (1951).

2 H. Johns, L. Bates, and T. Watson, 1000 Curie cobalt units for radiation therapy. I. The Saskatchewan cobalt 60 unit., Brit. J. Radiol. 25, 296 (1952).

3 H. Johns, D. Cormack, and S. Fedoruk, II. Depth dose data and diaphragm design for the Saskatchewan 1000 Curie cobalt unit, Brit. J. Radiol. 25, 302-308 (1952).

4 H. E. Johns, The physicist in cancer treatment and detection, Int. J. Radiat. Oncol. Biol. Phys. 7, 801-808 (1981).

5 H. E. Johns and J. R. Cunningham, The physics of radiology, 4th ed., Charles C. Thomas, Springfield, Illinois, 1983.

6 E. B. Podgorsak, editor, Radiation Oncology Physics: A Handbook for Teachers and Students, Educational Report Series, IAEA, Vienna, Austria, 2005.

7 M. Lodge, M. Pijls-Johannesma, L. Stirk, A. J. Munro, D. De Ruysscher, and T. Jefferson, A systematic literature review of the clinical and cost-effectiveness of hadron therapy in cancer, Radiother. Oncol. 83, 110-122 (2007).

8 M. J. Rivard, B. M. Coursey, L. A. DeWerd, M. S. Huq, G. S. Ibbott, M. G. Mitch, R. Nath, and J. F. Williamson, Update of AAPM Task Group No. 43 Report: A revised AAPM protocol for brachytherapy dose calculations, Med. Phys. 31, 633 - 674 (2004).

9 T. P. Mate, J. E. Gottesman, J. Hatton, M. Gribble, and L. Van Hollebeke, High doserate afterloading 192Iridium prostate brachytherapy: feasibility report, Int. J. Radiat. Oncol. Biol. Phys. 41, 525-533 (1998).

10 H. Cember, Introduction to health physics, Number 614.876 CEM, 1996. 
11 P. Riley, Free radicals in biology: oxidative stress and the effects of ionizing radiation, Int. J. Radiat. Biol. 65, 27-33 (1994).

12 O. Desouky, N. Ding, and G. Zhou, Targeted and non-targeted effects of ionizing radiation, Journal of Radiation Research and Applied Sciences 8, 247-254 (2015).

13 B. D. Kavanagh, R. Timmerman, S. H. Benedict, Q. Wu, T. Schefter, K. Stuhr, S. McCourt, F. Newman, R. Cardinale, and L. Gaspar, How should we describe the radiobiologic effect of extracranial stereotactic radiosurgery: Equivalent uniform dose or tumor control probability?, Med. Phys. 30, 321-324 (2003).

S. F. Kry, M. Salehpour, D. S. Followill, M. Stovall, D. A. Kuban, R. A. White, and I. I. Rosen, The calculated risk of fatal secondary malignancies from intensity-modulated radiation therapy, Int. J. Radiat. Oncol. Biol. Phys. 62, 1195-1203 (2005).

S. Jain, D. Hirst, and J. O'sullivan, Gold nanoparticles as novel agents for cancer therapy, Brit. J. Radiol. 85, 101-113 (2012).

16 P. Zygmanski and E. Sajo, Nanoscale radiation transport and clinical beam modeling for Gold Nanoparticle Dose Enhanced Radiotherapy (GNPT) using x-rays, Brit. J. Radiol. , 20150200 (2016).

17 E. B. Podgorsak, editor, Review of Radiation Oncology Physics: A Handbook for Teachers and Students, Educational Report Series, IAEA, Vienna, Austria, 2004.

18 M. J. Berger, J. H. Hubbell, S. M. Seltzer, J. Chang, J. S. Coursey, R. Sukumar, D. S. Zucker, and K. Olsen, XCOM: Photon cross section database (version 1.5), Technical report, NIST, Gaithersburg, MD, http://physics.nist.gov/xcom, 2010.

19 E. B. Podgorsak, Radiation Physics for Medical Physicists, Springer-Verlag, Berlin, Heidelberg, New York, 2005.

ICRU, Tissue Substitutes in Radiation Dosimetry and Measurements, ICRU Report 44, ICRU, Washington D.C., 1989.

21 J. F. Hainfeld, D. N. Slatkin, and H. M. Smilowitz, The use of gold nanoparticles to enhance radiotherapy in mice, Phys. Med. Biol. 49, N309-N315 (2004).

22 S. Jain et al., Cell-specific radiosensitization by gold nanoparticles at megavoltage radiation energies, Int. J. Radiat. Oncol. Biol. Phys. 79, 531-539 (2011).

23 D. Brivio, P. Nguyen, E. Sajo, W. Ngwa, and P. Zygmanski, A Monte Carlo study of I-125 prostate brachytherapy with gold nanoparticles: dose enhancement with simultaneous rectal dose sparing via radiation shielding, Phys. Med. Biol. 62, 1935 (2017). 
S. Asadi, M. Vaez-zadeh, M. Vahidian, M. Marghchouei, and S. F. Masoudi, Ocular brachytherapy dosimetry for 103Pd and 125I in the presence of gold nanoparticles: a Monte Carlo study, J of App. Clin. Med. Phys. 17, 90-99 (2016).

L. Strigari, V. Ferrero, G. Visonà, F. Dalmasso, A. Gobbato, P. Cerello, S. Visentin, and A. Attili, Targeted dose enhancement in radiotherapy for breast cancer using gold nanoparticles, part 2: A treatment planning study, Med. Phys. 44, 1993-2001 (2017).

F. Boateng and W. Ngwa, Modeling gold nanoparticle-eluting spacer degradation during brachytherapy application with in situ dose painting, Brit. J. Radiol. 90, 20170069 (2017).

S. Her, D. A. Jaffray, and C. Allen, Gold nanoparticles for applications in cancer radiotherapy: Mechanisms and recent advancements, Advanced drug delivery reviews 109, 84-101 (2017).

J. Kimling, M. Maier, B. Okenve, V. Kotaidis, H. Ballot, and A. Plech, Turkevich method for gold nanoparticle synthesis revisited, J. of Phys. Chem. B 110, 1570015707 (2006).

M. Treguer-Delapierre, J. Majimel, S. Mornet, E. Duguet, and S. Ravaine, Synthesis of non-spherical gold nanoparticles, Gold bulletin 41, 195-207 (2008).

S. E. Skrabalak, J. Chen, L. Au, X. Lu, X. Li, and Y. Xia, Gold nanocages for biomedical applications, Advanced Materials 19, 3177-3184 (2007).

W. P. Wuelfing, S. M. Gross, D. T. Miles, and R. W. Murray, Nanometer gold clusters protected by surface-bound monolayers of thiolated poly (ethylene glycol) polymer electrolyte, Journal of the American Chemical Society 120, 12696-12697 (1998).

J. M. Bergen, H. A. Von Recum, T. T. Goodman, A. P. Massey, and S. H. Pun, Gold nanoparticles as a versatile platform for optimizing physicochemical parameters for targeted drug delivery, Macromolecular Bioscience 6, 506-516 (2006).

33 N. Sinha, G. Cifter, E. Sajo, R. Kumar, S. Sridhar, P. L. Nguyen, R. A. Cormack, G. M. Makrigiorgos, and W. Ngwa, Brachytherapy Application With In Situ Dose Painting Administered by Gold Nanoparticle Eluters, Int. J. Radiat. Oncol. Biol. Phys. 91, 385-392 (2015).

34 E. E. Connor, J. Mwamuka, A. Gole, C. J. Murphy, and M. D. Wyatt, Gold nanoparticles are taken up by human cells but do not cause acute cytotoxicity, Small 1, 325-327 (2005).

Y.-S. Chen, Y.-C. Hung, I. Liau, and G. S. Huang, Assessment of the in vivo toxicity of gold nanoparticles, Nanoscale research letters 4, 858 (2009). 
M. Arnida, A. Ray, C. Peterson, and H. Ghandehari, Geometry and surface characteristics of gold nanoparticles influence their biodistribution and uptake by macrophages, Eu. J. of Pharma. and Biopharma. 77, 417 (2011).

H. S. Choi, W. Liu, P. Misra, E. Tanaka, J. P. Zimmer, B. I. Ipe, M. G. Bawendi, and J. V. Frangioni, Renal clearance of quantum dots, Nature biotechnology 25, 1165 (2007).

J. Schuemann, R. Berbeco, D. B. Chithrani, S. H. Cho, R. Kumar, S. J. McMahon, S. Sridhar, and S. Krishnan, Roadmap to clinical use of gold nanoparticles for radiation sensitization, Int. J. Radiat. Oncol. Biol. Phys. 94, 189-205 (2016).

D. B. Chithrani, Intracellular uptake, transport, and processing of gold nanostructures, Molecular membrane biology 27, 299-311 (2010).

S. Jain et al., Gold nanoparticle cellular uptake, toxicity and radiosensitisation in hypoxic conditions, Radiother. Oncol. 110, 342-347 (2014).

C.-J. Liu et al., Enhanced x-ray irradiation-induced cancer cell damage by gold nanoparticles treated by a new synthesis method of polyethylene glycol modification, Nanotechnology 19, 295104 (2008).

H. Nikjoo, S. Uehara, and D. Emfietzoglou, Interaction of radiation with matter, CRC press, 2016.

A. F. Bielajew, Fundamentals of the Monte Carlo method for neutral and charged particle transport, The University of Michigan (2001).

I. Kawrakow and D. W. O. Rogers, The EGSnrc Code System: Monte Carlo simulation of electron and photon transport, NRC Technical Report PIRS-701 v4-2-2-5, National Research Council Canada, Ottawa, Canada. http://www.irs.inms.nrc.ca/inms/irs/EGSnrc/EGSnrc.html, 2007.

G. Z. Molière, Theorie der Streuung schneller geladener Teilchen. I. Einzelstreuung am abgeschirmten Coulomb-Field, Z. Naturforsch. A 2, 133 - 145 (1947).

I. Kawrakow, Accurate condensed history Monte Carlo simulation of electron transport. I. EGSnrc, the new EGS4 version, Med. Phys. 27, 485 - 498 (2000).

J. Wulff, K. Zink, and I. Kawrakow, Efficiency improvements for ion chamber calculations in high energy photon beams, Med. Phys. 35, 1328- 1336 (2008).

I. Kawrakow, E. Mainegra-Hing, D. W. O. Rogers, F. Tessier, and B. R. B. Walters, The EGSnrc Code System: Monte Carlo simulation of electron and photon transport, NRC Technical Report PIRS-701 v4-2-32, National Research Council Canada, Ottawa, Canada. http://www.nrccnrc.gc.ca/eng/solutions/advisory/egsnrc/download_egsnrc.html, 2011. 
S. H. Cho, Estimation of tumour dose enhancement due to gold nanoparticles during typical radiation treatments: a preliminary Monte Carlo study, Phys. Med. Biol. 50, N163 (2005).

J. Robar, Generation and modelling of megavoltage photon beams for contrastenhanced radiation therapy, Phys. Med. Biol. 51, 5487 (2006).

M. R. McEwen, I. Kawrakow, and C. K. Ross, The effective point of measurement of ionization chambers and the build-up anomaly in MV x-ray beams, Med. Phys. 35, 950 - 958 (2008).

S. H. Cho, B. L. Jones, and S. Krishnan, The dosimetric feasibility of gold nanoparticleaided radiation therapy (GNRT) via brachytherapy using low-energy gamma-/x-ray sources, Phys. Med. Biol. 54, 4889-4905 (2009).

B. Koger and C. Kirkby, A method for converting dose-to-medium to dose-to-tissue in Monte Carlo studies of gold nanoparticle-enhanced radiotherapy, Phys. Med. Biol. 61, 2014-2024 (2016).

4 B. L. Jones, S. Krishnan, and S. H. Cho, Estimation of microscopic dose enhancement factor around gold nanoparticles by Monte Carlo calculations, Med. Phys. 37, 3809 3816 (2010).

M. K. Leung, J. C. Chow, B. D. Chithrani, M. J. Lee, B. Oms, and D. A. Jaffray, Irradiation of gold nanoparticles by x-rays: Monte Carlo simulation of dose enhancements and the spatial properties of the secondary electrons production, Med. Phys. 38, 624-631 (2011).

6 S. J. McMahon et al., Nanodosimetric effects of gold nanoparticles in megavoltage radiation therapy, Radiother. Oncol. 100, 412-416 (2011).

E. Lechtman, N. Chattopadhyay, Z. Cai, S. Mashouf, R. Reilly, and J. P. Pignol, Implications on clinical scenario of gold nanoparticle radiosensitization in regards to photon energy, nanoparticle size, concentration and location, Phys. Med. Biol. 56, 4631 - 4647 (2011).

M. Douglass, E. Bezak, and S. Penfold, Monte Carlo investigation of the increased radiation deposition due to gold nanoparticles using kilovoltage and megavoltage photons in a 3D randomized cell model, Med. Phys. 40, 071710 (9 pp) (2013).

A. McNamara, W. Kam, N. Scales, S. McMahon, J. Bennett, H. Byrne, J. Schuemann, H. Paganetti, R. Banati, and Z. Kuncic, Dose enhancement effects to the nucleus and mitochondria from gold nanoparticles in the cytosol, Phys. Med. Biol. 61, 5993-6010 (2016). 
60 W. Sung, S.-J. Ye, A. L. McNamara, S. J. McMahon, J. Hainfeld, J. Shin, H. M. Smilowitz, H. Paganetti, and J. Schuemann, Dependence of gold nanoparticle radiosensitization on cell geometry, Nanoscale 9, 5843-5853 (2017).

61 Z. Cai, J. Pignol, N. Chattopadhyay, Y. L. Kwon, E. Lechtman, and R. M. Reilly, Investigation of the effects of cell model and subcellular location of gold nanoparticles on nuclear dose enhancement factors using Monte Carlo simulation, Med. Phys. 40, 114101 (2013).

62 C. Kirkby and E. Ghasroddashti, Targeting mitochondria in cancer cells using gold nanoparticle-enhanced radiotherapy: A Monte Carlo study, Med. Phys. 42, 1119 1128 (2015).

63 S. Asadi, M. Vaez-zadeh, S. F. Masoudi, F. Rahmani, C. Knaup, and A. S. Meigooni, Gold nanoparticle-based brachytherapy enhancement in choroidal melanoma using a full Monte Carlo model of the human eye, J of App. Clin. Med. Phys. 16, 344-357 (2015).

64 W. Ngwa, G. M. Makrigioros, and R. I. Berbeco, Applying gold nanoparticles as tumor-vascular disrupting agents during brachytherapy: estimation of endothelial dose enhancment, Phys. Med. Biol. 55, 6533 - 6548 (2010).

65 W. Ngwa, G. M. Makrigiorgos, and R. I. Berbeco, Gold nanoparticle-aided brachytherapy with vascular dose painting: Estimation of dose enhancement to the tumor endothelial cell nucleus, Med. Phys. 39, 392-398 (2012).

66 W. Ngwa, G. M. Makrigioros, and R. I. Berbeco, Gold nanoparticle enhancement of stereotactic radiosurgery for neovascular age-related macular degeneration, Phys. Med. Biol. 57, $6371-6380$ (2012).

67 G. Cifter, J. Chin, F. Cifter, Y. Altundal, N. Sinha, E. Sajo, and W. Ngwa, Targeted radiotherapy enhancement during electronic brachytherapy of accelerated partial breast irradiation (APBI) using controlled release of gold nanoparticles, Physica Medica 31, 1070-1074 (2015).

68 J. Wulff, J. T. Heverhagen, I. Kawrakow, and K. Zink, Investigation of systematic uncertainties in Monte Carlo calculated beam quality correction factors, in Second European Workshop on Monte Carlo Treatment Planning Programme and Book of Abstracts, page 126, 2009.

69 I. Kawrakow, egspp: the EGSnrc C++ class library, Technical Report PIRS-899, National Research Council Canada, Ottawa, Canada, 2005.

70 M. Šefl, S. Incerti, G. Papamichael, and D. Emfietzoglou, Calculation of cellular Svalues using Geant4-DNA: the effect of cell geometry, Appl. Radiat. Is. 104, 113-123 (2015). 
71 M. I. Aroyo, U. Müller, and H. Wondratschek, Historical introduction, International Tables for Crystallography 1, 2-6 (2006).

72 H. Bouchard, J. de Pooter, A. Bielajew, and S. Duane, Reference dosimetry in the presence of magnetic fields: conditions to validate Monte Carlo simulations, Phys. Med. Biol. 60, 6639 - 6654 (2015).

73 U. Fano, Note on the Bragg-Gray cavity principle for measuring energy dissipation, Radiat. Res. 1, $237-240$ (1954).

74 I. Kawrakow, Accurate condensed history Monte Carlo simulation of electron transport. II. Application to ion chamber response simulations, Med. Phys. 27, 499 - 513 (2000).

75 J. P. Seuntjens, I. Kawrakow, J. Borg, F. Hobeila, and D. W. O. Rogers, Calculated and measured air-kerma response of ionization chambers in low and medium energy photon beams, in Recent developments in accurate radiation dosimetry, Proc. of an Int'l Workshop, edited by J. P. Seuntjens and P. Mobit, pages 69 - 84, Medical Physics Publishing, Madison WI, 2002.

76 P. G. Watson and J. Seuntjens, Technical Note: Effect of explicit M and N-shell atomic transitions on a low-energy x-ray source, Med. Phys. 43, 1760-1763 (2016).

77 A. Mesbahi, F. Jamali, and N. Gharehaghaji, Effect of photon beam energy, gold nanoparticle size and concentration on the dose enhancement in radiation therapy, Bioimpacts 3, 29 (2013).

78 M. Ghorbani, D. Pakravan, M. Bakhshabadi, and A. S. Meigooni, Dose enhancement in brachytherapy in the presence of gold nanoparticles: a Monte Carlo study on the size of gold nanoparticles and method of modelling, Nukleonika 57, 401-406 (2012).

79 M. Ghorbani, M. Bakhshabadi, A. Golshan, and C. Knaup, Dose enhancement by various nanoparticles in prostate brachytherapy, Australasian Physical \& Engineering Sciences in Medicine 36, 431-440 (2013).

80 S. X. Zhang, J. Gao, T. A. Buchholz, Z. Wang, M. R. Salehpour, R. A. Drezek, and T.-K. $\mathrm{Yu}$, Quantifying tumor-selective radiation dose enhancements using gold nanoparticles: a monte carlo simulation study, Biomedical microdevices 11, 925-933 (2009).

81 M. T. B. Toossi, M. Ghorbani, M. Mehrpouyan, F. Akbari, L. S. Sabet, and A. S. Meigooni, A Monte Carlo study on tissue dose enhancement in brachytherapy: a comparison between gadolinium and gold nanoparticles, Australasian Physical \& Engineering Sciences in Medicine 35, 177-185 (2012).

82 R. M. Thomson, Å. Carlsson Tedgren, and J. F. Williamson, On the biological basis for competing macroscopic dose descriptors for kilovoltage dosimetry: cellular dosimetry for brachytherapy and diagnostic radiology, Phys. Med. Biol. 58, 1123 - 1150 (2013). 
J. Sempau and P. Andreo, Configuration of the electron transport algorithm of PENELOPE to simulate ion chambers, Phys. Med. Biol. 51, 3533 - 3548 (2006).

M. J. Berger, ESTAR, PSTAR and ASTAR: Computer Programs for Calculating Stopping-Power and Ranges for Electrons, Protons, and Helium Ions (version 2.0.1), NIST Report NISTIR-4999 (Washington DC), available on-line at http://physics.nist.gov/Star (2017).

E. Poon, J. S. Seuntjens, and F. Verhaegen, Consistency test of the electron transport algorithm in the GEANT4 Monte Carlo code, Phys. Med. Biol. 50, 681 - 694 (2005).

H. Nikjoo, S. Uehara, D. Emfietzoglou, and F. A. Cucinotta, Track-structure codes in radiation research, Radiat. Meas. 41, 1052 - 1074 (2006).

R. M. Thomson and I. Kawrakow, On the Monte Carlo simulation of electron transport in the sub-1 keV energy range, Med. Phys. 38, 4531 - 4534 (2011).

P. Zygmanski, B. Liu, P. Tsiamas, F. Cifter, M. Petersheim, J. Hesser, and E. Sajo, Dependence of Monte Carlo microdosimetric computations on the simulation geometry of gold nanoparticles, Phys. Med. Biol. 58, 7961-7977 (2013).

Y. Lin, S. J. McMahon, M. Scarpelli, H. Paganetti, and J. Schuemann, Comparing gold nano-particle enhanced radiotherapy with protons, megavoltage photons and kilovoltage photons: a Monte Carlo simulation, Phys. Med. Biol. 59, 7675-7689 (2014).

M. J. Berger et al., Stopping-power and range tables for electrons, protons, and helium ions, NIST Physics Laboratory Gaithersburg, MD, 1998.

D. E. Cullen, J. H. Hubbell, and L. Kissel, EDPL97: the Evaluated Photon Data Library, '97 Version, Report UCRL-50400, Vol 6, Rev 5, LLNL, Livermore, Ca, 1997.

J. Lee, J. Lee, D. Ryu, H. Lee, and S.-J. Ye, Fano cavity test for electron Monte Carlo transport algorithms in magnetic fields: comparison between EGSnrc, PENELOPE, MCNP6 and Geant4, Phys. Med. Biol. 63, 195013 (2018).

D. W. O. Rogers and I. Kawrakow, Monte Carlo calculated correction factors for primary standards of air-kerma, Med. Phys. 30, 521 - 543 (2003).

4 J. P. Archambault and E. Mainegra-Hing, Comparison between EGSnrc, Geant4, MCNP5 and Penelope for mono-energetic electron beams, Phys. Med. Biol. 60, 4951 4962 (2015).

J. Crank, The mathematics of diffusion, Oxford university press, 1979.

M. Chamberland, R. Taylor, D. Rogers, and R. Thomson, egs_brachy: a versatile and fast Monte Carlo code for brachytherapy, Phys. Med. Biol. 61, 8214 (2016). 
97

98

J. Dolan, Z. Li, and J. F. Williamson, Monte Carlo and experimental dosimetry of an ${ }^{125}$ I brachytherapy seed, Med. Phys. 33, 4675 - 4684 (2006).

NCRP Report 58, A Handbook of Radioactivity Measurements Procedures, NCRP Publications, 7910 Woodmont Avenue, Bethesda, MD. 20814 USA (1985).

M. K. Murphy, R. K. Piper, L. R. Greenwood, M. G. Mitch, P. J. Lamperti, S. M. Seltzer, M. J. Bales, and M. H. Phillips, Evaluation of the new cesium-131 seed for use in low-energy x-ray brachytherapy, Med. Phys. 31, 1529 - 1538 (2004).

Brookhaven National Laboratory, National Nuclear Data Center, http://www.nndc.bnl.gov/nudat2.

J. I. Monroe and J. F. Williamson, Monte Carlo-aided dosimetry of the Theragenics TheraSeed Model $200{ }^{103} \mathrm{Pd}$ interstitial brachytherapy seed, Med. Phys. 29, $609-621$ (2002).

02 J. C. Roeske, L. Nuñez, M. Hoggarth, E. Labay, and R. R. Weichselbaum, Characterization of the theorectical radiation dose enhancement from nanoparticles, Technology in cancer research \& treatment 6, 395-401 (2007).

03 J. L. Robar, S. A. Riccio, and M. Martin, Tumour dose enhancement using modified megavoltage photon beams and contrast media, Phys. Med. Biol. 47, 2433 (2002).

R. I. Berbeco, W. Ngwa, and G. M. Makrigiorgos, Localized dose enhancement to tumor blood vessel endothelial cells via megavoltage X-rays and targeted gold nanoparticles: new potential for external beam radiotherapy, Int. J. Radiat. Oncol. Biol. Phys. 81, 270-276 (2011).

P. Oliver and R. M. Thomson, A Monte Carlo study of macroscopic and microscopic dose descriptors for kilovoltage cellular dosimetry, Phys. Med. Biol. 62, 1417 (2017).

F. S. Rasouli and S. F. Masoudi, Monte Carlo investigation of the effect of gold nanoparticles' distribution on cellular dose enhancement, Radiat. Phys. Chem. 158, 6-12 (2019).

07 E. Lechtman and J.-P. Pignol, Interplay between the gold nanoparticle sub-cellular localization, size, and the photon energy for radiosensitization, Scientific reports 7, 13268 (2017).

08 D. B. Chithrani, M. Dunne, J. Stewart, C. Allen, and D. A. Jaffray, Cellular uptake and transport of gold nanoparticles incorporated in a liposomal carrier, Nanomedicine: Nanotechnology, Biology and Medicine 6, 161-169 (2010).

B. D. Chithrani and W. C. Chan, Elucidating the mechanism of cellular uptake and removal of protein-coated gold nanoparticles of different sizes and shapes, Nano letters 7, 1542-1550 (2007). 
B. Koger and C. Kirkby, Dosimetric effects of polyethylene glycol surface coatings on gold nanoparticle radiosensitization, Phys. Med. Biol. 62, 8455 (2017).

C. Kirkby, B. Koger, N. Suchowerska, and D. R. McKenzie, Dosimetric consequences of gold nanoparticle clustering during photon irradiation, Med. Phys. 44, 6560-6569 (2017).

C. Hüttig and K. Stemmer, The spiral grid: A new approach to discretize the sphere and its application to mantle convection, Geochemistry, Geophysics, Geosystems 9 (2008).

E. R. Epp and H. Weiss, Experimental study of the photon energy spectrum of primary diagnostic X-rays, Phys. Med. Biol. 11, 225 (1966).

P. Oliver and R. M. Thomson, Investigating energy deposition within cell populations using Monte Carlo simulations, Physics in Medicine \& Biology 63, 155018 (2018).

G. Vona et al., Isolation by size of epithelial tumor cells: a new method for the immunomorphological and molecular characterization of circulating tumor cells, The American journal of pathology 156, 57-63 (2000).

B. D. Chithrani, A. A. Ghazani, and W. C. Chan, Determining the size and shape dependence of gold nanoparticle uptake into mammalian cells, Nano letters 6, 662-668 (2006).

P. Oliver and R. M. Thomson, Cavity theory applications for kilovoltage cellular dosimetry, Phys. Med. Biol. 62, 4440 (2017).

D. B. Chithrani, S. Jelveh, F. Jalali, M. van Prooijen, C. Allen, R. G. Bristow, R. P. Hill, and D. A. Jaffray, Gold nanoparticles as radiation sensitizers in cancer therapy, Radiat. Res. 173, 719 - 728 (2010).

F. Verhaegen and I. A. Castellano, Microdosimetric characterisation of $28 \mathrm{kVp}$ MoMo RhRh RhAl WRh and MoRh mammography x-ray spectra, Radiat. Prot. Dosim. 99, $393-396$ (2002).

P. Oliver and R. M. Thomson, A Monte Carlo Study of Macroscopic and Microscopic Dose Descriptors for Kilovoltage Cellular Dosimetry, Med. Phys. 42, 3674 (abs) (2015).

P. A. Oliver and R. M. Thomson, Investigating energy deposition in glandular tissues for mammography using multiscale Monte Carlo simulations, Med. Phys. 46, 1426-1436 (2019).

S. Seltzer, J. Fernández-Varea, P. Andreo, P. Bergstrom, D. Burns, I. K. Bronić, C. Ross, and F. Salvat, Key data for ionizing-radiation dosimetry: measurement standards and applications, ICRU Report 90, (2016). 
123 E. Weinan, B. Engquist, X. Li, W. Ren, and E. Vanden-Eijnden, Heterogeneous multiscale methods: a review, Commun. Comput. Phys 2, 367-450 (2007).

124 W. Ren and E. Weinan, Heterogeneous multiscale method for the modeling of complex fluids and micro-fluidics, J. of Comput. Phys. 204, 1-26 (2005).

125 K. Matouš, M. G. Kulkarni, and P. H. Geubelle, Multiscale cohesive failure modeling of heterogeneous adhesives, J. of the Mech. and Phys. of Solids 56, 1511-1533 (2008).

126 S. H. Brodersen, U. Grønbjerg, B. Hvolbæk, and J. Schiøtz, Understanding the catalytic activity of gold nanoparticles through multi-scale simulations, Journal of catalysis $\mathbf{2 8 4}$, 34-41 (2011). 